Supporting Information

for

\title{
Insulin analogs with altered insulin receptor isoform binding specificities and enhanced aggregation stabilities
}

Terezie Páníková, Katarína Mitrová, Tereza Halamová, Karolína Mrzílková, Jan Pícha, Martina Chrudinová, Andrii Kurochka, Irena Selicharová, Lenka Žáková, and Jiř̌i Jiráček*

Institute of Organic Chemistry and Biochemistry, Czech Academy of Sciences, Flemingovo n. 2, 11610 Prague 6, Czech Republic.*Corresponding author: jiracek@uochb.cas.cz

\section{Table of Contents:}

Page S2:

Table S1 shows sequence alignment of all analogs prepared in the study

Pages S3-S9:

Synthesis of precursors for [des(B27-B30), $\mathrm{pTyr}^{\mathrm{B} 26}$, amide $\left.{ }^{\mathrm{B} 26}\right]$-insulin (analog 48)

Schemes S1-S2

Figure S1 shows analytical HPLC of precursor 8a

Pages S10-S58:

Physical-chemical characterization of insulin analogs 1-48

Figures S2-S49 show analytical HPLC chromatograms and mass spectra of analogs 1-48.

Pages S59-S66:

Receptor binding affinities ( $K_{\mathrm{d}}$ values) of analogs 1-48 for IR-A and IR-B

Tables S2-S13

Page S67:

Representative binding curves of analog 14 and human insulin on IR-A and IR-B

Figures S50 and S51

Page S68:

The abilities of analogs 14, 30 and human insulin to induce autophosphorylation of IR-A and IR-B receptors

Figure S52 
Table S1. The sequence alignment of all analogs prepared in the study. The changes in respect to human insulin are in blue. The positions that do not exist in human insulin are in italics. Amino acids are shown in single-letter codes. ${ }^{\mathrm{d}} \mathrm{E}$ is D-glutamic acid, ${ }^{\mathrm{E}} \mathrm{E}$ is homo-glutamic acid, ${ }^{\mathrm{g}} \mathrm{E}$ is $\gamma$-carboxyglutamic acid and ${ }^{\mathrm{P}} \mathrm{Y}$ is a peptoid variant of tyrosine.

\begin{tabular}{|c|c|c|}
\hline Analog & A-chain & B-chain \\
\hline $\begin{array}{c}\text { Human insulin } \\
\text { (A1-A21 and B1-B30) }\end{array}$ & GIVEQCCTSICSLYQLENYCN & FVNQHLCGSHLVEALYLVCGERGFFYTPKT \\
\hline 1 & GIVEQCCTSICSLYQLENYCN & FVNQHLCGSHLVEALYLVCGERGFFYDTSS \\
\hline 2 & GIVEQCCTSICSLYQLENYCN & FVNQHLCGSHLVEALYLVCGERGFFYETSS \\
\hline 3 & GIVEQCCTSICSLYQLENYCN & FVNQHLCGSHLVEALYLVCGERGFFYTETSS \\
\hline 4 & GIVEQCCTSICSLYQLENYCN & FVNQHLCGSHLVEALYLVCGERGFFYTPKTDTSS \\
\hline 5 & GIVEQCCTSICSLYQLENYCN & FVNQHLCGSHLVEALYLVCGERGFFNTPKTDTSS \\
\hline 6 & GIVEQCCTSICSLYQLENYCN & FVNQHLCGSHLVEALYLVCGERGFFYTPKT-amide \\
\hline 7 & GIVEQCCTSICSLYQLENYCN & FVNQHLCGSHLVEALYLVCGERGFFYTPKTG-amide \\
\hline 8 & GIVEQCCTSICSLYQLENYCN & FVNQHLCGSHLVEALYLVCGERGFFYTPKTGG-amide \\
\hline 9 & GIVEQCCTSICSLYQLENYCN & FVNOHLCGSHLVEALYLVCGERGFFYTPKTGGG-amide \\
\hline 10 & GIVEQCCTSICSLYQLENYCN & FVNQHLCGSHLVEALYLVCGERGFFYTPKTE-amide \\
\hline 11 & GIVEQCCTSICSLYQLENYCN & FVNQHLCGSHLVEALYLVCGERGFFYTKPTG-amide \\
\hline 12 & GIVEQCCTSICSLYQLENYCN & FVNQHLCGSHLVEALYLVCGERGFFYTPATG-amide \\
\hline 13 & GIVEQCCTSICSLYQLENYCN & FVNQHLCGSHLVEALYLVCGERGFFYTPATD-amide \\
\hline 14 & GIVEQCCTSICSLYQLENYCN & FVNQHLCGSHLVEALYLVCGERGFFYTPATE-amide \\
\hline 15 & GIVEQCCTSICSLYQLENYCN & FVNQHLCGSHLVEALYLVCGERGFFYTPATE \\
\hline 16 & GIVEQCCTSICSLYQLENYCN & FVNQHLCGSHLVEALYLVCGERGFFYTKPTE \\
\hline 17 & GIVEQCCTSICSLYQLENYCN & FVNQHLCGSHLVEALYLVCGERGFFYTPKTE \\
\hline 18 & GIVEQCCTSICSLYQLENYCN & FVNQHLCGSHLVEALYLVCGERGFFYTPATQ-amide \\
\hline 19 & GIVEQCCTSICSLYQLENYCN & FVNQHLCGSHLVEALYLVCGERGFFYTPATH-amide \\
\hline 20 & GIVEQCCTSICSLYQLENYCN & FVNQHLCGSHLVEALYLVCGERGFFYTPATA-amide \\
\hline 21 & GIVEQCCTSICSLYQLENYCN & FVNQHLCGSHLVEALYLVCGERGFFYTPATT-amide \\
\hline 22 & GIVEQCCTSICSLYQLENYCN & FVNQHLCGSHLVEALYLVCGERGFFNTPATY-amide \\
\hline 23 & GIVEQCCTSICSLYQLENYCN & FVNQHLCGSHLVEALYLVCGERGFFNTPATM-amide \\
\hline 24 & GIVEQCCTSICSLYQLENYCN & FVNQHLCGSHLVEALYLVCGERGFFNTPAT/-amide \\
\hline 25 & GIVEQCCTSICSLYQLENYCN & FVNQHLCGSHLVEALYLVCGERGFFNTPATW-amide \\
\hline 26 & GIVEQCCTSICSLYQLENYCN & FVNQHLCGSHLVEALYLVCGERGFFNTPATP-amide \\
\hline 27 & GIVEQCCTSICSLYQLENYCN & FVNQHLCGSHLVEALYLVCGERGFFNTPAT ${ }^{d}$ E-amide \\
\hline 28 & GIVEQCCTSICSLYQLENYCN & FVNQHLCGSHLVEALYLVCGERGFFNTPAT ${ }^{h}$ E-amide \\
\hline 29 & GIVEQCCTSICSLYQLENYCN & FVNQHLCGSHLVEALYLVCGERGFFNTPAT ${ }^{g} E$-amide \\
\hline 30 & GIVEQCCTSICSLYQLEEYCN & FVNQHLCGSHLVEALYLVCGERGFFYTPKT \\
\hline 31 & GIVEQCCTSICSLEQLENYCN & FVNQHLCGSHLVEALYLVCGERGFFYTPKT \\
\hline 32 & GGIVEQCCTSICSLYQLENYCN & FVNQHLCGSHLVEALYLVCGERGFFYTPKT \\
\hline 33 & GGGIVEQCCTSICSLYQLENYCN & FVNQHLCGSHLVEALYLVCGERGFFYTPKT \\
\hline 34 & GGGGIVEQCCTSICSLYQLENYCN & FVNQHLCGSHLVEALYLVCGERGFFYTPKT \\
\hline 35 & GIVEQCCTSICSLYQLENYCN & FVNQHLCGSHLVEALYLVCGERGFFY \\
\hline 36 & GIVEQCCTSICSLYQLENYCN & FVNQHLCGSHLVEALYLVCGERGFFG \\
\hline 37 & GIVEQCCTSICSLYQLENYCN & FVNQHLCGSHLVEALYLVCGERGFFS \\
\hline 38 & GIVEQCCTSICSLYQLENYCN & FVNQHLCGSHLVEALYLVCGERGFFT \\
\hline 39 & GIVEQCCTSICSLYQLENYCN & FVNQHLCGSHLVEALYLVCGERGFFE \\
\hline 40 & GIVEQCCTSICSLYQLENYCN & FVNQHLCGSHLVEALYLVCGERGFFK \\
\hline 41 & GIVEQCCTSICSLYQLENYCN & FVNQHLCGSHLVEALYLVCGERGFFV \\
\hline 42 & GIVEQCCTSICSLYQLENYCN & FVNQHLCGSHLVEALYLVCGERGFFF \\
\hline 43 & GIVEQCCTSICSLYQLENYCN & FVNQHLCGSHLVEALYLVCGERGFFH \\
\hline 44 & GIVEQCCTSICSLYQLENYCN & FVNQHLCGSHLVEALYLVCGERGFFP \\
\hline 45 & GIVEQCCTSICSLYQLENYCN & FVNQHLCGSHLVEALYLVCGERGFFY-amide \\
\hline 46 & GIVEQCCTSICSLYQLENYCN & FVNQHLCGSHLVEALYLVCGERGFFF-amide \\
\hline 47 & GIVEQCCTSICSLYQLENYCN & FVNQHLCGSHLVEALYLVCGERGFFP-amide \\
\hline 48 & GIVEQCCTSICSLYQLENYCN & FVNQHLCGSHLVEALYLVCGERGFFPY-amide \\
\hline
\end{tabular}




\section{Synthesis of precursors for [des(B27-B30), pTyr ${ }^{\mathrm{B} 26}$, amide ${ }^{\mathrm{B} 26}$ ]-insulin (analog 48)}

Reagent and solvents (Fluorochem, Ltd.) used for the preparation of precursors of $\mathbf{5 3}$ were of an analytical grade. TLC on silica gel coated aluminum plates (Fluka) was performed in the following systems (v/v): ethyl acetate - 3oluene 1: 1 (S1), ethyl acetate - toluene $10: 90$ (S2). The compounds were visualized by exposure to UV light at $254 \mathrm{~nm}$. Flash chromatography was performed on silica gel (40-63 $\mu \mathrm{m}$, Fluka). Preparative RP-HPLC was carried out at flow rate 9 $\mathrm{ml} / \mathrm{min}$ on C18 column (Macherey-Nagel, $250 \mathrm{x} 40 \mathrm{~mm}, 5 \mu \mathrm{m})$ using a gradient $\mathrm{G} 1 \mathrm{t}=\mathrm{t}=\mathrm{min}$ (10\% B), $t=30$ min (100 \% B), t = 31 min (10\% B). Analytical RP-HPLC was performed on Nucleosil 120-5 C8 column ( $250 \times 4.6 \mathrm{~mm}, 5 \mu \mathrm{m}$, Macherey-Nagel) at flow rate $1 \mathrm{ml} / \mathrm{min}$ using a gradient G2: $t=0 \min (20 \% \mathrm{~B}), \mathrm{t}=30 \mathrm{~min}(100 \% \mathrm{~B}), \mathrm{t}=31 \mathrm{~min}(20 \% \mathrm{~B})$. Solvent A was $0.1 \% \mathrm{TFA}$ and solvent B was $80 \%$ AcCN, $0.1 \%$ TFA. Eluted compounds were detected at $218 \mathrm{~nm}$. Melting points were determined on a Boetius block and are uncorrected. ${ }^{1} \mathrm{H}$ and ${ }^{13} \mathrm{C}$ NMR spectra were recorded on Bruker AVANCE-600 spectrometer $\left({ }^{1} \mathrm{H}\right.$ at $600,13 \mathrm{MHz},{ }^{13} \mathrm{C}$ at $\left.150.9 \mathrm{MHz}\right)$ in $\mathrm{CDCl}_{3}$ or $\mathrm{D}_{2} \mathrm{O}$ at $300 \mathrm{~K}$. The 2D-H,H-COSY, 2D-H, C-HSQC and 2D-H,C-HMBC spectra were recorded and used for the structural assignment of proton and carbon signals. IR spectra were recorded on Nicolet 6700 FT-IR from Thermo Scientific. HRMS spectra were recorded as specified above.

The synthetic steps leading to the insulin analog $\mathbf{4 8}$ are shown in Schemes S1 and S2.

Amine 6a, a crucial precursor for the synthesis of Gly-Phe-Phe-pTyr ${ }^{\mathrm{B} 26}-\mathrm{C} \alpha$-carboxamide $\mathbf{8 a}$ was prepared as shown in Scheme S1. Tyramine 1a was chosen as a starting compound. To avoid the potential formation of side products during the solid phase synthesis of 8a (shown in Scheme S2), we decided to protect the phenolic hydroxy group of the compound 6a by tert. Butyl moiety. Firstly, with respect to the reactivities of both functionalities in 1a, it was necessary to protect the amine. Phthalimide appeared as a suitable choice, because it can be removed from the primary amine by treatment with hydrazine in ethanol. Thus, heating 1a with phthalic anhydride afforded intermediate 2a in an excellent yield. However, the product 2a was completely insoluble in all standard solvents usually used for tert-butylating reactions (e.g. DCM, dioxane or THF). 


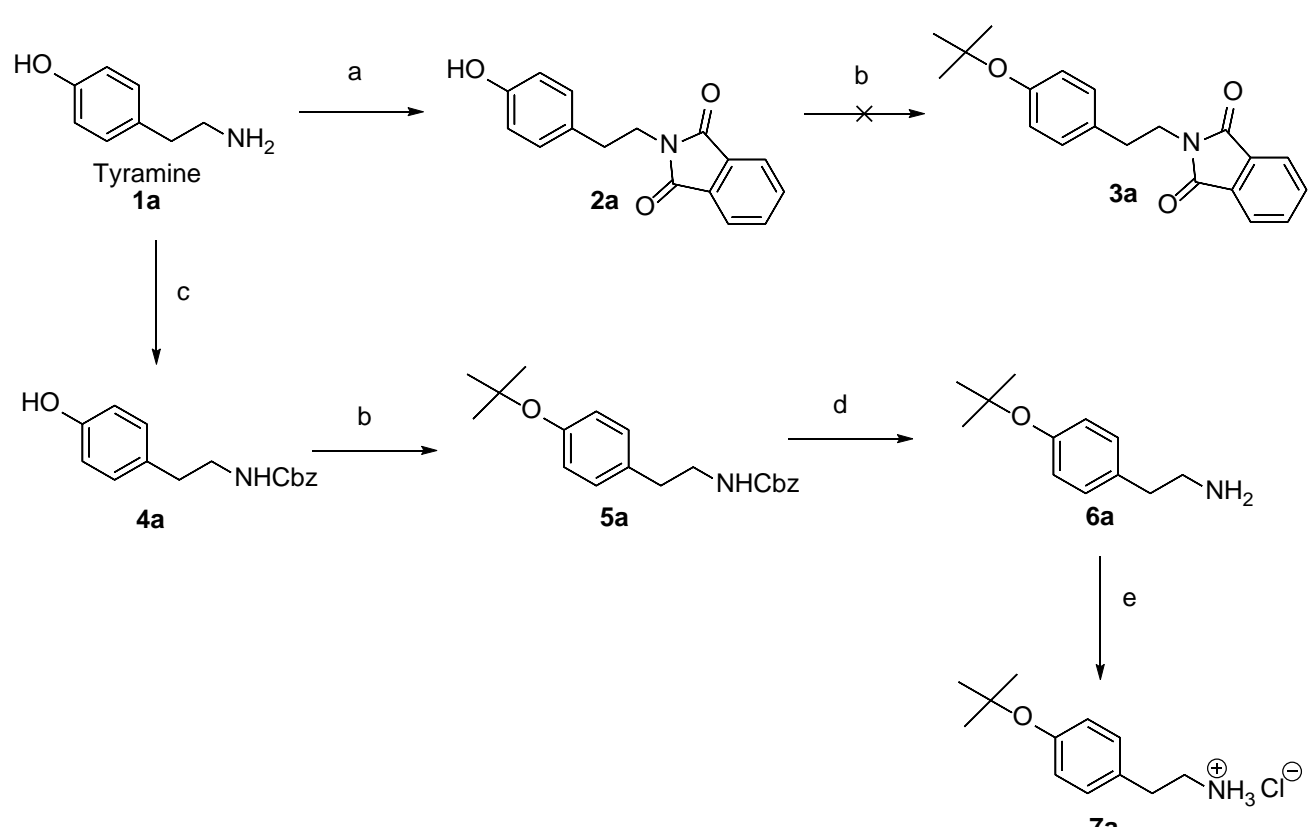

$7 a$

Scheme S1. Reagents, conditions and yields: (a) phthalic anhydride, acetic acid, toluene, heating at $110{ }^{\circ} \mathrm{C}$ for 19 hours (95\%); (b) isobutene, $\mathrm{H}_{2} \mathrm{SO}_{4}$, DCM, at rt for 4 days, (50 \% for $5 \mathbf{a}$ ); (c) Cbz$\mathrm{Cl}, \mathrm{NaHCO}_{3}$, water and dioxane, at $0{ }^{\circ} \mathrm{C}$ for 1 hour then rt overnight (83 \%); (d) $10 \% \mathrm{Pd} / \mathrm{C}, \mathrm{H}_{2}, 10$ psi, methanol at rt for 8 hours (91\%); e) $4 \mathrm{M} \mathrm{HCl} /$ dioxane, at rt for 40 minutes (82\%).

We also tested the reaction of 2a with isobutene catalyzed with conc. sulfuric acid in DCM under heterogenous conditions, but only traces of the product (3a) were observed. Based on this finding, we decided to apply a protocol published by Uno et al. (Tetrahedron Lett. 1999, 40, 1475). Different chemoselectivities of hydroxy and amino groups allowed tyramine $\mathbf{1 a}$ to react with Cbz$\mathrm{Cl}$, to afford carbamate $\mathbf{4 a}$, which was, in contrast to $\mathbf{2 a}$, readily soluble in DCM and underwent a reaction with isobutene under catalysis with sulfuric acid. Intermediate 5a, isolated with $50 \%$ yield, was treated with gaseous hydrogen to give a required crude amine $\mathbf{6 a}$, which was sufficiently pure for further use in the solid-phase synthesis. An analytical amount of free amine $\mathbf{6 a}$ was also converted to its hydrochloric salt $\mathbf{7 a}$. 


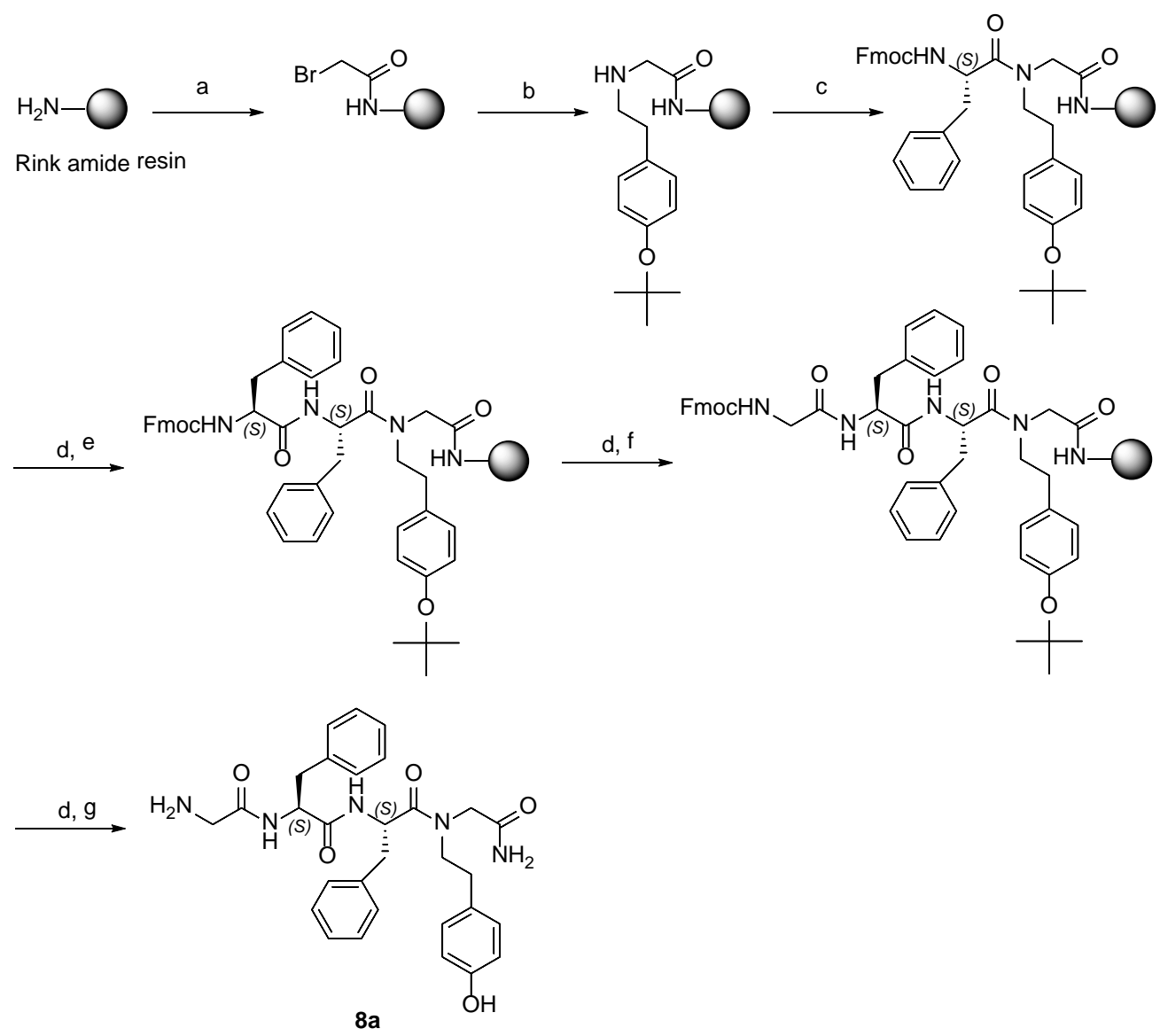

Scheme S2. Reagents and conditions: (a) bromoacetic acid, DIC, DMF, 2 x 0.5 h; (b) 6a, DMF, 2 x 1 h; (c) Fmoc-Phe, PyBroP, DIPEA, NMP, 2 x 2 hours; (d) $20 \%$ piperidine/DMF, 5 and 20 min; can Fmoc-Phe, DIC, DMF overnight then Fmoc-Phe, HBTU, DIPEA, NMP, 2 hours; (f) FmocGly, HBTU, DIPEA, NMP, 2 x 2 hours (g) TFA, TIS, $\mathrm{H}_{2} \mathrm{O}$ (95: $3: 2$ ), 1 hour at rt, (cumulative yield $57 \%)$.

\section{$N$-(4-hydroxyphenethyl)phtalimide $2 a$}

Phthalic anhydride (14.8 g; $0.1 \mathrm{~mol}$ ) and tyramine 1a (13.8 g; $0.2 \mathrm{~mol})$ were heated in a mixture of AcOH $(100 \mathrm{ml})$ and toluene $(300 \mathrm{ml})$ at $110^{\circ} \mathrm{C}$ for 19 hours. Volatile material was evaporated under a reduced pressure; the slightly brown residue was dissolved in $250 \mathrm{ml}$ of isopropyl alcohol, and a flask with the solution was placed at $5{ }^{\circ} \mathrm{C}$. After standing overnight, crystals were filtered off, washed with $250 \mathrm{ml}$ of hexane and dried under reduced pressure. Yield $25.3 \mathrm{~g}(95 \%)$. White solid, m. p. $229-231^{\circ} \mathrm{C} . \mathrm{R}_{\mathrm{f}}=0.78(\mathrm{~S} 1) .{ }^{1} \mathrm{H}$ NMR $\left(600 \mathrm{MHz}, \mathrm{DMSO} ; \mathrm{T}=25^{\circ} \mathrm{C}\right): 2.79 \mathrm{t}(2 \mathrm{H}, J=$ $\left.7.4 \mathrm{~Hz},-\mathrm{CH}_{2}-\right), 3.74 \mathrm{t}\left(2 \mathrm{H}, J=7.4 \mathrm{~Hz},-\mathrm{CH}_{2}-\mathrm{N}\right), 6.62 \mathrm{~m}(2 \mathrm{H}, 2 \mathrm{x} \mathrm{Ar}-\mathrm{H}), 6.96 \mathrm{~m}(2 \mathrm{H}, 2 \mathrm{x} \mathrm{Ar}-\mathrm{H})$, $7.825 \mathrm{~m}(4 \mathrm{H}, 4 \mathrm{x} \mathrm{Ar}-\mathrm{H}), 9.19 \mathrm{~s}(1 \mathrm{H}, \mathrm{OH}) .{ }^{13} \mathrm{C}$ NMR $\left(150.9 \mathrm{MHz}\right.$, DMSO; $\left.\mathrm{T}=25^{\circ} \mathrm{C}\right): 33.03$ $\left(-\mathrm{CH}_{2}-\right), 39.31\left(-\mathrm{CH}_{2}-\mathrm{N}\right), 115.39\left(2 \times \mathrm{Ar}=\mathrm{CH}^{-}\right), 123.19(2 \times \mathrm{Ar}=\mathrm{CH}-), 128.41(\mathrm{Ar}=\mathrm{C}<), 129.72$ $(2 \mathrm{x} \mathrm{Ar}=\mathrm{CH}-), 131.8(2 \times \mathrm{Ar}=\mathrm{C}<), 134.59\left(2 \times \mathrm{Ar}=\mathrm{CH}^{-}\right), 155.99(\mathrm{Ar}=\mathrm{C}<), 167.88(-\mathrm{CO}-\mathrm{N}-\mathrm{CO})$. IR ( $\left.\mathrm{KBr}, v_{\max } \mathrm{Cm}^{-1}\right) 3279 \mathrm{~s}, 1233 \mathrm{~s}(\mathrm{OH}) ; 1766 \mathrm{~s}, 1682$ vs (C=O), $2947 \mathrm{~m}, 2850 \mathrm{w}\left(\mathrm{CH}_{2}\right)$; 3061w, 3022 w, 1615 s, 1597 s, 1517 vs, 1436 s, 1403 vs, 1266 s, 1177 s, 1114 s, 1021 m, 947 s, 816 s, 
720 vs, 687 m, 530 s (rings). HRMS (ESI) calcd for $\mathrm{C}_{16} \mathrm{H}_{13} \mathrm{O}_{3} \mathrm{NNa}[\mathrm{M}+\mathrm{Na}]^{+}$290.07876; found: 290.07873 .

\section{$N$-(benzyloxycarbonyl)-2-(4-hydroxyphenyl)ethylamine $4 \boldsymbol{a}$}

Cbz-Cl (14.1 ml; $0.1 \mathrm{~mol})$ in $250 \mathrm{ml}$ of an ice-cooled dioxane was added dropwise to a solution of tyramine 1a (13.8 g; $0.1 \mathrm{~mol})$ and $\mathrm{NaHCO}_{3}(25.2 \mathrm{~g} ; 0.3 \mathrm{~mol})$ in $250 \mathrm{ml}$ of ice-cooled water. The reaction mixture was stirred for 1 hour at $0{ }^{\circ} \mathrm{C}$ and then at rt for overnight. The flask was cooled again on ice and $10 \%$ citric acid was carefully added until $\mathrm{pH} \sim 3$ was reached. Then, $250 \mathrm{ml}$ of water was added, and the solution was extracted with $3 \times 250 \mathrm{ml}$ of ethyl acetate. Combined organic layers were washed with $2 \times 300 \mathrm{ml}$ of brine and dried with $\mathrm{Na}_{2} \mathrm{SO}_{4}$. The filtrate was evaporated under reduced pressure to afford a colorless oil, which was triturated at $-20^{\circ} \mathrm{C}$ in a mixture of ethyl acetate- ether-petroleum ether. Yield $22.5 \mathrm{~g}(83 \%)$. White solid, m. p. $100{ }^{\circ} \mathrm{C} . \mathrm{R}_{\mathrm{f}}=0.71(\mathrm{~S} 1) .{ }^{1} \mathrm{H}$ NMR (600 MHz, DMSO; $\left.\mathrm{T}=25{ }^{\circ} \mathrm{C}\right): 2.59 \mathrm{~m}\left(2 \mathrm{H},-\mathrm{CH}_{2}-\right), 3.15 \mathrm{~m}\left(2 \mathrm{H},-\mathrm{CH}_{2}-\right), 5.00 \mathrm{~s}(2 \mathrm{H}$, $\left.-\mathrm{CH}_{2}-\mathrm{O}\right), 6.66 \mathrm{~m}(2 \mathrm{H}, 2 \mathrm{x} \mathrm{Ar}-\mathrm{H}), 6.96 \mathrm{~m}(2 \mathrm{H}, 2 \mathrm{x} \mathrm{Ar}-\mathrm{H}), 7.28-7.37 \mathrm{~m}(5 \mathrm{H}, 5 \mathrm{x} \mathrm{Ar}-\mathrm{H}), 7.29 \mathrm{t}$ $(1 \mathrm{H}, J=5.5 \mathrm{~Hz}, \mathrm{NH}), 9.165 \mathrm{~s}(1 \mathrm{H}, \mathrm{OH}) .{ }^{13} \mathrm{C}$ NMR $\left(150.9 \mathrm{MHz}, \mathrm{DMSO} ; \mathrm{T}=25{ }^{\circ} \mathrm{C}\right): 34.81$ $\left(-\mathrm{CH}_{2}-\right), 42.43\left(-\mathrm{CH}_{2}-\mathrm{N}\right), 65.24\left(2 \mathrm{H},-\mathrm{CH}_{2}-\mathrm{O}\right), 115.26\left(2 \mathrm{x} \mathrm{Ar}=\mathrm{CH}^{-}\right), 127.84(2 \mathrm{x} \mathrm{Ar}=\mathrm{CH}-)$, $127.90\left(\mathrm{Ar}=\mathrm{CH}^{-}\right), 128.55\left(2 \times \mathrm{Ar}=\mathrm{CH}^{-}\right), 129.47(\mathrm{Ar}=\mathrm{C}<), 129.68\left(2 \times \mathrm{Ar}=\mathrm{CH}^{-}\right), 137.50(\mathrm{Ar}$ $=\mathrm{C}<), 155.80(\mathrm{Ar}=\mathrm{C}<), 156.20(\mathrm{O}-\mathrm{CO}-\mathrm{N}) . \mathrm{IR}\left(\mathrm{KBr}, v_{\max } \mathrm{cm}^{-1}\right) 3328 \mathrm{~s}, 1245 \mathrm{vs}(\mathrm{OH}) ; 1686$ vs (C=O); 1545 s (amide II); 2939 m ( $\left.\mathrm{CH}_{2}\right)$; $3091 \mathrm{vw}, 3062 \mathrm{~m}, 3030$ m, 1613 m, $1602 \mathrm{~m}, 1515 \mathrm{vs,}$ 1454 s, 1110 m, 824 m, 746 m, 696 s, 528 m (rings). HRMS (ESI) calcd for $\mathrm{C}_{16} \mathrm{H}_{17} \mathrm{O}_{3} \mathrm{NNa}[\mathrm{M}+\mathrm{Na}]^{+}$ 294.11006; found: 294.11010 .

\section{N-benzyloxycarbonyl-2-(4-tert.butoxyphenyl)ethylamine $5 \boldsymbol{a}$}

Condensed isobutene (about $50 \mathrm{ml}$ ) and $1 \mathrm{ml}$ of $96 \% \mathrm{H}_{2} \mathrm{SO}_{4}$ were added to a dry ice-cooled (about $-20^{\circ} \mathrm{C}$ ) solution of $4 \mathbf{a}(22.5 \mathrm{~g}$; $0.082 \mathrm{~mol})$ in $200 \mathrm{ml}$ dichloromethane, placed in a pressureresistant bottle. The bottle was closed, removed from the cooling bath and allowed to react at rt for 4 days, with slow stirring. Then, the reactor was immersed again in a dry ice bath, the bottle was opened, and the reaction mixture was allowed to warm to $\mathrm{rt}$ in a well-ventilated fume hood. Thereafter, the reaction mixture was very slowly added to $200 \mathrm{ml}$ of $10 \%$ (aq.) $\mathrm{Na}_{2} \mathrm{CO}_{3}$ in a 2-L beaker. The organic phase was separated in a separatory funnel and the aqueous layer was extracted with $3 \times 100 \mathrm{ml}$ of DCM. Combined organic phases were washed with $2 \times 200 \mathrm{ml}$ of brine, and dried with $\mathrm{Na}_{2} \mathrm{SO}_{4}$. Evaporation of the filtrate afforded a dark brown oily crude product (27 g), which was subjected to flash chromatography on silica gel, using a linear gradient of ethyl acetate in petroleum ether. The fractions containing the product were evaporated to give a clear oil, which was triturated from a mixture of ethyl acetate-petroleum ether at $-20^{\circ} \mathrm{C}$. Yield $13.5 \mathrm{~g}(50 \%)$. White solid, m. p. $53-55^{\circ} \mathrm{C} . \mathrm{R}_{\mathrm{f}}=0.40$ (S2). ${ }^{1} \mathrm{H}$ NMR $\left(600 \mathrm{MHz}, \mathrm{DMSO} ; \mathrm{T}=25^{\circ} \mathrm{C}\right): 1.33 \mathrm{~s}(9 \mathrm{H}, 3 \mathrm{x}$ $\left.\mathrm{CH}_{3}\right), 2.77 \mathrm{t}\left(2 \mathrm{H}, J=7.0 \mathrm{~Hz},-\mathrm{CH}_{2}-\right), 3.44 \mathrm{q}\left(2 \mathrm{H}, J=7.4 \mathrm{~Hz},-\mathrm{CH}_{2}-\mathrm{N}\right), 4.80$ br t $(1 \mathrm{H}, J=7.0 \mathrm{~Hz}$, $\mathrm{NH}), 5.10 \mathrm{~s}\left(2 \mathrm{H},-\mathrm{CH}_{2}-\mathrm{O}\right), 6.92 \mathrm{~m}(2 \mathrm{H}, 2 \mathrm{x} \mathrm{Ar}-\mathrm{H}), 7.06 \mathrm{~m}(2 \mathrm{H}, 2 \mathrm{x} \mathrm{Ar}-\mathrm{H}), 7.31-7.37 \mathrm{~m}(5 \mathrm{H}, 5 \mathrm{x}$ $\mathrm{Ar}-\mathrm{H}) .{ }^{13} \mathrm{C}$ NMR $\left(150.9 \mathrm{MHz}, \mathrm{DMSO} ; \mathrm{T}=25^{\circ} \mathrm{C}\right): 28.79(3 \mathrm{x} \mathrm{CH}), 35.24\left(-\mathrm{CH}_{2}-\right), 42.23$ $\left(-\mathrm{CH}_{2}-\mathrm{N}\right), 66.59\left(-\mathrm{CH}_{2}-\mathrm{O}\right), 78.28(-\mathrm{O}-\mathrm{C} \equiv), 124.29\left(2 \times \mathrm{Ar}=\mathrm{CH}^{-}\right), 128.08(3 \times \mathrm{Ar}=\mathrm{CH}-), 128.48$ $\left(2 \times \mathrm{Ar}=\mathrm{CH}^{-}\right), 129.07\left(2 \times \mathrm{Ar}=\mathrm{CH}^{-}\right), 133.43(\mathrm{Ar}=\mathrm{C}<), 136.53(\mathrm{Ar}=\mathrm{C}<), 153.87(\mathrm{Ar}=\mathrm{C}<), 156.25$ 
(O-CO-N). IR ( $\left.\mathrm{KBr}, v_{\max } \mathrm{cm}^{-1}\right) 3416 \mathrm{~m}, 3348 \mathrm{~m}(\mathrm{NH}) ; 1722$ vs, 1706 vs (C=O); 1530 vs (amide II); 2933 m ( $\left.\mathrm{CH}_{2}\right) ; 2977$ s, 2873 , 1390 s, 1366 s (CH3); 1162 vs ((C(CH3) $)$; 1237 vs, 1054 s (ArO-C); 3090w, 3064 w, 3032 w, 1608 m, 1506 vs, 1455 s, 1414 m, 1106 m, 1016 m, 1003 m, 897 s, 698 m (rings); HRMS (ESI) calcd for $\mathrm{C}_{20} \mathrm{H}_{25} \mathrm{O}_{3} \mathrm{NNa}$ [M+Na] ${ }^{+}$350.17266; found: 350.17270 .

\section{2-(4-tert.butoxyphenyl)ethylamine $\mathbf{6 a}$}

Protected amine 4a (13.1 g; $40.1 \mathrm{mmol}$ ) was dissolved in $200 \mathrm{ml}$ of methanol and $250 \mathrm{mg}$ of $10 \% \mathrm{Pd} / \mathrm{C}$ was added. The vigorously stirred reaction mixture was treated with hydrogen (15 psi) for 8 hours. TLC analysis revealed the complete disappearance of the starting compound. The catalyst was filtered through a celite and washed with $100 \mathrm{ml}$ of methanol. The filtrate was evaporated under reduced pressure to a pale-yellow semisolid, which was used for the solid-phase synthesis protocol, without any additional purification. The compound was stable for at least several months at $-80^{\circ} \mathrm{C}$. Pale yellow semisolid. Yield $7.1 \mathrm{~g}(91 \%)$.

\section{2-(4-tert.butoxyphenyl)ethylamine hydrochloride 7a}

An analytical sample of 7a was prepared as follows. Compound 6a (2.9 g; $14.8 \mathrm{mmol})$ was stirred in $20 \mathrm{ml}$ of $4 \mathrm{M} \mathrm{HCl}$ in dioxane. After 40 minutes, a white precipitate was filtered off and washed with $20 \mathrm{ml}$ of diethyl ether. Crystallization from the mixture isopropyl alcohol-ether afforded a pure product. Yield $2.8 \mathrm{~g}(82 \%)$. White solid, m. p. $215-217^{\circ} \mathrm{C} .{ }^{1} \mathrm{H}$ NMR (600 MHz, DMSO; $\left.\mathrm{T}=25^{\circ} \mathrm{C}\right): 1.25 \mathrm{~s}(9 \mathrm{H}, 3 \mathrm{x} \mathrm{CH} 3), 2.87 \mathrm{~m}\left(2 \mathrm{H},-\mathrm{CH}_{2}-\right), 2.98 \mathrm{~m}\left(2 \mathrm{H},-\mathrm{CH}_{2}-\mathrm{N}\right), 6.92 \mathrm{~m}(2 \mathrm{H}$, 2x Ar-H), $7.06 \mathrm{~m}(2 \mathrm{H}, 2 \mathrm{x} \mathrm{Ar}-\mathrm{H}), 8.27 \mathrm{br} \mathrm{s}\left(3 \mathrm{H}, \mathrm{NH}_{3}\right) .{ }^{13} \mathrm{C}$ NMR $\left(150.9 \mathrm{MHz}\right.$, DMSO; $\left.\mathrm{T}=25^{\circ} \mathrm{C}\right)$ : $28.72\left(3 \mathrm{x} \mathrm{CH}_{3}\right), 35.38\left(-\mathrm{CH}_{2}-\right), 40.13\left(-\mathrm{CH}_{2}-\mathrm{N}\right), 77.93(-\mathrm{O}-\mathrm{C} \equiv), 124.01(2 \mathrm{x} \mathrm{Ar}=\mathrm{CH}-), 129.35$ $\left(2 \mathrm{x} \mathrm{Ar}=\mathrm{CH}^{-}\right), 132.23(\mathrm{Ar}=\mathrm{C}<), 153.89(\mathrm{Ar}=\mathrm{C}<) . \mathrm{IR}\left(\mathrm{KBr}, v_{\max } \mathrm{cm}^{-1}\right) 2973$ vs $+\mathrm{vbr}, 1506$ vs $\left(\mathrm{NH}_{3}{ }^{+}\right) ; 1475 \mathrm{~s}, 1388 \mathrm{~s}, 1366$ vs $\left(\mathrm{CH}_{3}\right) ; 1162$ vs $\left(\left(\mathrm{C}\left(\mathrm{CH}_{3}\right)_{3}\right) ; 1238\right.$ vs, $1023 \mathrm{~m}(\mathrm{Ar}-\mathrm{O}-\mathrm{C}) ; 1609 \mathrm{~s}$, 1505 vs, 1415 m, 1181 vs, 1110 s, 1015 s, 899 s (rings). HRMS (ESI) calcd for $\mathrm{C}_{12} \mathrm{H}_{20} \mathrm{ON}[\mathrm{M}]^{+}$ 194.15394; found: 194.15400 .

$G l y^{B 23}-P h e^{B 24}-P h e^{B 25}-p T y r^{B 26}-a m i d e^{B 26} 8 \boldsymbol{a}$

Tetrapeptide $\mathrm{Gly}^{\mathrm{B} 23}-\mathrm{Phe}^{\mathrm{B} 24}-\mathrm{Phe}^{\mathrm{B} 25}-\mathrm{pTyr}^{\mathrm{B} 26}$-amide ${ }^{\mathrm{B} 26}$ (8a) was prepared by the solid-phase synthesis, as described in Scheme S2 and as follows. Briefly, Rink amide resin (440 mg; $290 \mu \mathrm{mol}$, $0.66 \mathrm{mmol} / \mathrm{g}$ ) was placed in a $10 \mathrm{ml}$ PP syringe, equipped with PTFE frit, and the resin was swelled successively in DMF, DCM, MeOH, DCM and DMF (10 min each, $5 \mathrm{ml}$ of each). Removal of the Fmoc protecting group from resin was carried out by treatment with $2 \mathrm{ml}$ of $20 \%$ piperidine/DMF, (5 and 20 minutes each), followed by washing with $5 \times 5 \mathrm{ml}$ DMF. Then, a solution of bromoacetic acid ( $201 \mathrm{mg}$; $1.45 \mathrm{mmol}, 5$ eq.) in $1 \mathrm{ml}$ of DMF and DIC ( $224 \mu \mathrm{l} ; 1.45 \mathrm{mmol}, 5$ eq.) in $1 \mathrm{ml}$ DMF was added to the resin. The syringe was stirred for 0.5 hour. The resin was washed with $5 \times 5 \mathrm{ml}$ DMF. The procedure was repeated once more under the same conditions. Amine $6 \mathbf{a}$ (560 mg; 2.9 mmol, 10 eq.), dissolved in $2 \mathrm{ml}$ of DMF, was added to the resin and stirred for 1 hour. The resin was washed with $5 \times 5 \mathrm{ml}$ of DMF and the procedure was repeated once more under the same conditions. 
Attachment of the remaining sequence (Gly-Phe-Phe-) was done with 4 eq. of Fmoc-amino acid, 4 eq. of pyBrop and 6 eq. of DIPEA in DMF. Conversion after each coupling reaction was checked by a ninhydrin test. The Fmoc-protecting group was removed with $20 \%$ piperidine/DMF. After the completion of synthesis, the resin was washed with DMF and DCM ( $5 \times 5 \mathrm{ml}$ of each).

Peptide 8a was cleaved from the resin by treatment with $2850 \mu \mathrm{l}$ of TFA, $90 \mu \mathrm{l}$ of TIS and 60 $\mu \mathrm{l}$ of water for 1 hour. The reaction mixture was filtered, resin was rinsed with $2 \times 5 \mathrm{ml}$ of TFA. The combined filtrates were evaporated under reduced pressure and the residue was sonicated for 5 min with $30 \mathrm{ml}$ of cold diethyl ether. The resulting slurry was centrifuged for 15 minutes at 8700 rpm. Diethyl ether was removed by decantation. The white precipitate was dried overnight in a desiccator. The compound 8a was purified by RP-HPLC and lyophilized. The analytical chromatogram of purified $\mathbf{8 a}$ is shown in Figure S1. Yield $89.9 \mathrm{mg}(57 \%)$. White lyophilizate. Two rotamers (60 : 40). Major rotamer: ${ }^{1} \mathrm{H}$ NMR $\left(600 \mathrm{MHz}\right.$, DMSO; $\left.\mathrm{T}=25^{\circ} \mathrm{C}\right): 2.62 \mathrm{~m}$ and 2.49 $\mathrm{m}\left(-\mathrm{CH}_{2}{ }^{-}\right), 2.97 \mathrm{dd}(1 \mathrm{H}, J=13.8$ and $7.3 \mathrm{~Hz})$ and $2.71 \mathrm{dd}(1 \mathrm{H}, J=13.8$ and $9.0 \mathrm{~Hz})\left(-\mathrm{CH}_{2}{ }^{-}\right)$, $2.99 \mathrm{dd}(1 \mathrm{H}, J=13.5$ and $6.3 \mathrm{~Hz})$ and $2.82 \mathrm{dd}(1 \mathrm{H}, J=13.5 \mathrm{and} 7.8 \mathrm{~Hz})\left(-\mathrm{CH}_{2}-\right), 3.50 \mathrm{~m}$ and 3.41 $\mathrm{m}\left(2 \mathrm{H},-\mathrm{CH}_{2}-\mathrm{N}\right), 3.54 \mathrm{~m}$ and $3.30 \mathrm{~m}\left(-\mathrm{CH}_{2}-\mathrm{N}\right), 3.78 \mathrm{~s}\left(2 \mathrm{H},-\mathrm{CH}_{2}-\mathrm{N}\right), 4.64 \mathrm{ddd}(1 \mathrm{H}, J=9.0,8.5$ and $7.3 \mathrm{~Hz}, \mathrm{~N}-\mathrm{CH}<), 4.91 \mathrm{ddd}(1 \mathrm{H}, J=8.2,7.8$ and $6.3 \mathrm{~Hz}, \mathrm{~N}-\mathrm{CH}<), 6.66 \mathrm{~m}(2 \mathrm{H}, 2 \mathrm{x} \mathrm{Ar}-\mathrm{H}), 6.93$ $\mathrm{m}(2 \mathrm{H}, 2 \mathrm{x} \mathrm{Ar}-\mathrm{H}), 7.07 \mathrm{bs}, 1 \mathrm{H}$ and $7.035 \mathrm{bs}, 1 \mathrm{H}\left(\mathrm{CONH}_{2}\right), 7.17-7.27 \mathrm{~m}\left(10 \mathrm{H}, 2 \mathrm{x} \mathrm{C} \mathrm{C}_{6}\right), 7.91 \mathrm{br}$ $\mathrm{s}\left(2 \mathrm{H}, \mathrm{NH}_{2}\right), 8.50 \mathrm{~d}(1 \mathrm{H}, J=8.5 \mathrm{~Hz}, \mathrm{NH}), 8.785 \mathrm{~d}(1 \mathrm{H}, J=8.2 \mathrm{~Hz}, \mathrm{NH}), 9.25 \mathrm{~s}(-\mathrm{OH}) .{ }^{13} \mathrm{C}$ NMR $\left(150.9 \mathrm{MHz}, \mathrm{DMSO} ; \mathrm{T}=25{ }^{\circ} \mathrm{C}\right): 33.84\left(-\mathrm{CH}_{2}-\right), 37.79\left(-\mathrm{CH}_{2}-\right), 40.23\left(-\mathrm{CH}_{2}-\mathrm{N}\right), 49.14$ $\left(-\mathrm{CH}_{2}-\mathrm{N}\right), 50.27(>\mathrm{CH}-\mathrm{N}), 50.74\left(-\mathrm{CH}_{2}-\mathrm{N}\right), 53.75(>\mathrm{CH}-\mathrm{N}), 38.11\left(-\mathrm{CH}_{2}-\right), 115.40(3 \mathrm{x} \mathrm{Ar}$ $\left.=\mathrm{CH}^{-}\right), 126.56\left(\mathrm{Ar}=\mathrm{CH}^{-}\right), 126.70\left(\mathrm{Ar}=\mathrm{CH}^{-}\right), 128.29\left(2 \mathrm{x} \mathrm{Ar}=\mathrm{CH}^{-}\right), 128.48\left(2 \mathrm{x} \mathrm{Ar}=\mathrm{CH}^{-}\right)$, 128.67 $(\mathrm{Ar}=\mathrm{C}<), 129.42\left(2 \times \mathrm{Ar}=\mathrm{CH}^{-}\right), 129.52(2 \mathrm{x} \mathrm{Ar}=\mathrm{CH}-), 129.81\left(2 \mathrm{x} \mathrm{Ar}=\mathrm{CH}^{-}\right), 137.11(\mathrm{Ar}$ $=\mathrm{C}<), 137.36(\mathrm{Ar}=\mathrm{C}<), 156.03(\mathrm{Ar}=\mathrm{C}<), 165.69(\mathrm{CO}-\mathrm{NH}), 170.28\left(\mathrm{CO}-\mathrm{NH}_{2}\right), 170.47(\mathrm{CO}-\mathrm{NH})$, $171.21\left(\mathrm{CO}-\mathrm{N}<\right.$ ). Minor rotamer: ${ }^{1} \mathrm{H}$ NMR (600 MHz, DMSO; $\left.\mathrm{T}=25{ }^{\circ} \mathrm{C}\right): 2.61 \mathrm{~m}$ and $2.56 \mathrm{~m}$ $\left(-\mathrm{CH}_{2}-\right), 2.98 \mathrm{dd}(1 \mathrm{H}, J=13.8$ and $4.0 \mathrm{~Hz})$ and $2.70 \mathrm{dd}(1 \mathrm{H}, J=13.8$ and $9.2 \mathrm{~Hz})\left(-\mathrm{CH}_{2}{ }^{-}\right), 2.99$ dd $(1 \mathrm{H}, J=14.0$ and $4.3 \mathrm{~Hz})$ and $2.83 \mathrm{dd}(1 \mathrm{H}, J=14.0$ and $8.8 \mathrm{~Hz})\left(-\mathrm{CH}_{2}-\right), 3.43 \mathrm{~m}$ and $3.34 \mathrm{~m}$ $\left(-\mathrm{CH}_{2}-\mathrm{N}\right), 4.185 \mathrm{~d}, 1 \mathrm{H}$ and $3.75 \mathrm{~d}, 1 \mathrm{H}\left(\mathrm{J}=17.5 \mathrm{~Hz}\left(-\mathrm{CH}_{2}-\mathrm{N}\right), 3.50 \mathrm{~m}\right.$ and $3.41 \mathrm{~m}\left(2 \mathrm{H},-\mathrm{CH}_{2}-\mathrm{N}\right)$, $4.625 \mathrm{ddd}(1 \mathrm{H}, J=9.2,8.5$ and $4.0 \mathrm{~Hz}, \mathrm{~N}-\mathrm{CH}<), 4.645 \mathrm{ddd}(1 \mathrm{H}, J=8.8,8.2$ and $4.3 \mathrm{~Hz}, \mathrm{~N}-\mathrm{CH}<)$, $6.67 \mathrm{~m}(2 \mathrm{H}, 2 \mathrm{x} \mathrm{Ar}-\mathrm{H}), 6.98 \mathrm{~m}(2 \mathrm{H}, 2 \mathrm{x} \mathrm{Ar}-\mathrm{H}), 7.17-7.27 \mathrm{~m}\left(10 \mathrm{H}, 2 \mathrm{x} \mathrm{C}_{6} \mathrm{H}_{5}\right), 7.91 \mathrm{br} \mathrm{s}\left(2 \mathrm{H}, \mathrm{NH}_{2}\right)$, $7.53 \mathrm{bs}, 1 \mathrm{H}$ and $7.27 \mathrm{bs}, 1 \mathrm{H}\left(\mathrm{CONH}_{2}\right), 8.49 \mathrm{~d}(1 \mathrm{H}, J=8.5 \mathrm{~Hz}, \mathrm{NH}), 8.66 \mathrm{~d}(1 \mathrm{H}, J=8.2 \mathrm{~Hz}, \mathrm{NH})$, $9.215 \mathrm{~s}(-\mathrm{OH}) .{ }^{13} \mathrm{C} \mathrm{NMR}\left(150.9 \mathrm{MHz}, \mathrm{DMSO} ; \mathrm{T}=25^{\circ} \mathrm{C}\right): 32.39\left(-\mathrm{CH}_{2}-\right), 37.37\left(-\mathrm{CH}_{2}-\right), 38.06$ $\left(-\mathrm{CH}_{2}-\right), 40.20\left(-\mathrm{CH}_{2}-\mathrm{N}\right), 49.69\left(-\mathrm{CH}_{2}-\mathrm{N}\right), 50.66\left(-\mathrm{CH}_{2}-\mathrm{N}\right), 50.72(>\mathrm{CH}-\mathrm{N}), 53.70(>\mathrm{CH}-\mathrm{N})$, $126.53\left(\mathrm{Ar}=\mathrm{CH}^{-}\right), 126.61(\mathrm{Ar}=\mathrm{CH}-), 128.28(2 \mathrm{x} \mathrm{Ar}=\mathrm{CH}-), 128.32(2 \times \mathrm{Ar}=\mathrm{CH}-), 129.29(\mathrm{Ar}$ $=\mathrm{C}<), 129.47(2 \mathrm{x} \mathrm{Ar}=\mathrm{CH}-), 129.49\left(2 \mathrm{x} \mathrm{Ar}=\mathrm{CH}^{-}\right), 129.68(2 \mathrm{x} \mathrm{Ar}=\mathrm{CH}-), 137.44(\mathrm{Ar}=\mathrm{C}<)$, $138.11(\mathrm{Ar}=\mathrm{C}<), 165.63(\mathrm{CO}-\mathrm{NH}), 170.52\left(\mathrm{CO}-\mathrm{NH}\right.$ and $\left.\mathrm{CO}-\mathrm{NH}_{2}\right), 171.55(\mathrm{CO}-\mathrm{N}<), 115.40$ $(3 \mathrm{x} \mathrm{Ar}=\mathrm{CH}-), 155.89(\mathrm{Ar}=\mathrm{C}<) . \mathrm{IR}\left(\mathrm{KBr}, v_{\max } \mathrm{cm}^{-1}\right) 3294 \mathrm{~s}+\operatorname{vbr}(\mathrm{NH}+\mathrm{OH}) ; 1675 \mathrm{vs}(\mathrm{C}=\mathrm{O})$, 1639 vs (C=O), 1516 m (amide II); 1498 m, 1455 m, 747 m, 702 m (ring); HRMS (ESI) calcd for $\mathrm{C}_{30} \mathrm{H}_{35} \mathrm{O}_{5} \mathrm{~N}_{5} \mathrm{Na}[\mathrm{M}+\mathrm{Na}]^{+}$568.25304; found: 568.25256. 


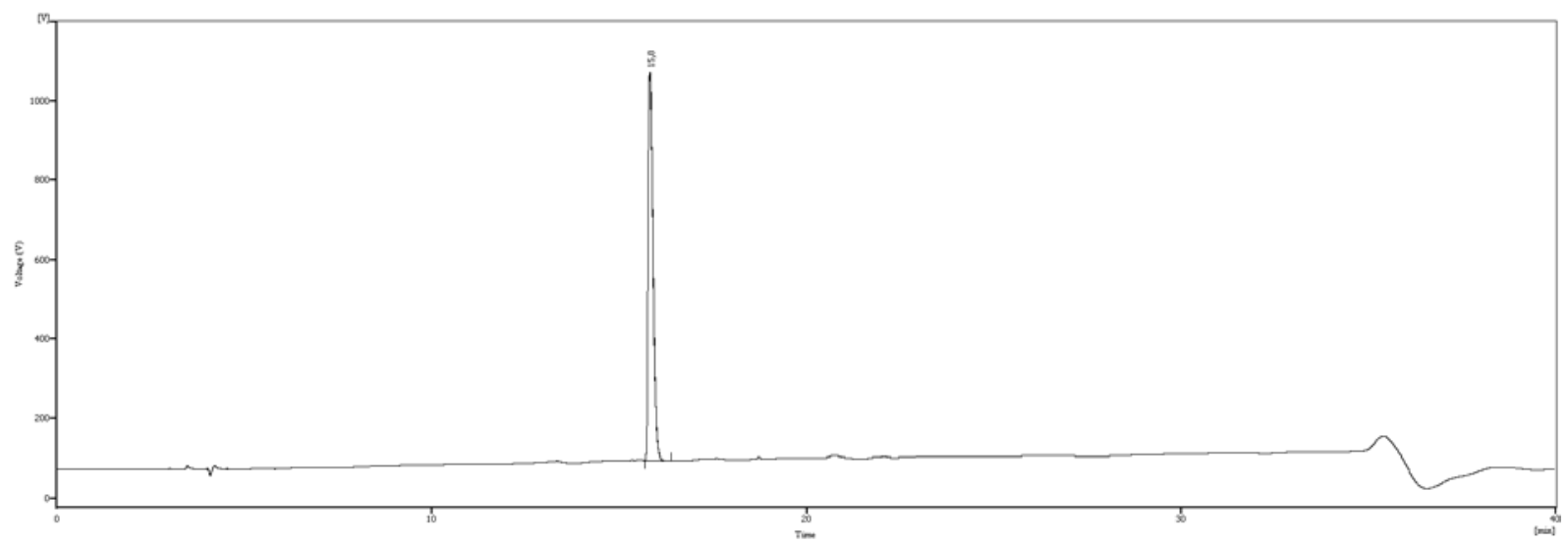

Figure S1. Analytical RP-HPLC profile of purified precursor peptide 8a using gradient G2. 


\section{Physical-chemical characterization of insulin analogs 1-48}

[Des(B23-B30)]-insulin, the precursor for the synthesis of insulin analogs, was prepared from human insulin according to Bromer et al. (Bromer, W. W.; Chance, R. E., Preparation and characterization of desoctapeptide-insulin. Biochim. Biophys. Acta 1967, 133, 219-223).

Human insulin (pharmaceutical grade, lot \# SM0H00801) was a kind gift from Dr. F. Hubálek, Novo Nordisk A/S, Maløv, Denmark.

Insulin analogs were purified on a semipreparative column VP 250/10 Nucleosil 120-5 C18 (Macherey-Nagel) and analyzed on an analytical column EC 250/4 Nucleosil 100-5 C18 HD (Macherey-Nagel) in the following gradient of $0.1 \%$ TFA (v/v) in water (solvent A) and $80 \%$ CAN, $0.1 \%$ TFA in water (solvent B) at 3 (semipreparative column) or $1 \mathrm{ml} / \mathrm{min}$ (analytical column): $\mathrm{t}=0 \min (10 \% \mathrm{~B}), \mathrm{t}=1 \mathrm{~min}(35 \% \mathrm{~B}), \mathrm{t}=21 \min (45 \% \mathrm{~B}), \mathrm{t}=35 \min (55 \% \mathrm{~B}), \mathrm{t}=36-$ $37 \min (90 \% \mathrm{~B}), \mathrm{t}=37.1 \mathrm{~min}(10 \% \mathrm{~B})$.

HRMS spectra of target compounds were obtained on the FTMS mass spectrometer LTQorbitrap XL (Thermo Fisher) in electrospray ionization mode.

Figures S2-S49 show analytical HPLC chromatograms and HR-MS spectra of analogs 1-48. 

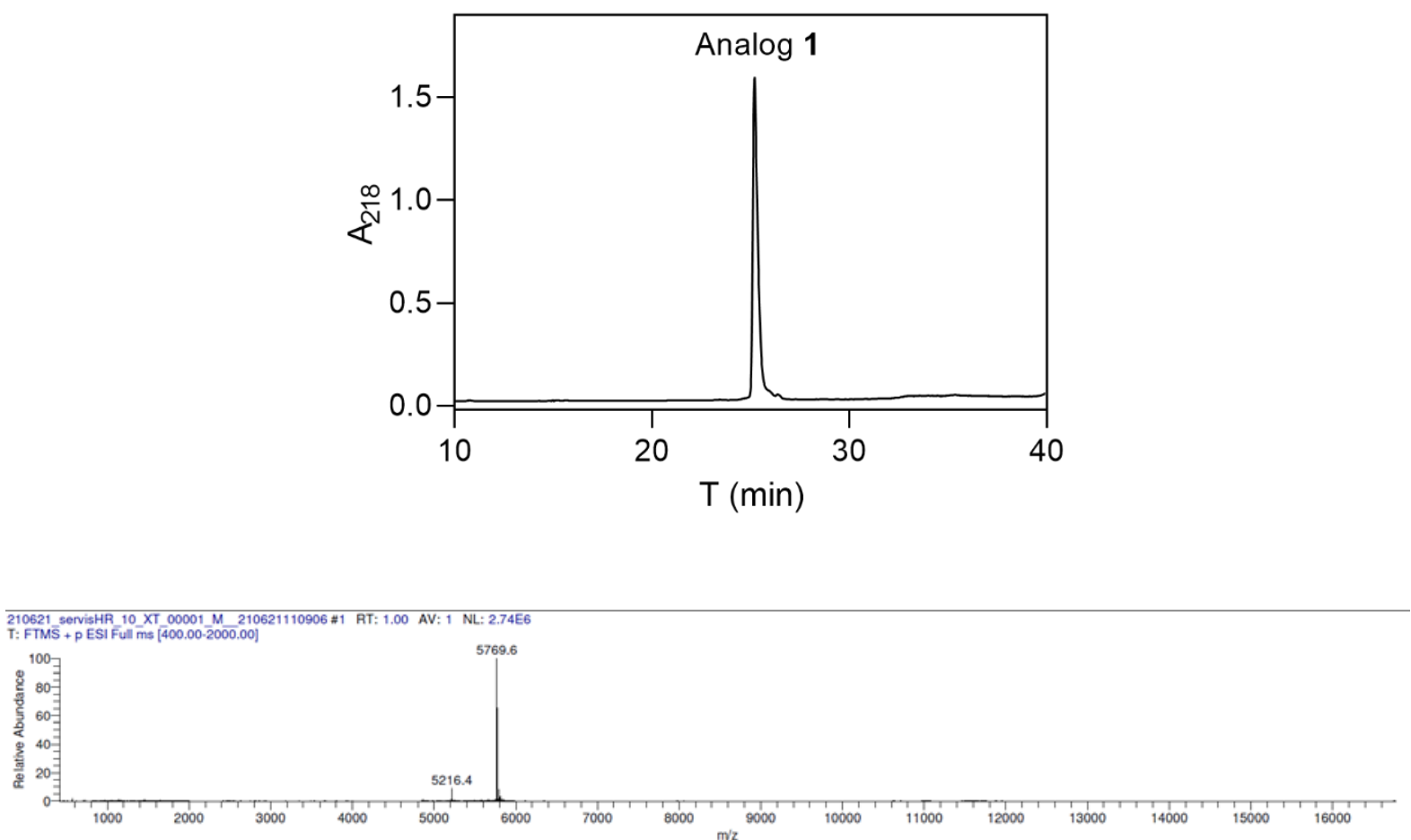

210621 servisHR 10 XT 00001 M 210621110906 \#1 RT: 1.00 AV: 1 NL: $2.74 E 6$
T: FTMS + P ESI Full ms $[400.00-2000.00]$
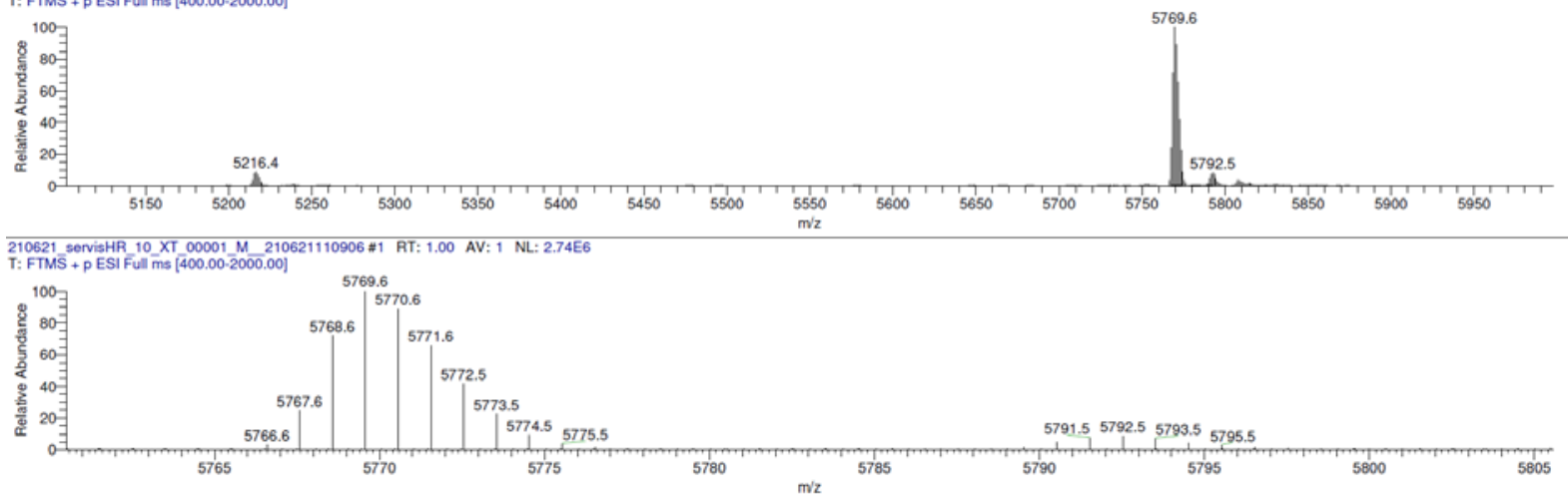

Figure S2. Upper panel: RP-HPLC analysis of analog 1 ([ $\mathrm{Asp}^{\mathrm{B} 27}$, $\mathrm{Thr}^{\mathrm{B} 28}$, $\mathrm{Ser}^{\mathrm{B} 29}$, Ser $\left.{ }^{\mathrm{B} 30}\right]$-insulin). Lower panel: HR-MS of analog 1, Mw (monoisotopic for $\mathrm{C}_{252} \mathrm{H}_{372} \mathrm{~N}_{64} \mathrm{O}_{80} \mathrm{~S}_{6}$ ) calculated 5766.5332, Mw found 5766.60. 

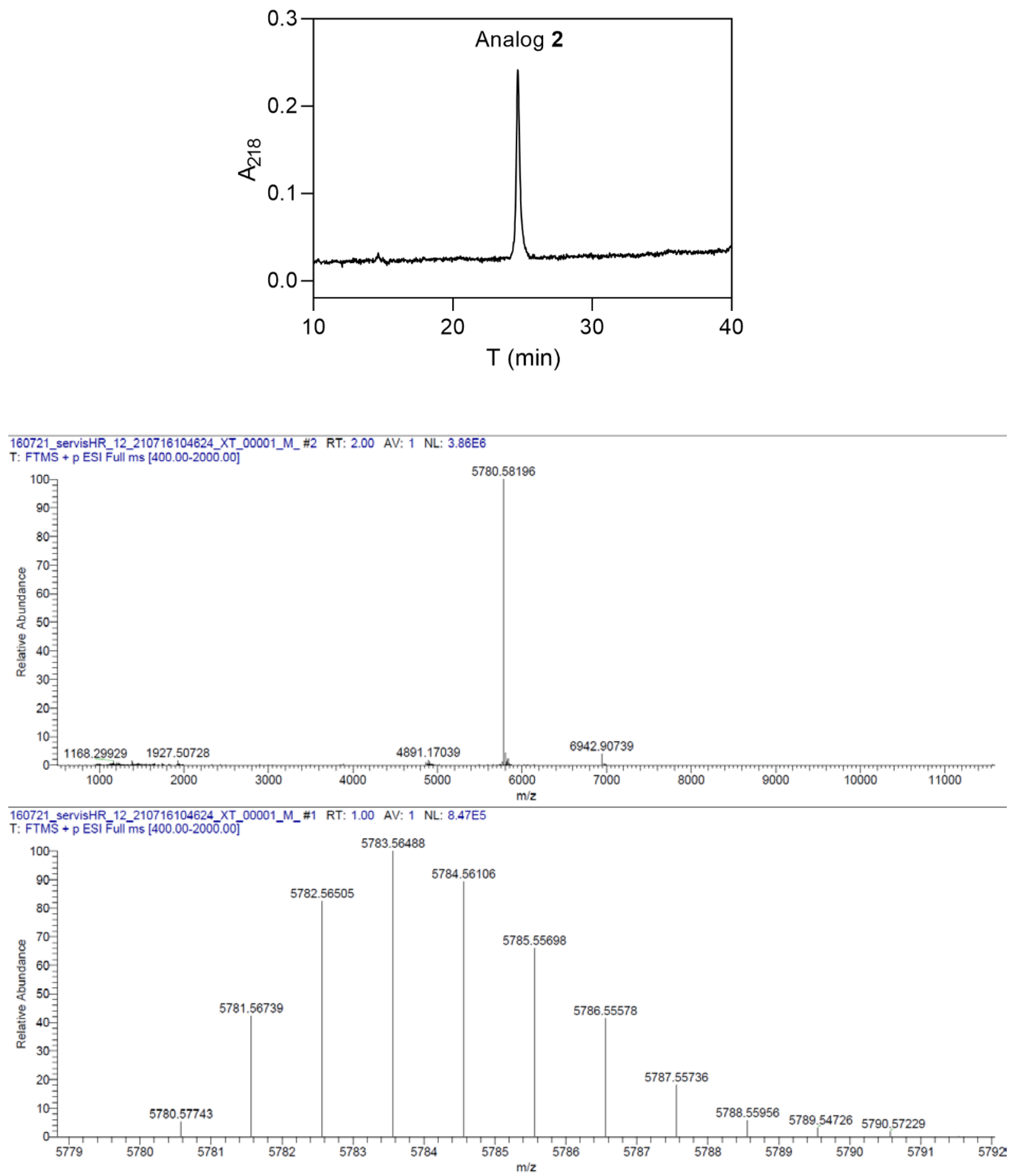

Figure S3. Upper panel: RP-HPLC analysis of analog 2 ([Glu${ }^{\mathrm{B} 27}, \mathrm{Thr}^{\mathrm{B} 28}$, $\left.\mathrm{Ser}^{\mathrm{B} 29}, \mathrm{Ser}^{\mathrm{B} 30}\right]$-insulin). Lower panel: HR-MS of analog 2, Mw (monoisotopic for $\mathrm{C}_{253} \mathrm{H}_{374} \mathrm{~N}_{64} \mathrm{O}_{80} \mathrm{~S}_{6}$ ) calculated 5780.5489, Mw 5780.5774 found. 


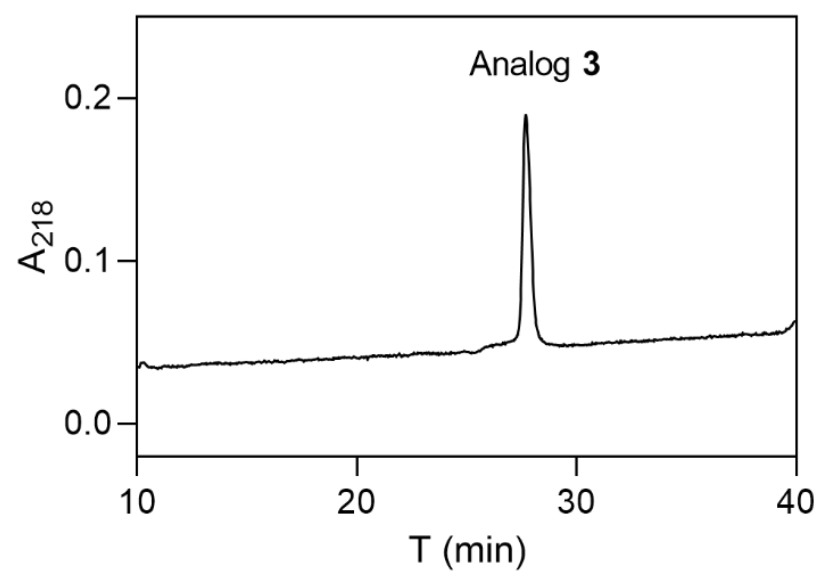

011019servisHR 2 \#67-81 RT: 1.78-2.16 AV: 15 SB: 31 0.12-0.61, 0.14-0.44 NL: 5.32E6

T: FTMS + p ESİ Full ms [200.00-2000.00]

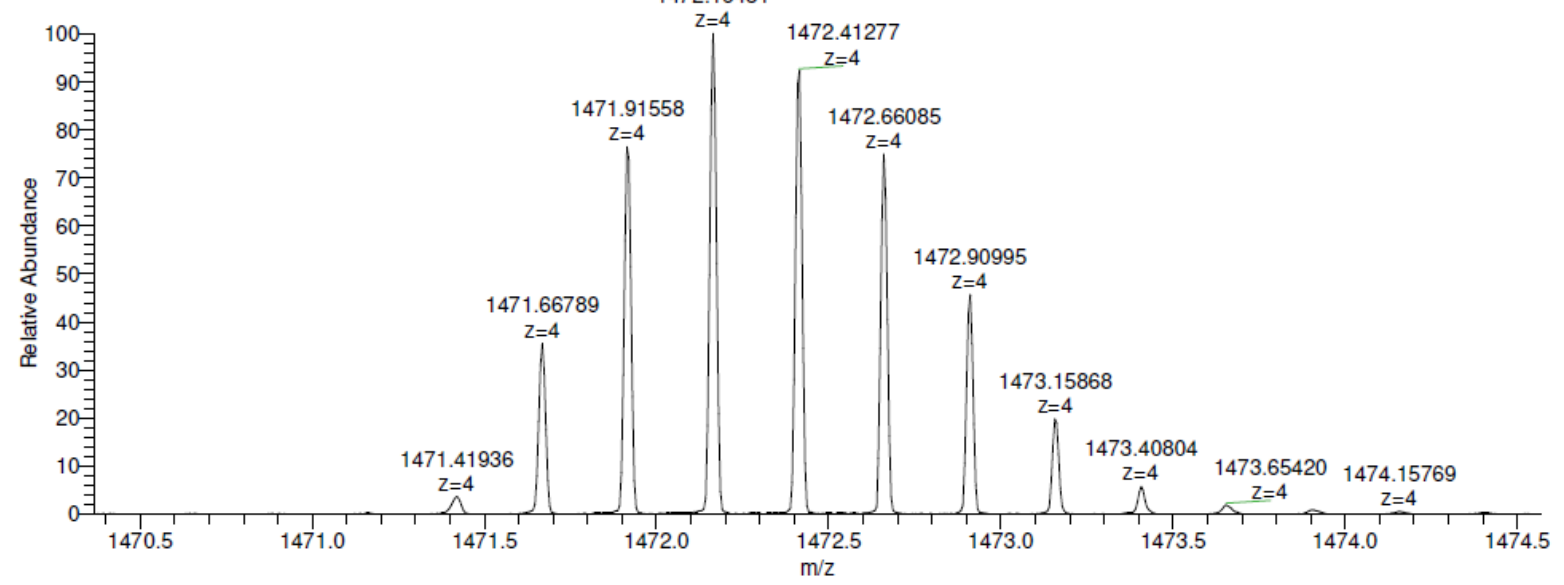

011019servisHR 2 XT_00001_M \#2 RT: 2.00 AV: 1 NL: 9.05E6

T: FTMS + p ESI Full ms [200.00-2000.00]

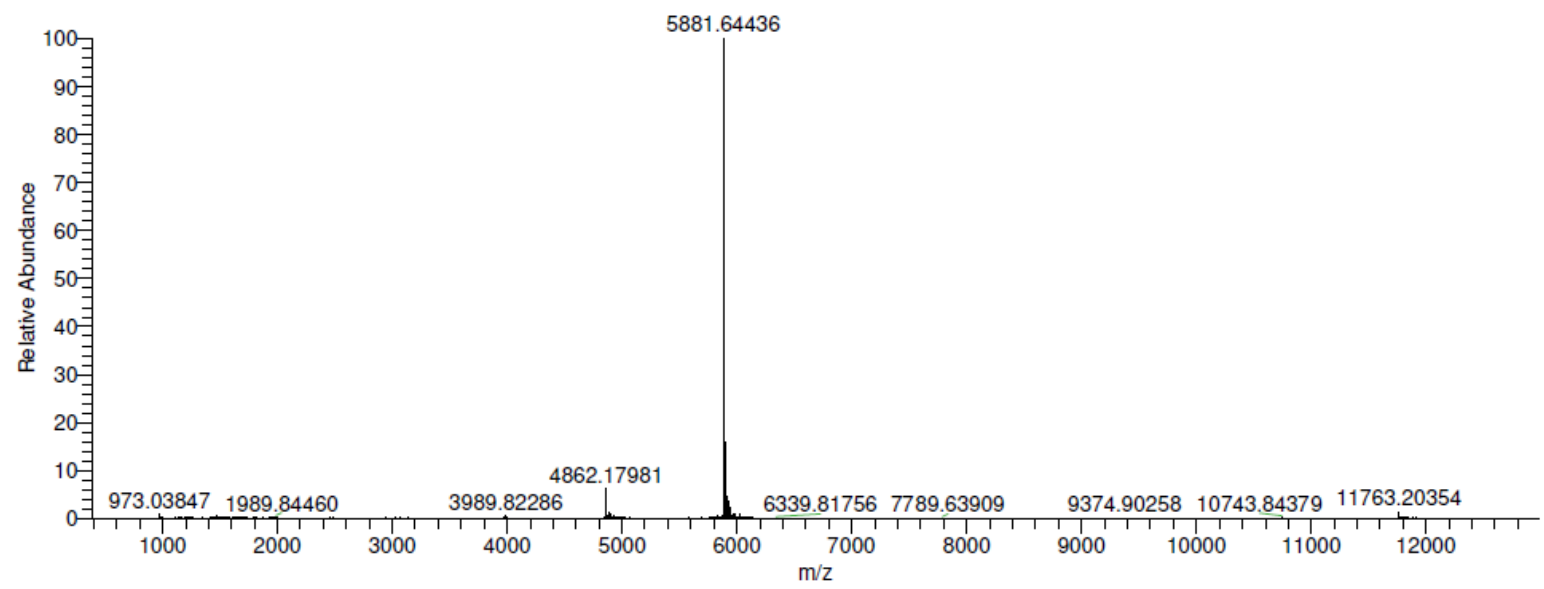

Figure S4. Upper panel: RP-HPLC analysis of analog 3 ([Glu $\left.{ }^{\mathrm{B} 28}, \mathrm{Thr}^{\mathrm{B} 29}, \mathrm{Ser}^{\mathrm{B} 30}, \mathrm{Ser}^{\mathrm{B} 31}\right]$-insulin). Lower panel: HR-MS of analog 3, Mw (monoisotopic for $\mathrm{C}_{257} \mathrm{H}_{381} \mathrm{~N}_{65} \mathrm{O}_{82} \mathrm{~S}_{6}$ ) calculated 5881.5966, Mw found 5881.6444. 

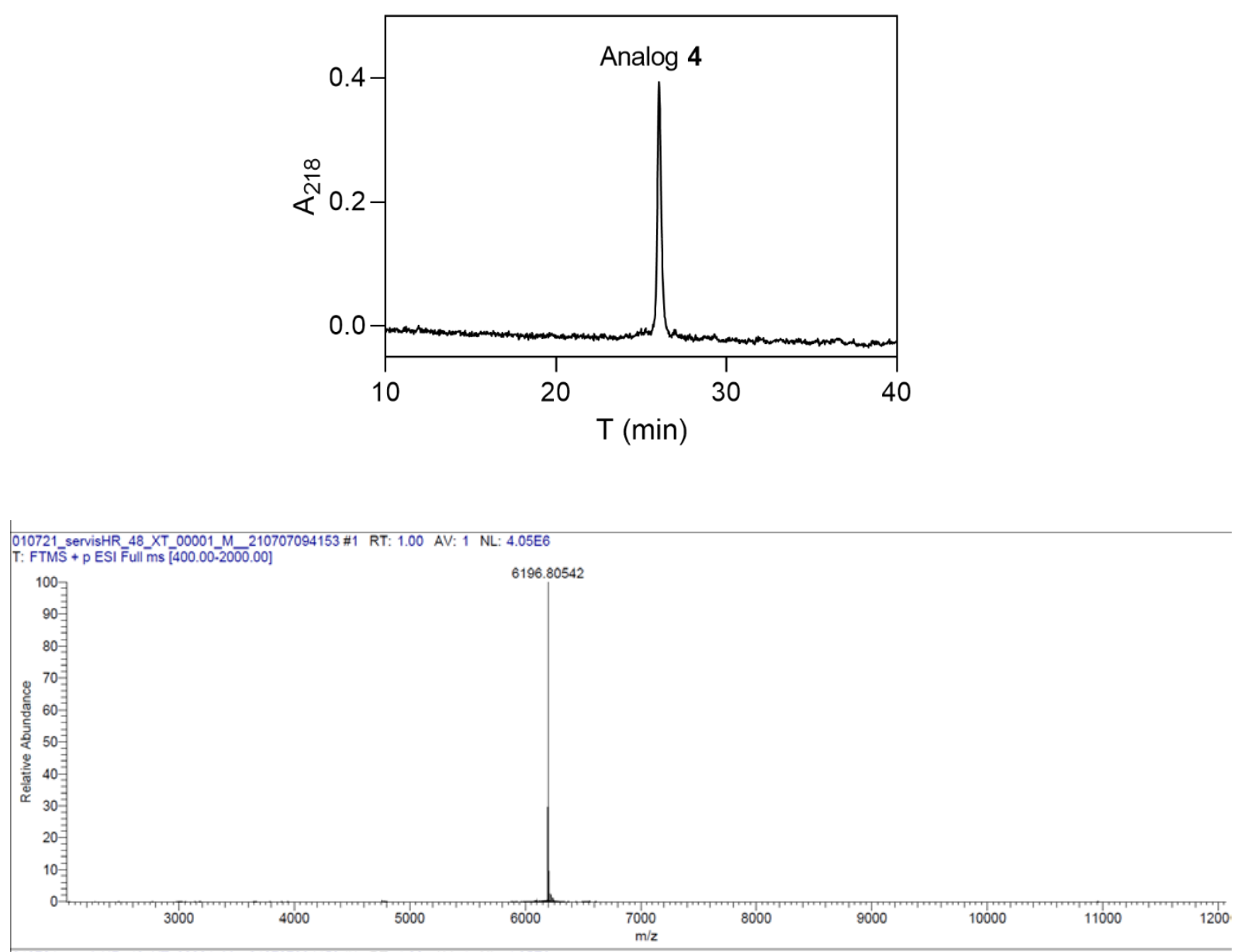

010721 servisHR_48_XT_00001_M_210707094153 \#1 RT: 1.00 AV: 1 NL: $4.05 E 6$

T: FTM $\bar{S}+p$ ESI Full $\overline{m s}[\overline{4} 00.00 \cdot 200 \overline{00} .00]$
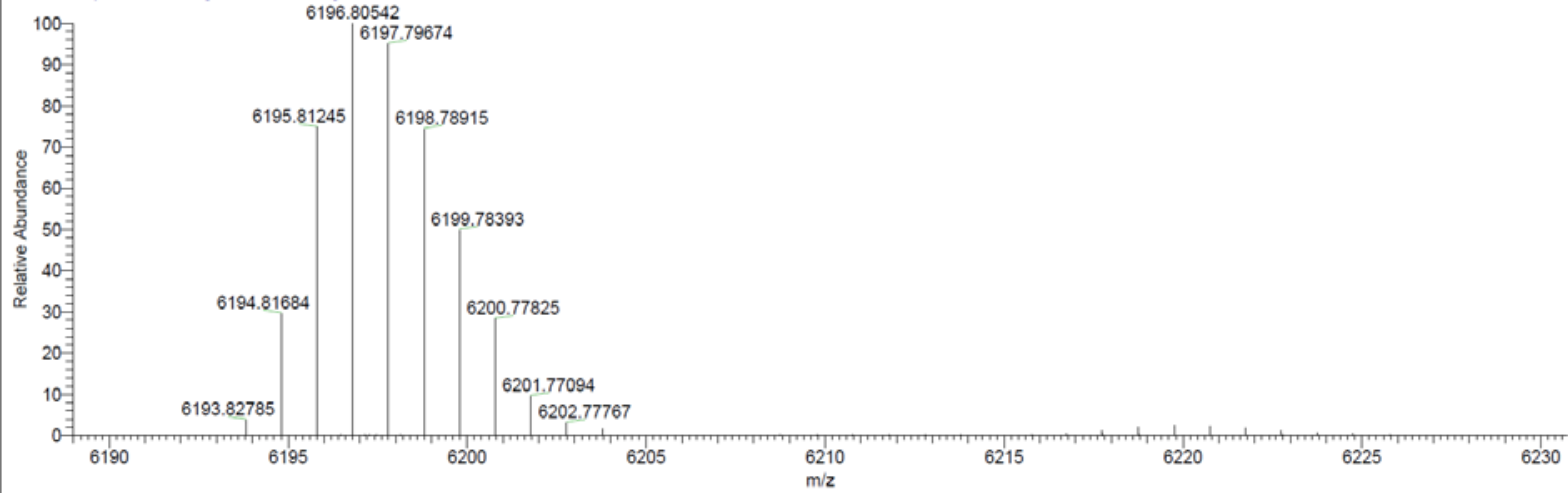

Figure S5. Upper panel: RP-HPLC analysis of analog 4 ([ $\mathrm{Asp}^{\mathrm{B} 31}$, $\mathrm{Thr}^{\mathrm{B} 32}$, $\left.\mathrm{Ser}^{\mathrm{B} 33}, \mathrm{Ser}^{\mathrm{B} 34}\right]$-insulin). Lower panel: HR-MS of analog 4, Mw (monoisotopic for $\mathrm{C}_{271} \mathrm{H}_{405} \mathrm{~N}_{69} \mathrm{O}_{86} \mathrm{~S}_{6}$ ) calculated 6193.7763, Mw found 6193.8279. 

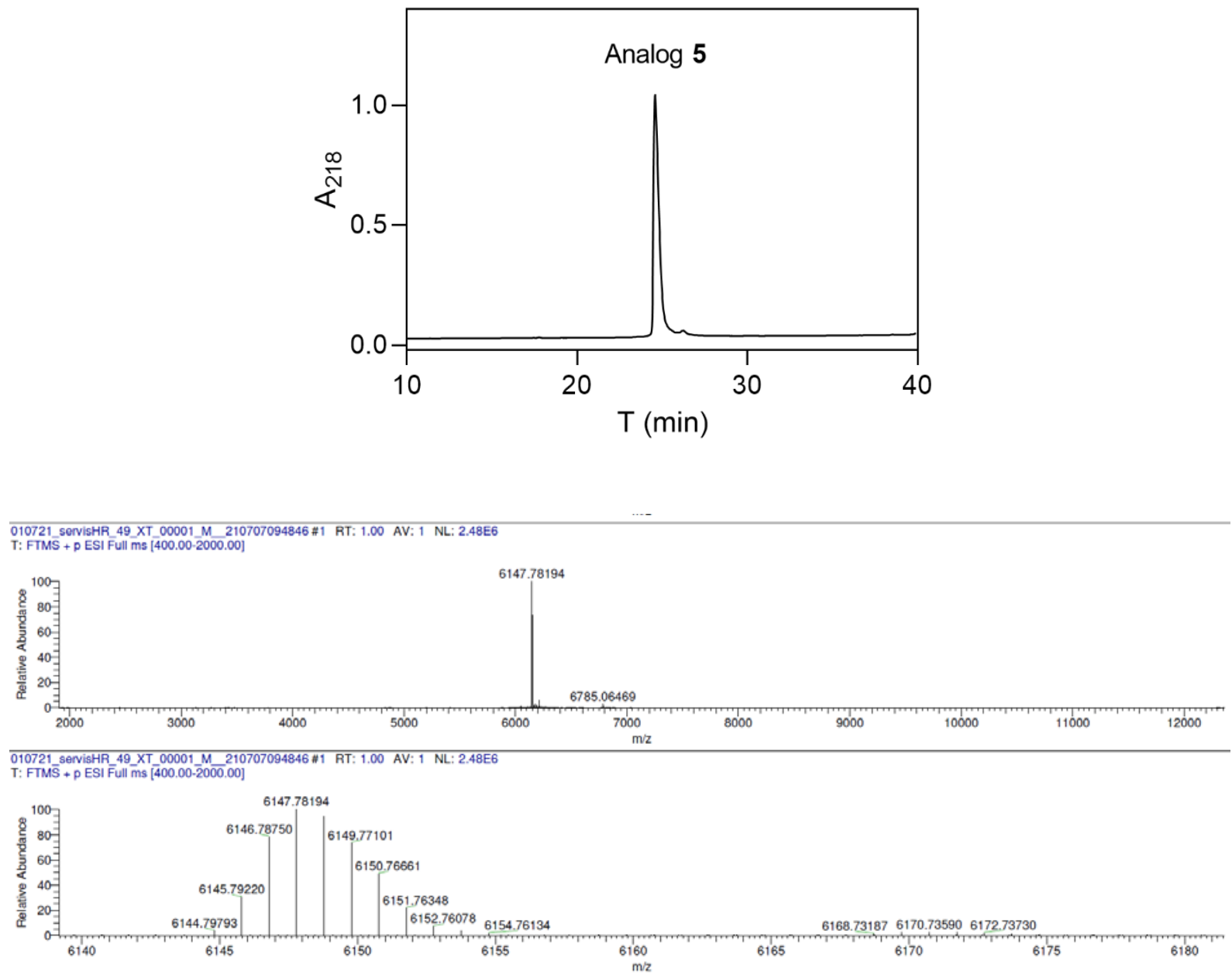

Figure S6. Upper panel: RP-HPLC analysis of analog 5 ([Asn ${ }^{\mathrm{B} 26}, \mathrm{Asp}^{\mathrm{B} 31}$, $\mathrm{Thr}^{\mathrm{B} 32}$, Ser $\left.{ }^{\mathrm{B} 33}, \mathrm{Ser}^{\mathrm{B} 34}\right]$ insulin). Lower panel: HR-MS of analog 5, Mw (monoisotopic for $\mathrm{C}_{266} \mathrm{H}_{402} \mathrm{~N}_{70} \mathrm{O}_{86} \mathrm{~S}_{6}$ ) calculated 6144.7559, Mw found 6144.7979. 

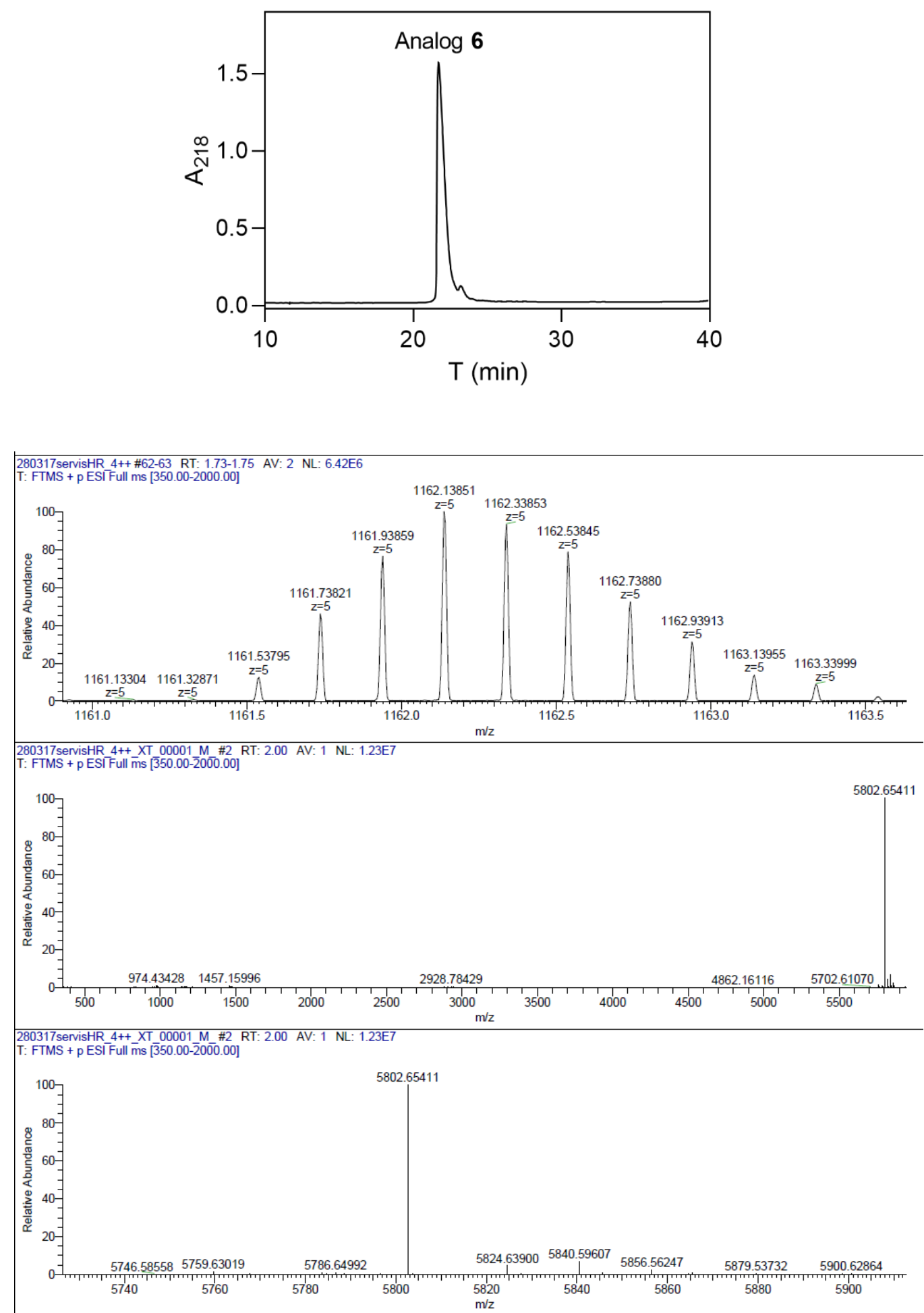

Figure S7. Upper panel: RP-HPLC analysis of analog 6 ([amide $\left.{ }^{\mathrm{B} 30}\right]$-insulin). Lower panel: HRMS of analog 6, Mw (monoisotopic for $\mathrm{C}_{257} \mathrm{H}_{384} \mathrm{~N}_{66} \mathrm{O}_{76} \mathrm{~S}_{6}$ ) calculated 5802.6536, Mw found 5802.6541. 

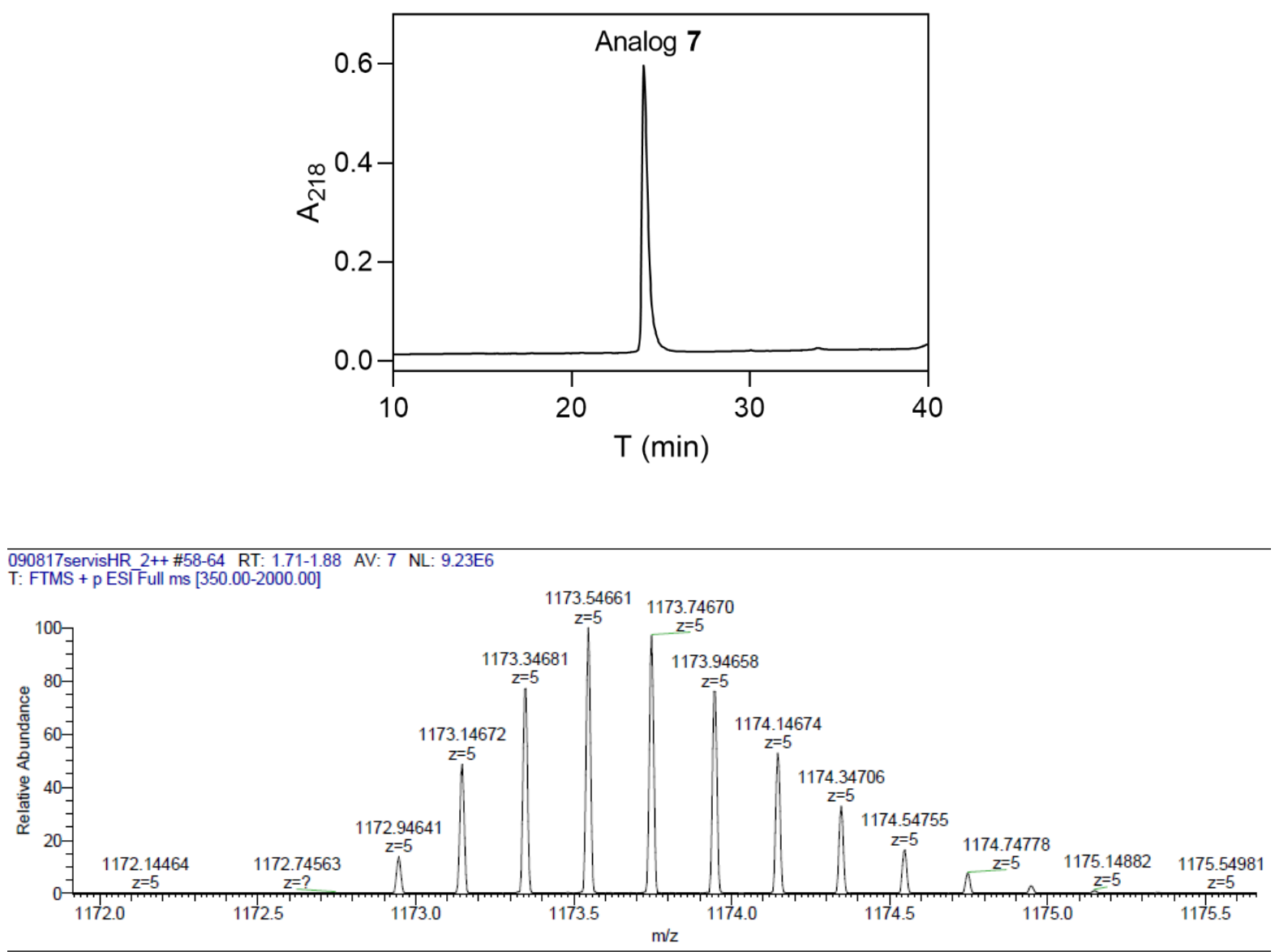

090817servisHR_2++_XT 00001_M_\#2 RT: 2.00 AV: 1 NL: $2.20 \mathrm{E} 7$

T: FTMS + p ESI Full $\mathrm{ms}[\overline{3} 50.00-\overline{2} 0 \overline{00} .00]$

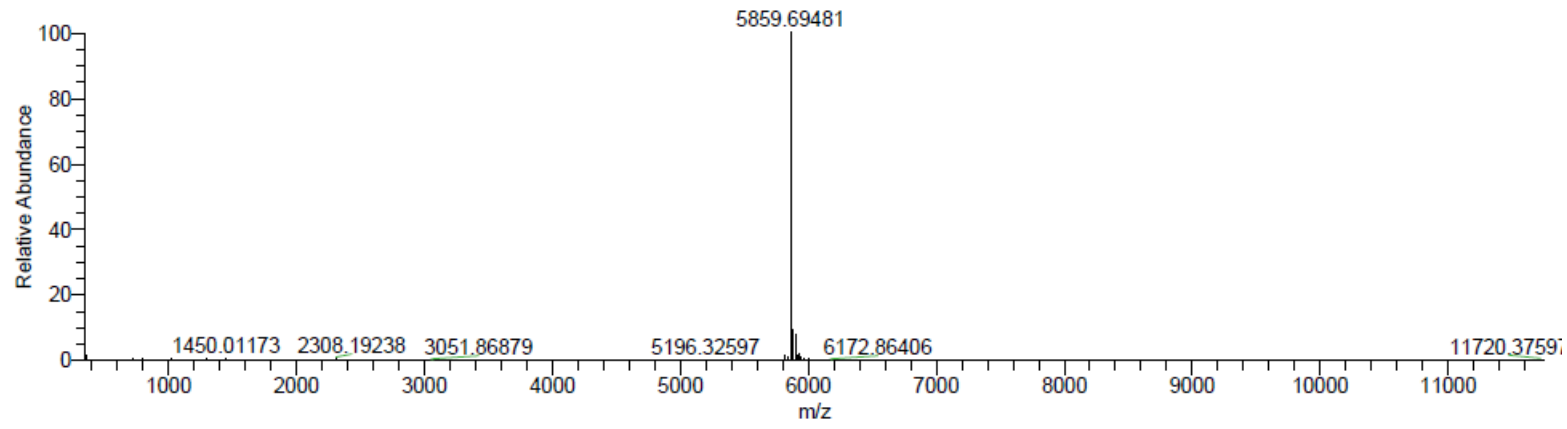

Figure S8. Upper panel: RP-HPLC analysis of analog 7 ([Gly ${ }^{\mathrm{B} 31}$, amide $\left.{ }^{\mathrm{B} 31}\right]$-insulin). Lower panel: HR-MS of analog 7, Mw (monoisotopic for $\mathrm{C}_{259} \mathrm{H}_{387} \mathrm{~N}_{67} \mathrm{O}_{77} \mathrm{~S}_{6}$ ) calculated 5859.6751, Mw found 5859.6948. 

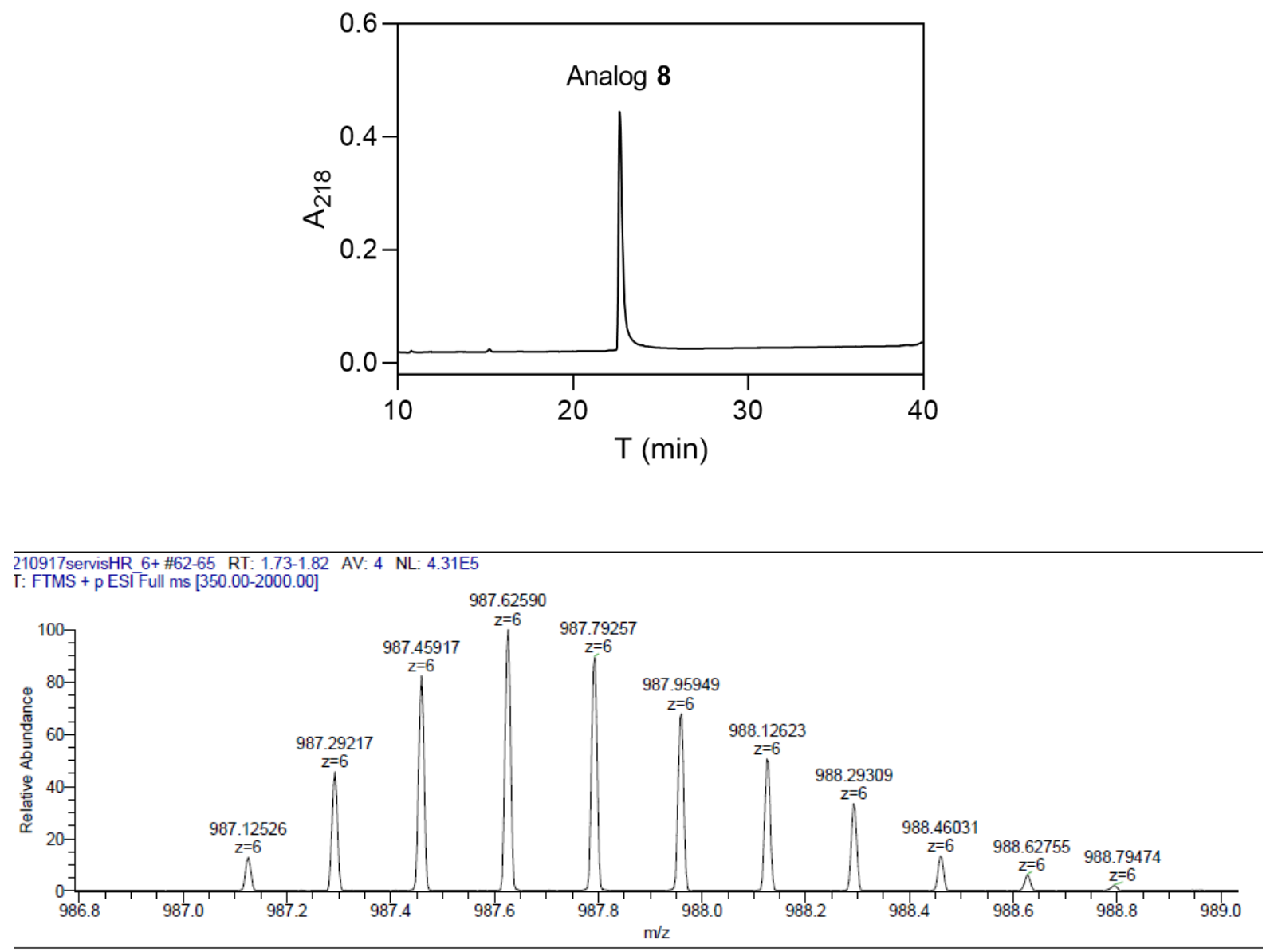

210917servisHR 6+_XT_00001_M_\#2 RT: 2.00 AV: 1 NL: $8.21 E 5$

I: FTMS + p ESĪ Full ms [350.00-20000.00]

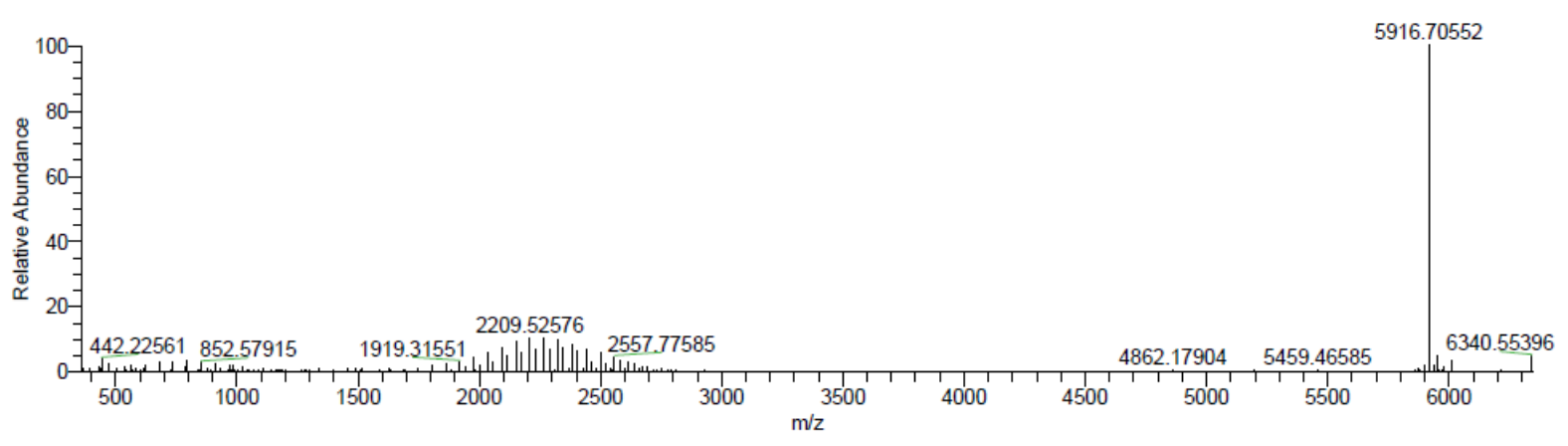

Figure S9. Upper panel: RP-HPLC analysis of analog 8 ([Gly ${ }^{\mathrm{B} 31}$, Gly ${ }^{\mathrm{B} 32}$, amide $\left.{ }^{\mathrm{B} 32}\right]$-insulin). Lower panel: HR-MS of analog 8, Mw (monoisotopic for $\mathrm{C}_{261} \mathrm{H}_{390} \mathrm{~N}_{68} \mathrm{O}_{78} \mathrm{~S}_{6}$ ) calculated 5916.6966, Mw found 5916.7055. 

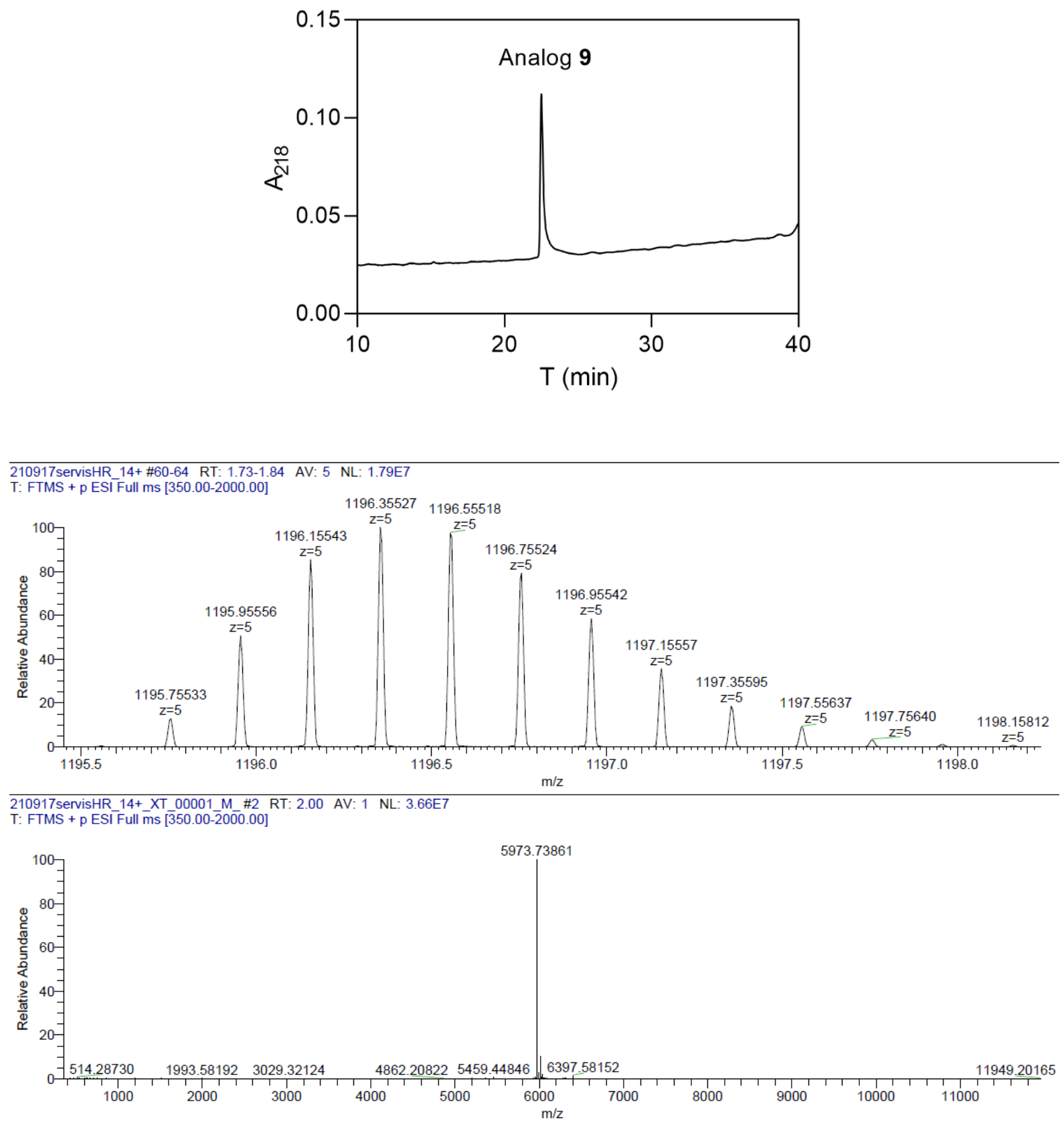

Figure S10. Upper panel: RP-HPLC analysis of analog 9 ([Gly ${ }^{\mathrm{B} 31}, \mathrm{Gly}^{\mathrm{B} 32}, \mathrm{Gly}^{\mathrm{B} 33}$, $\left.\mathrm{amide}^{\mathrm{B} 33}\right]$ insulin). Lower panel: HR-MS of analog 9, $\mathrm{Mw}$ (monoisotopic for $\mathrm{C}_{263} \mathrm{H}_{393} \mathrm{~N}_{69} \mathrm{O}_{79} \mathrm{~S}_{6}$ ) calculated 5973.7180, Mw found 5973.7386. 

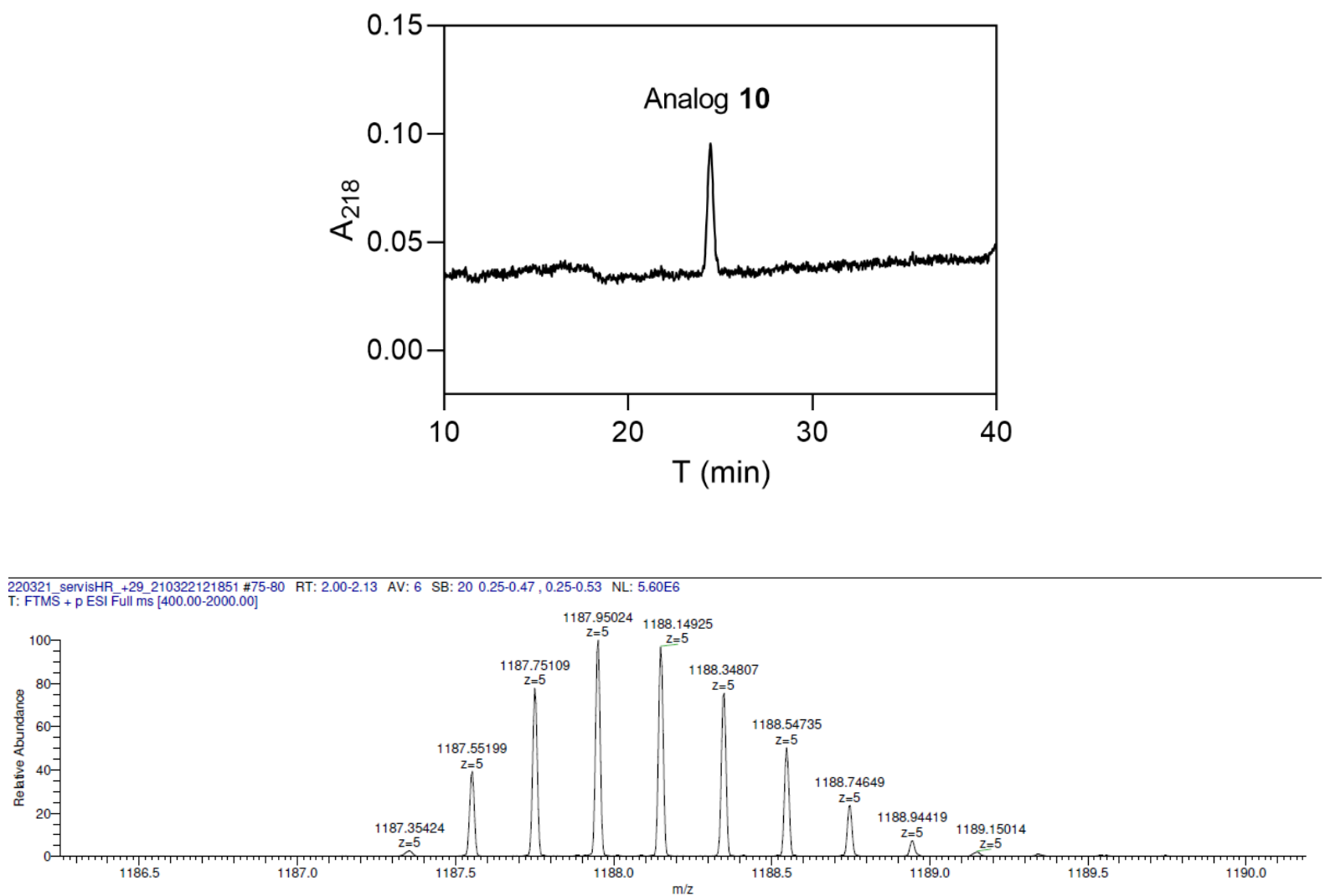

220321_servisHR_+29_210322121851_XT_00001_M_\#2 RT: 2.00 AV: 1 SB: $21.00,1.00$ NL: 8.57E6

T: FTMS + p ESI Full ms $[400.00-2000.00]$

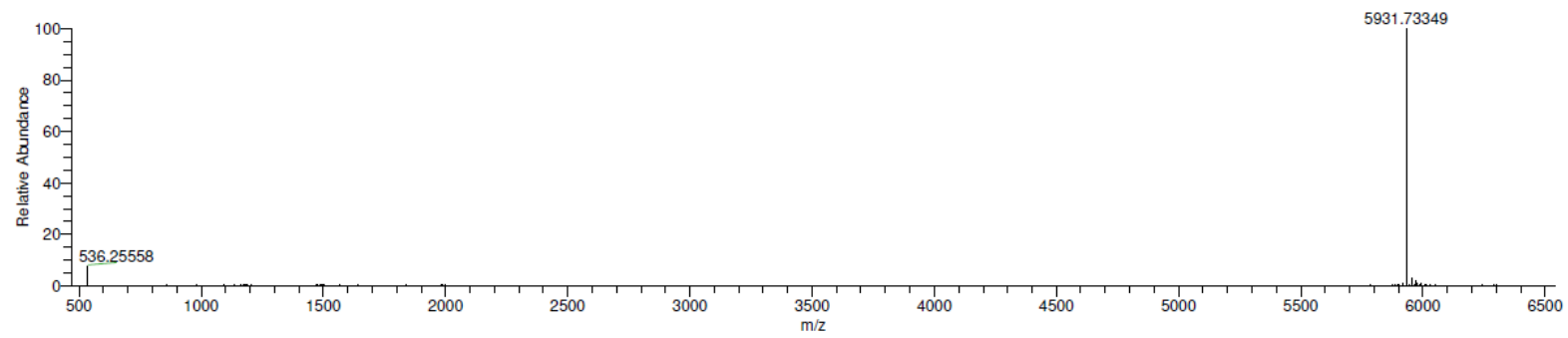

Figure S11. Upper panel: RP-HPLC analysis of analog 10 ([Glu ${ }^{\mathrm{B} 31}$, amide $\left.{ }^{\mathrm{B} 31}\right]$-insulin). Lower panel: HR-MS of analog 10, Mw (monoisotopic for $\mathrm{C}_{262} \mathrm{H}_{391} \mathrm{~N}_{67} \mathrm{O}_{79} \mathrm{~S}_{6}$ ) calculated 5931.6962, Mw found 5931.7335 . 

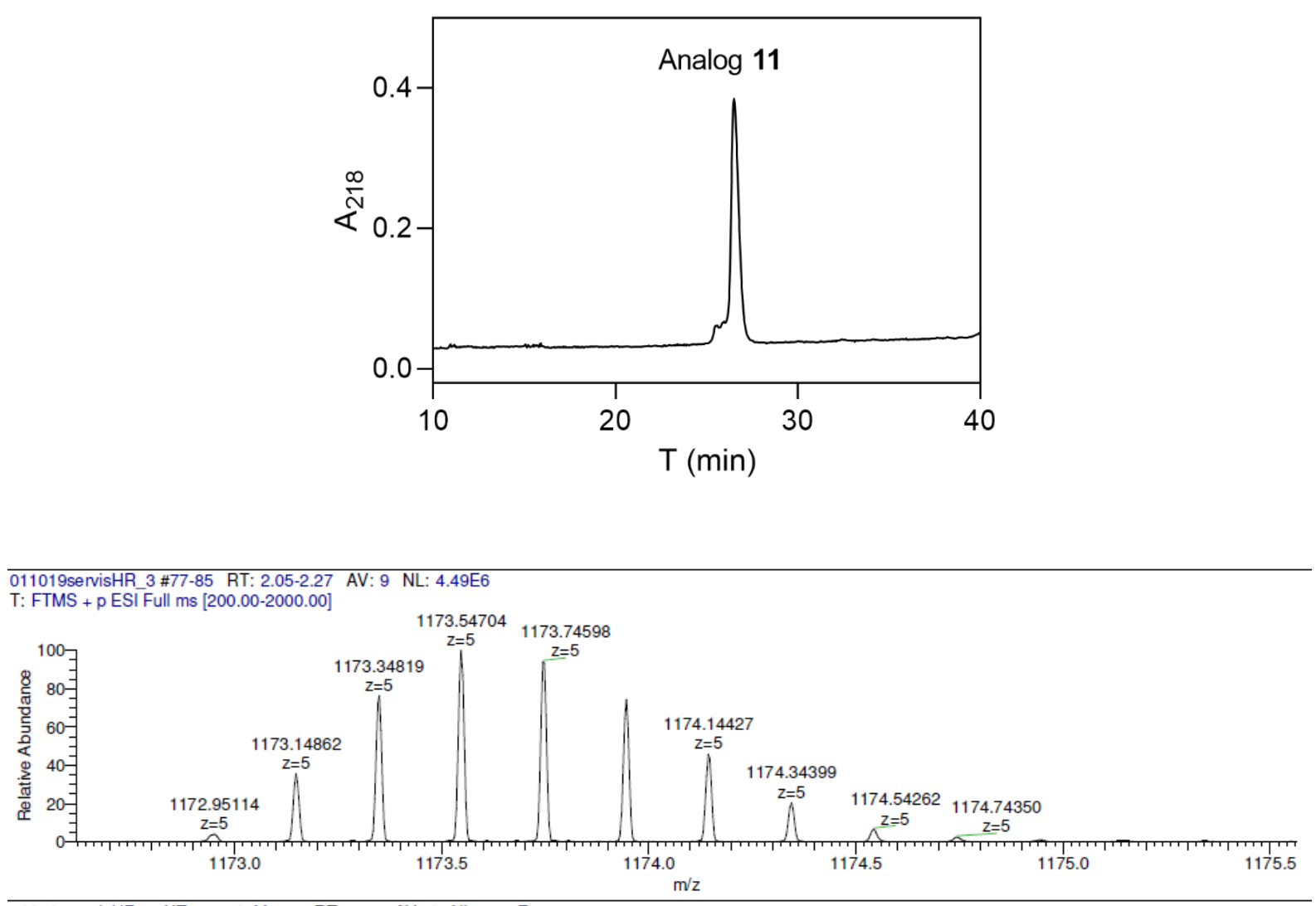

011019servisHR_3_XT_00001_M_\#2 RT: 2.00 AV: 1 NL: $6.40 E 6$

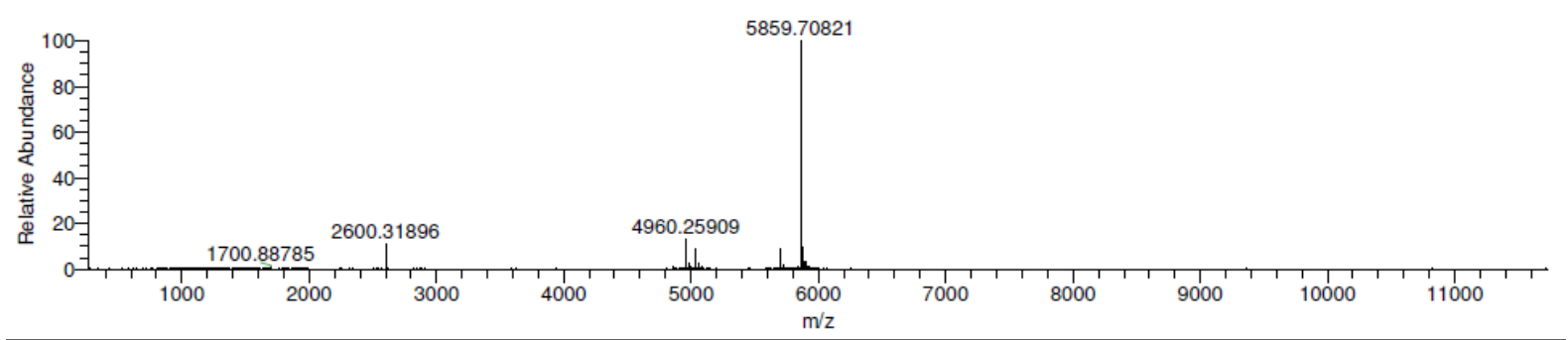

011019servisHR_3_XT_00001_M_\#2 RT: 2.00 AV: 1 NL: 6.40E6

T: FTMS + p ESI Full ms [200.00-2000.00]

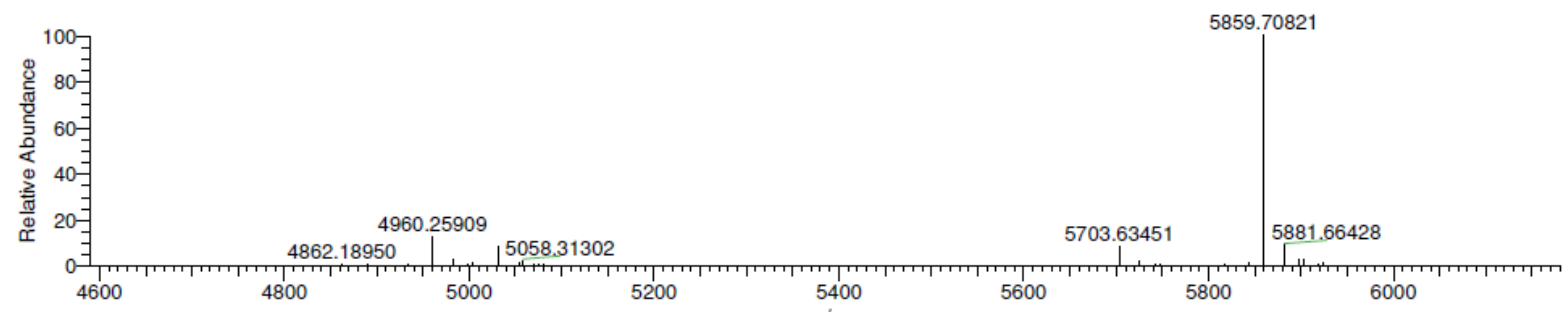

Figure S12. Upper panel: RP-HPLC analysis of analog 11 ([Lys ${ }^{\mathrm{B} 28}$, $\mathrm{Pro}^{\mathrm{B} 29}, \mathrm{Gly}^{\mathrm{B} 31}$, amide $\left.{ }^{\mathrm{B} 31}\right]$ insulin). Lower panel: HR-MS of analog 11, Mw (monoisotopic for $\mathrm{C}_{259} \mathrm{H}_{387} \mathrm{~N}_{67} \mathrm{O}_{77} \mathrm{~S}_{6}$ ) calculated 5859.6751, Mw found 5859.7082. 

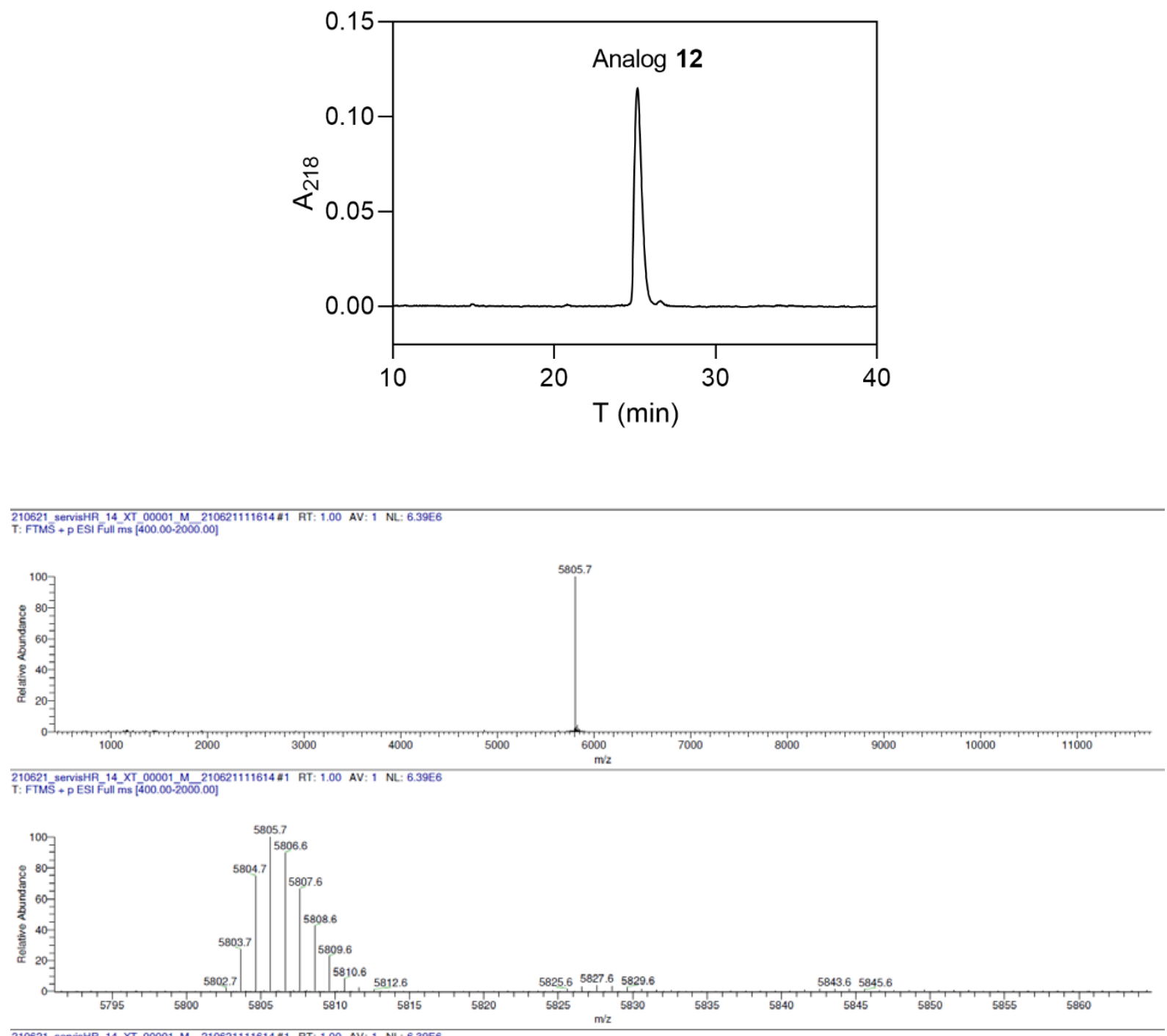

210621_servisHR_14_XT_00001_M_210621111614 \#1 RT: 1.00 AV: 1 NL: 6.39E6
T: FTMS + D ESI Full ms [400.00-2000.00]

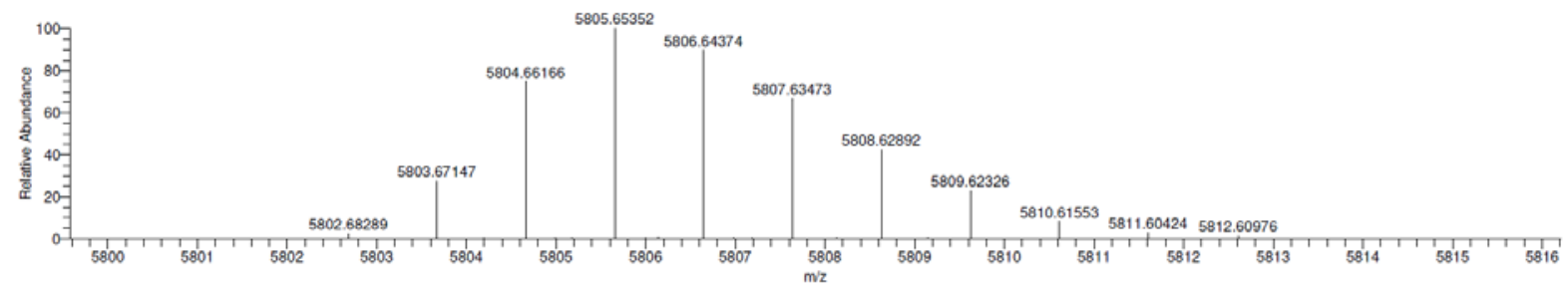

Figure S13. Upper panel: RP-HPLC analysis of analog 12 ([Ala ${ }^{\mathrm{B} 29}$, $\mathrm{Gly}^{\mathrm{B} 31}$, amide $\left.{ }^{\mathrm{B} 31}\right]$-insulin). Lower panel: HR-MS of analog 12, Mw (monoisotopic for $\mathrm{C}_{256} \mathrm{H}_{380} \mathrm{~N}_{66} \mathrm{O}_{77} \mathrm{~S}_{6}$ ) calculated 5802.6172, Mw found 5802.6829. 

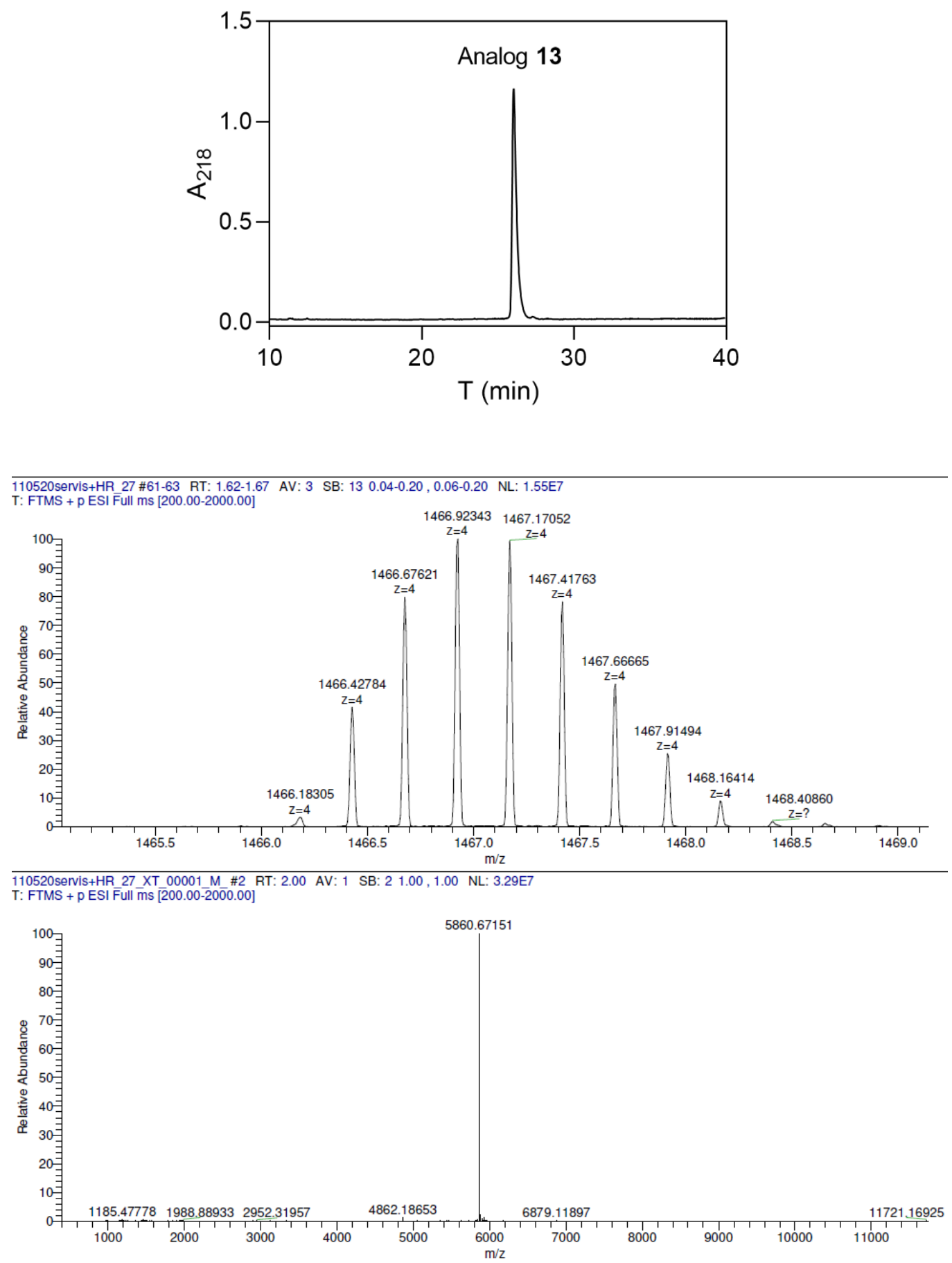

Figure S14. Upper panel: RP-HPLC analysis of analog 13 ([Ala ${ }^{\mathrm{B} 29}, \mathrm{Asp}^{\mathrm{B} 31}$, amide $\left.^{\mathrm{B} 31}\right]$-insulin). Lower panel: HR-MS of analog 13, Mw (monoisotopic for $\mathrm{C}_{258} \mathrm{H}_{382} \mathrm{~N}_{66} \mathrm{O}_{79} \mathrm{~S}_{6}$ ) calculated 5860.6227, Mw found 5860.6715. 

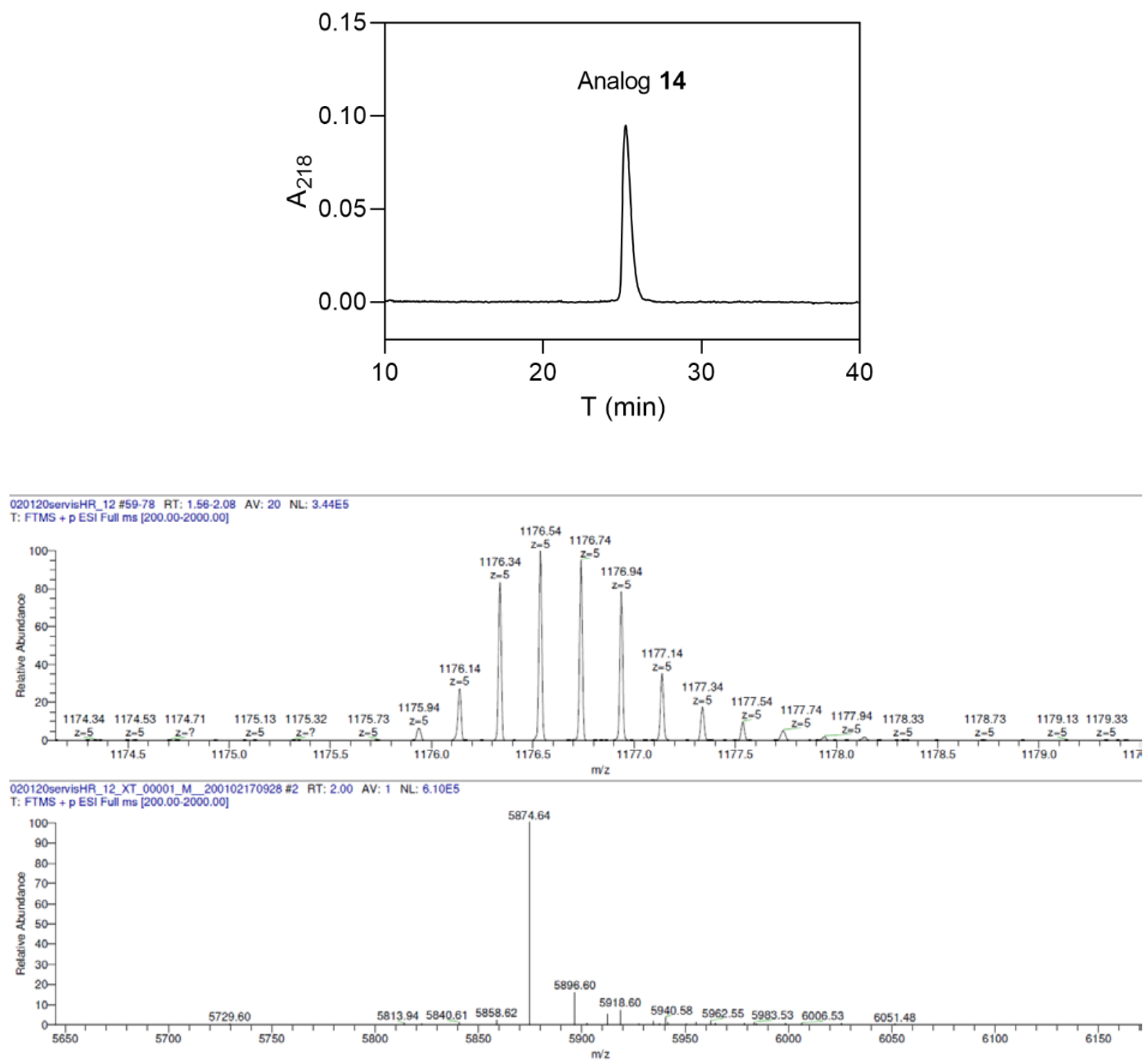

Figure S15. Upper panel: RP-HPLC analysis of analog 14 ([Ala ${ }^{\mathrm{B} 29}, \mathrm{Glu}^{\mathrm{B} 31}$, amide $\left.{ }^{\mathrm{B} 31}\right]$-insulin). Lower panel: HR-MS of analog 14, Mw (monoisotopic for $\mathrm{C}_{259} \mathrm{H}_{384} \mathrm{~N}_{66} \mathrm{O}_{79} \mathrm{~S}_{6}$ ) calculated 5874.6384, Mw found 5874.64. 

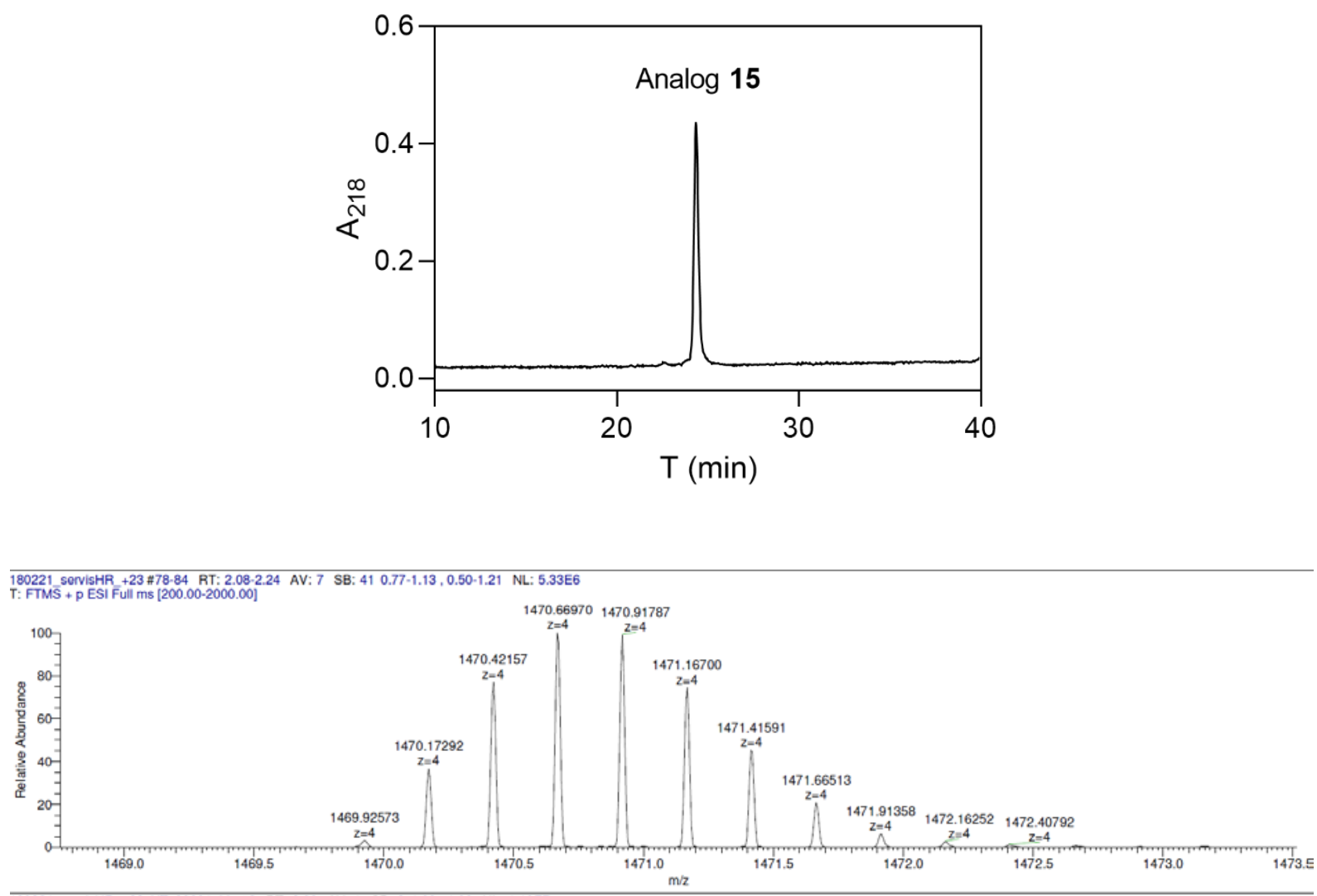

180221_servisHR_+23_XT_00001_M_\#2 RT: 2.00 AV: 1 SB: $21.00,1.00$ NL: $1.10 E 7$

$\mathrm{T}: \mathrm{FTM} \overline{\mathrm{S}}+\mathrm{p}$ ESI Full ms $(200.00-200 \overline{0} .00)$

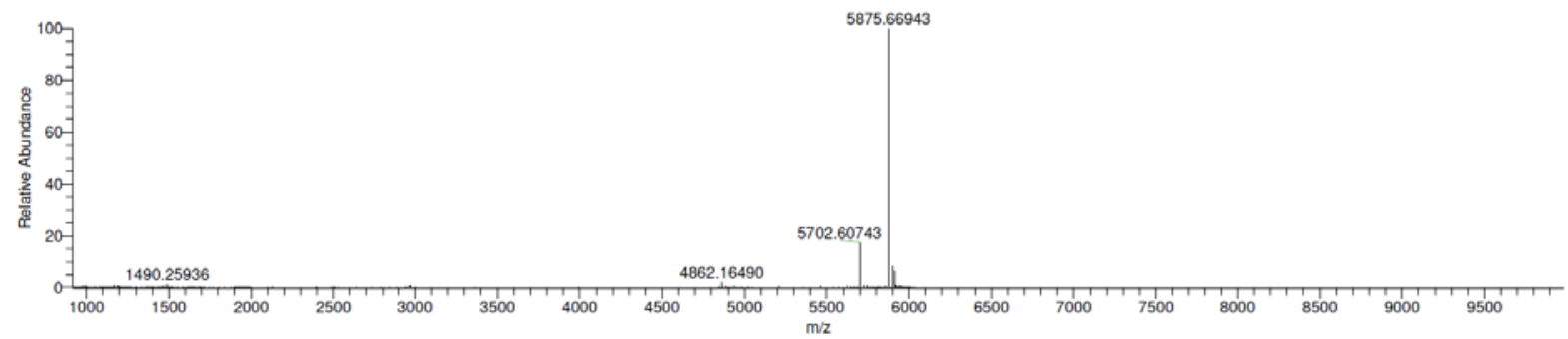

Figure S16. Upper panel: RP-HPLC analysis of analog 15 ([Ala $\left.{ }^{\mathrm{B} 29}, \mathrm{Glu}^{\mathrm{B} 31}\right]$-insulin). Lower panel: HR-MS of analog 15, Mw (monoisotopic for $\mathrm{C}_{259} \mathrm{H}_{383} \mathrm{~N}_{65} \mathrm{O}_{80} \mathrm{~S}_{6}$ ) calculated 5875.6224, Mw found 5875.6694. 


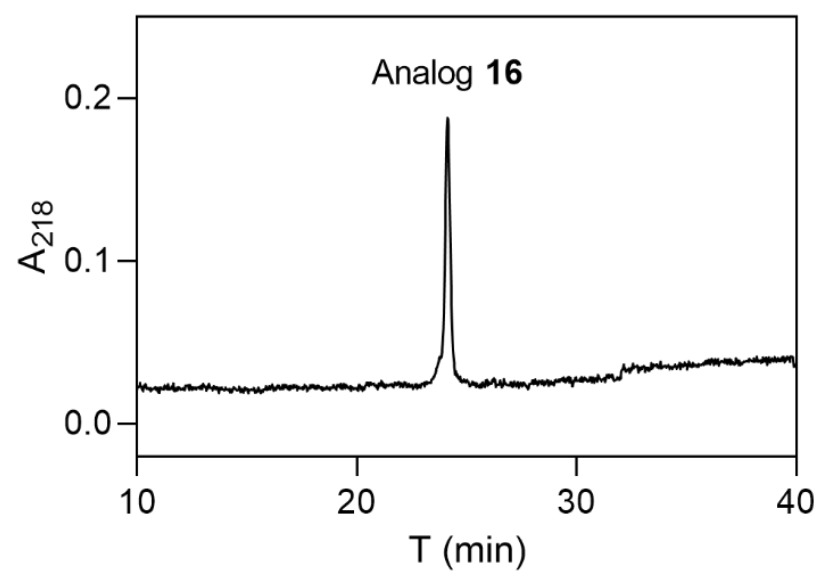

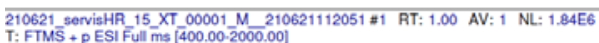

$T: F T M S+P$ ESI Fuif

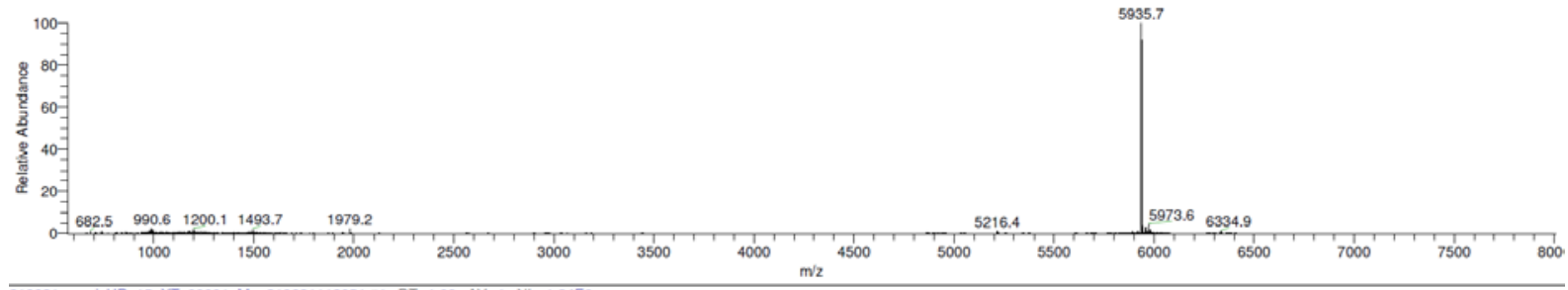

210621 servisHR_15_XT 00001 M 210621112051 \#1 RT: 1.00 AV: 1 NL: $1.84 E 6$
T:FTMS + P ESI Full ms [400.00-2000.00]

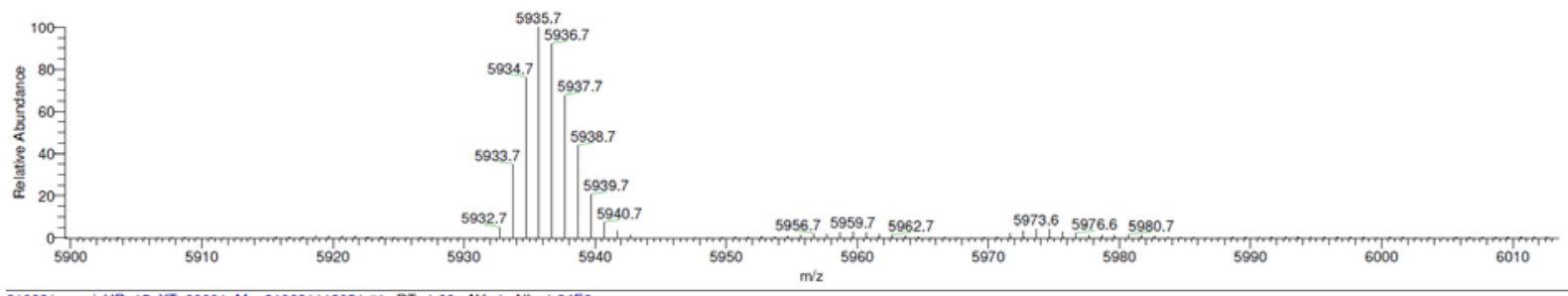

210621 servisHR 15 XT 00001 M 210621112051 \#1 RT: 1.00 AV: 1 NL: $1.84 E 6$
T: FTMS + P ESI Full ms $400.00-2000.00]$

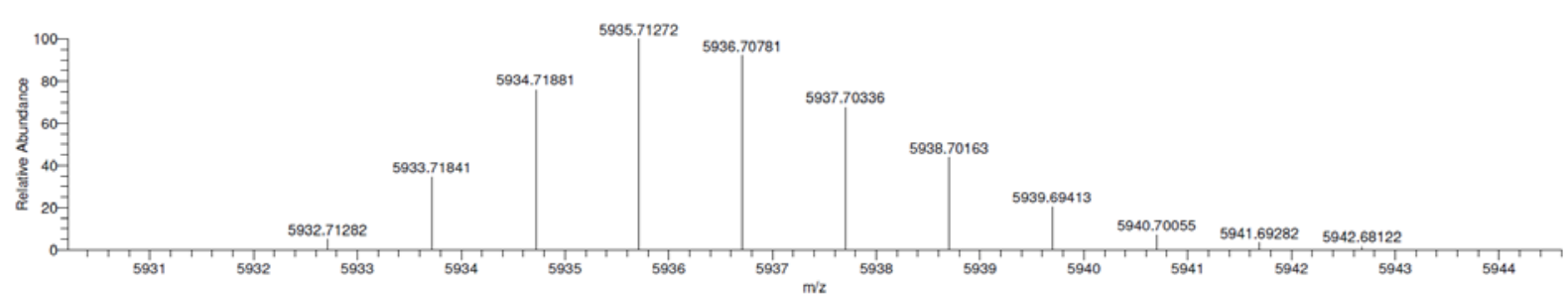

Figure S17. Upper panel: RP-HPLC analysis of analog 16 ([Lys ${ }^{\mathrm{B} 28}$, Pro $\left.{ }^{\mathrm{B} 29}, \mathrm{Glu}^{\mathrm{B} 31}\right]$-insulin). Lower panel: HR-MS of analog 16, Mw (monoisotopic for $\mathrm{C}_{262} \mathrm{H}_{390} \mathrm{~N}_{66} \mathrm{O}_{80} \mathrm{~S}_{6}$ ) calculated 5932.6802, Mw found 5932.7128. 

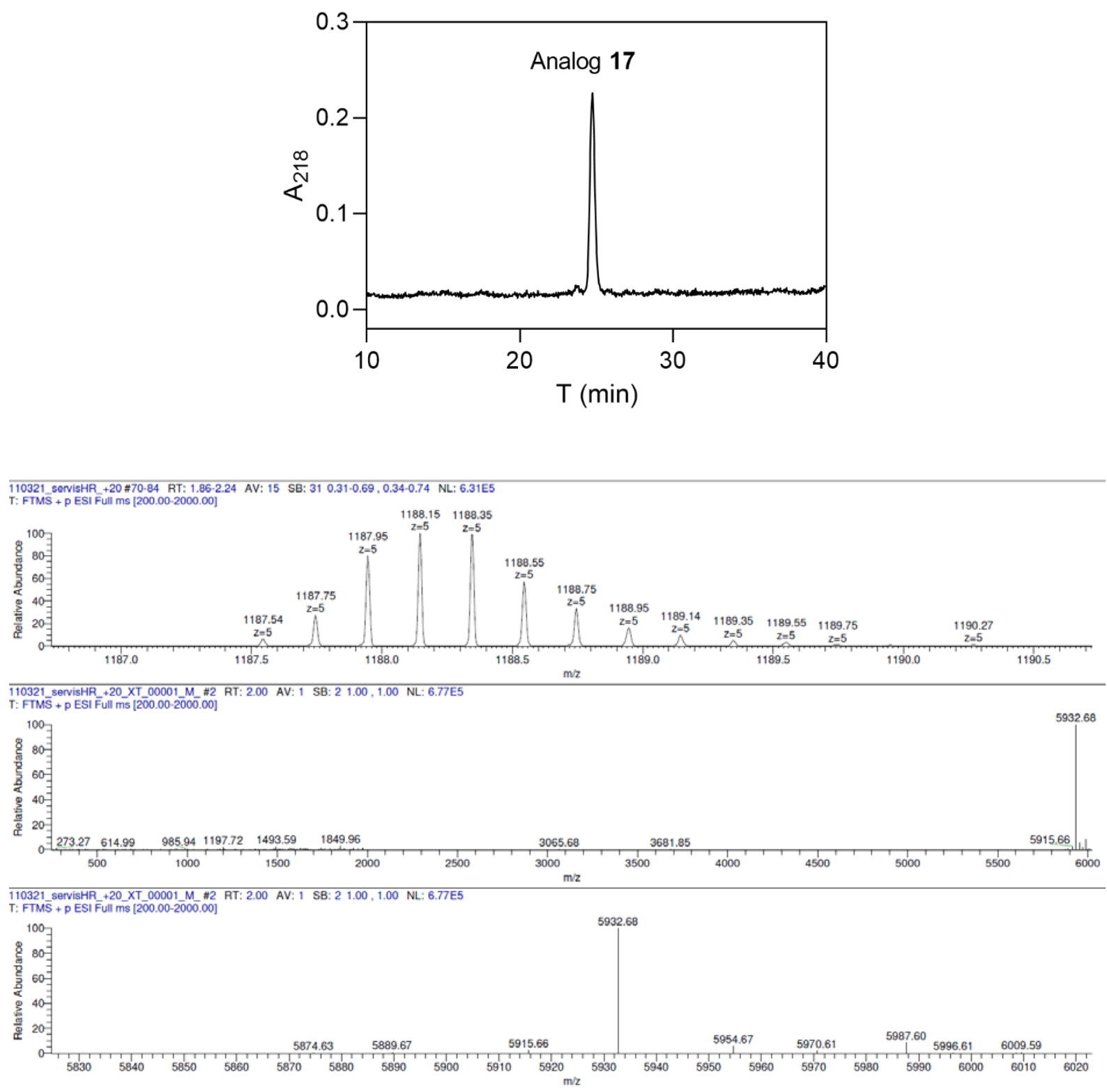

Figure S18. Upper panel: RP-HPLC analysis of analog 17 ([Glu $\left.{ }^{\mathrm{B} 31}\right]$-insulin). Lower panel: HRMS of analog 17, Mw (monoisotopic for $\mathrm{C}_{262} \mathrm{H}_{390} \mathrm{~N}_{66} \mathrm{O}_{80} \mathrm{~S}_{6}$ ) calculated 5932.6802, Mw found 5932.68. 

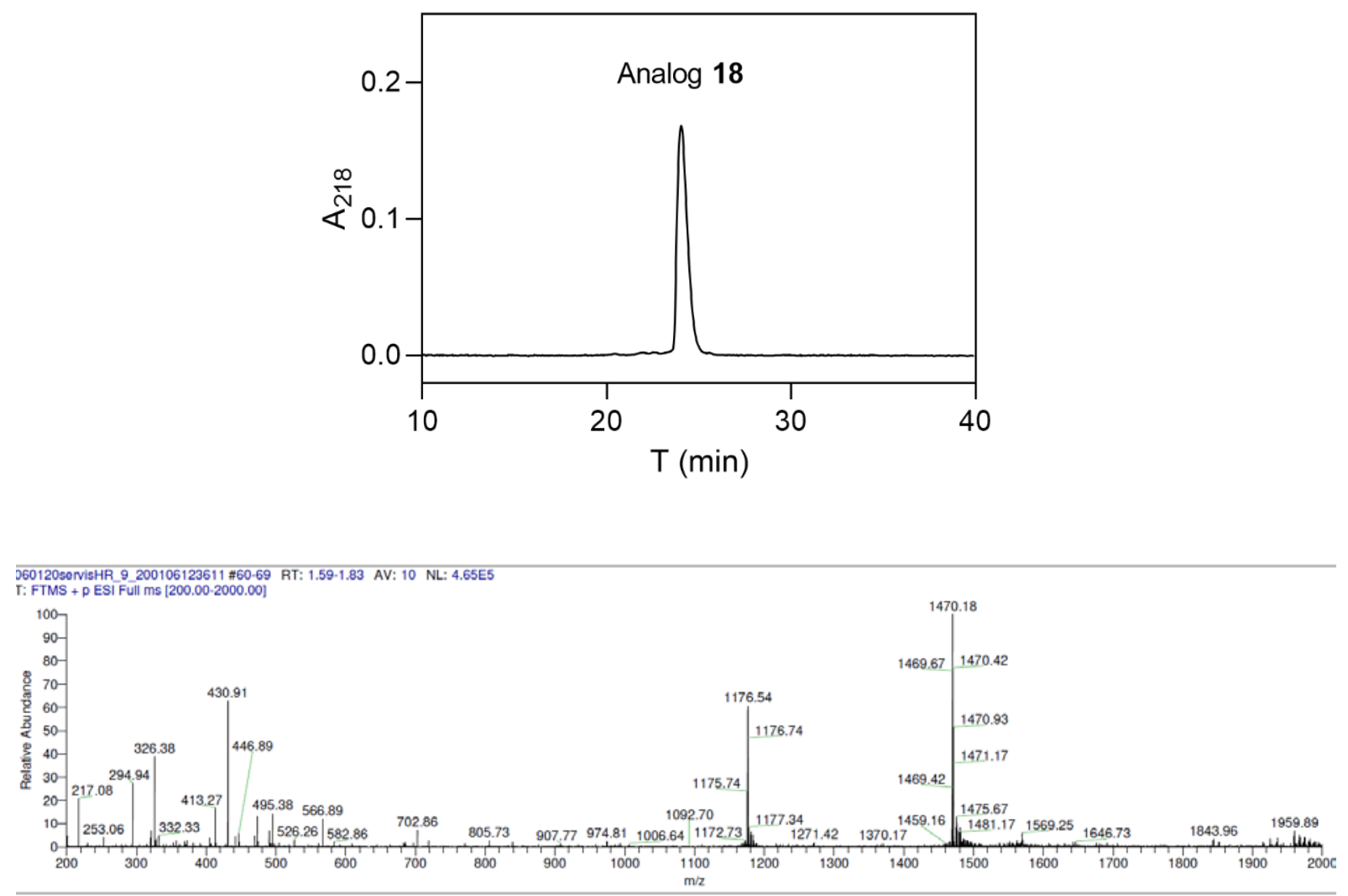

J60120servisHR_9_200106123611 \#58-74 RT: 1.53-1.97 AV: $17 \quad$ NL: 2.06E5
T: FTMS + p ESI Full ms [200.00-2000.00]
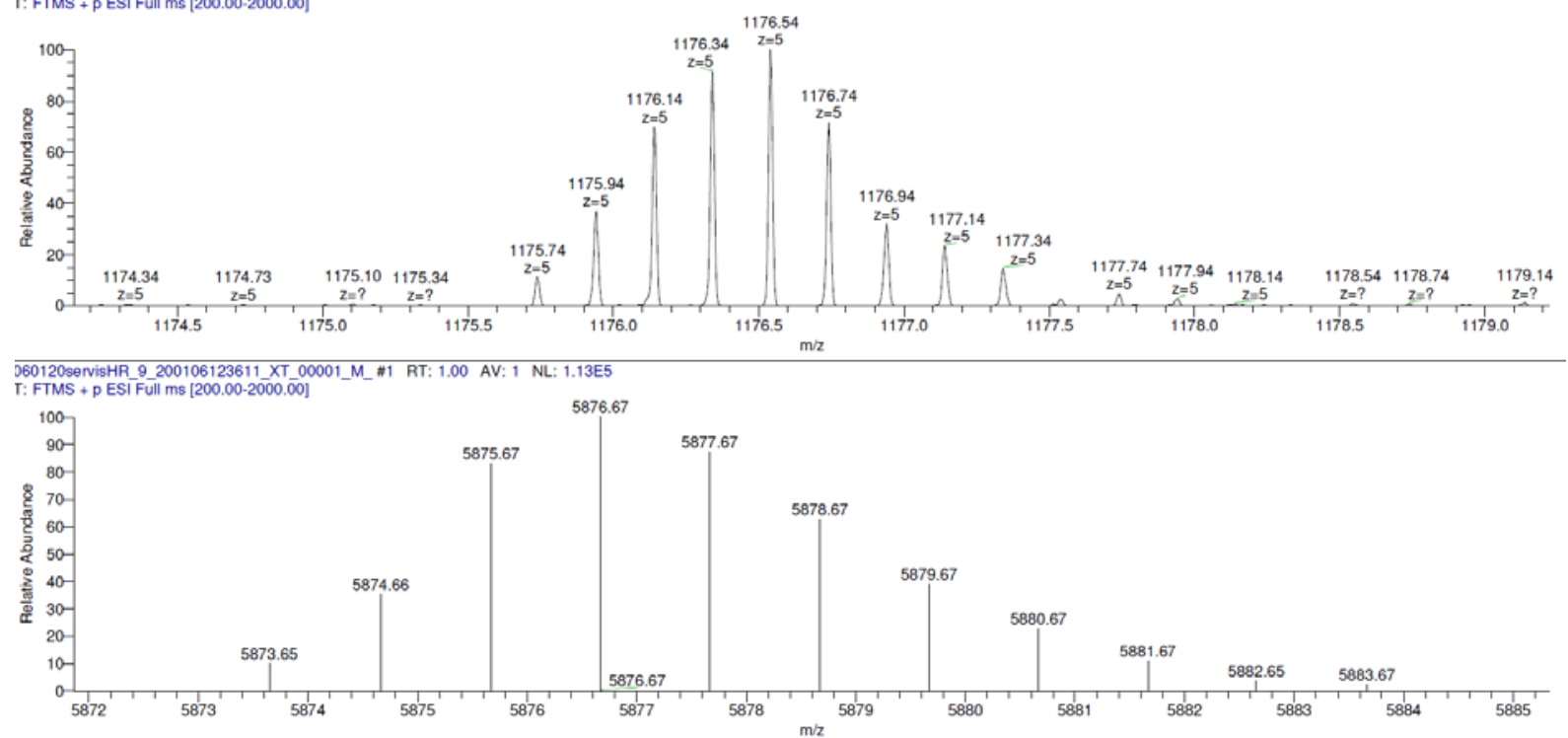

Figure S19. Upper panel: RP-HPLC analysis of analog 18 ([Ala ${ }^{\mathrm{B} 29}, \mathrm{Gln}^{\mathrm{B} 31}$, amide $\left.{ }^{\mathrm{B} 31}\right]$-insulin). Lower panel: HR-MS of analog 18, Mw (monoisotopic for $\mathrm{C}_{259} \mathrm{H}_{385} \mathrm{~N}_{67} \mathrm{O}_{78} \mathrm{~S}_{6}$ ) calculated 5873.6544, Mw found 5873.65. 

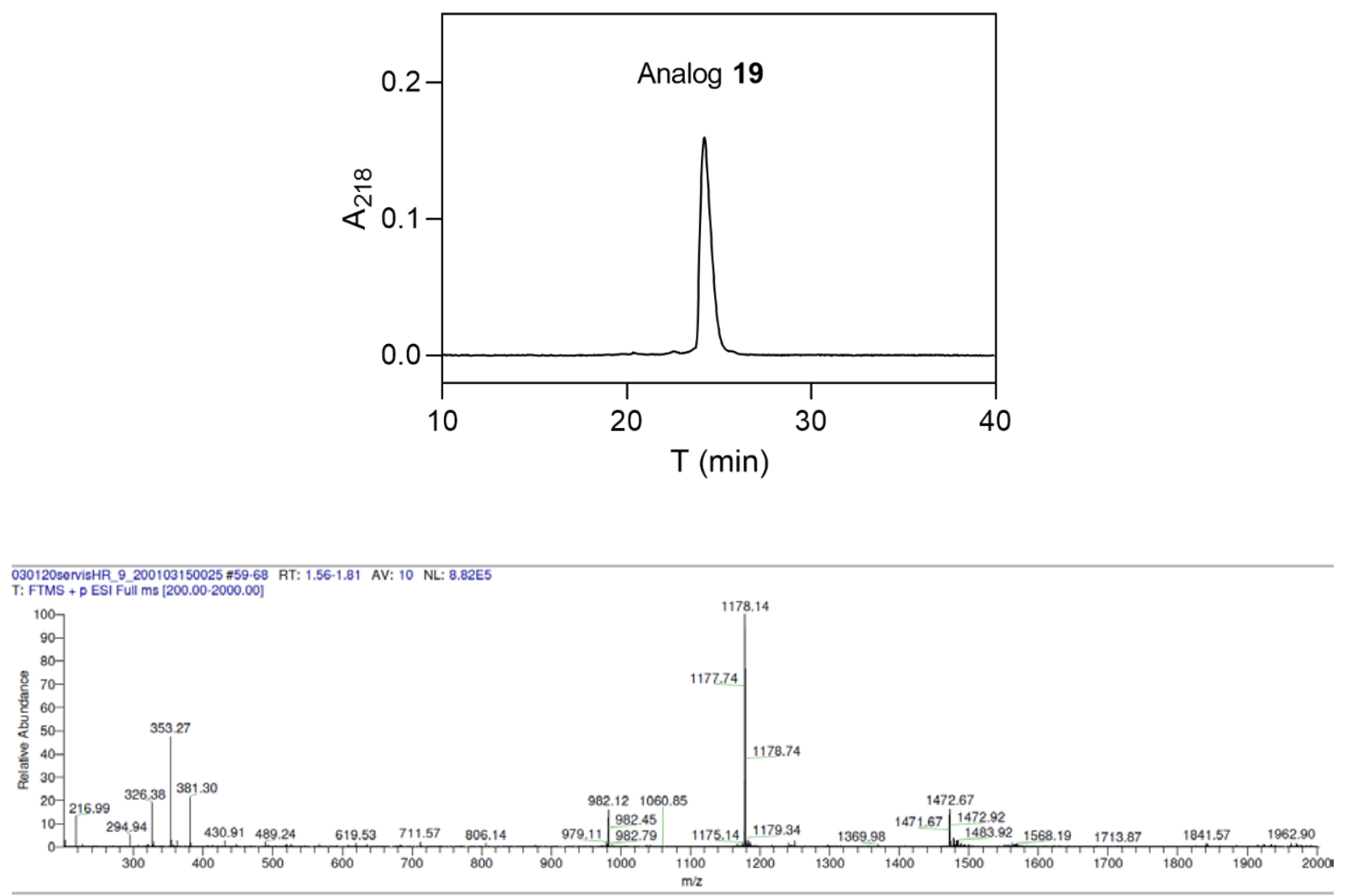

030120servisHR_9_200103150025 \#59-68 RT: 1.56-1.81 AV: 10 NL: 8.82ES

T: FTMS + P ESI Füll ms [200,0025

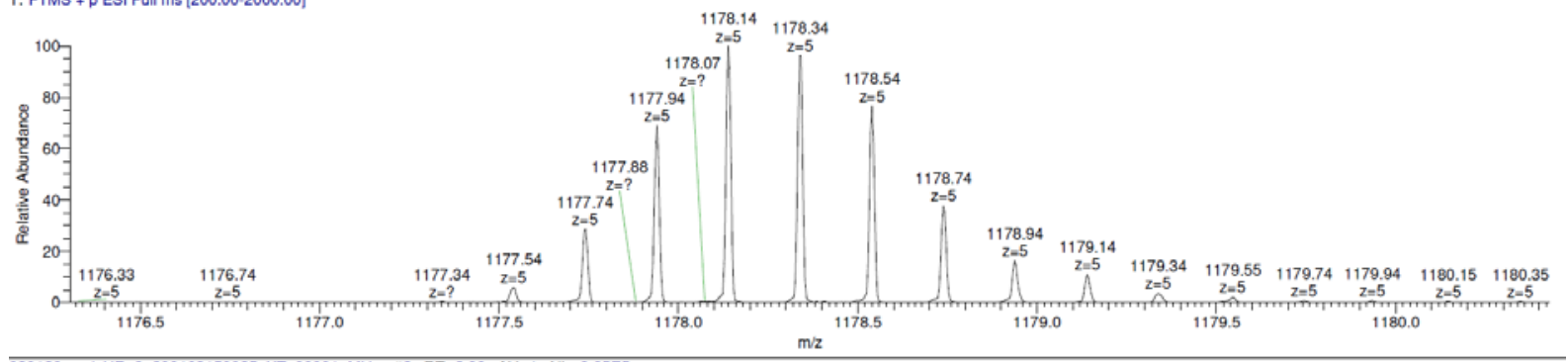

030120servisHR_9_200103150025_XT_00001_MHP_"\#2 RT: 2.00 AV: 1 NL: 9.95E5

T: FTMS + p ESI Füll ms [200.00-200

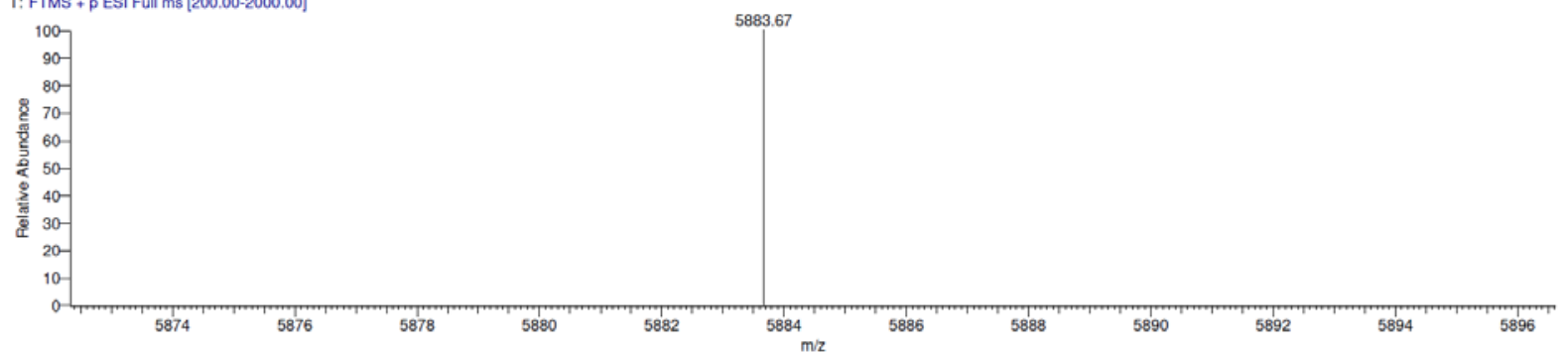

Figure S20. Upper panel: RP-HPLC analysis of analog 19 ([Ala ${ }^{\mathrm{B} 29}, \mathrm{His}^{\mathrm{B} 31}$, amide $\left.{ }^{\mathrm{B} 31}\right]$-insulin). Lower panel: HR-MS of analog 19, Mw (monoisotopic for $\mathrm{C}_{260} \mathrm{H}_{384} \mathrm{~N}_{68} \mathrm{O}_{77} \mathrm{~S}_{6}$ ) calculated 5882.6547, Mw found 5882.66 (5883.67 for $\mathrm{MH}^{+}$). 

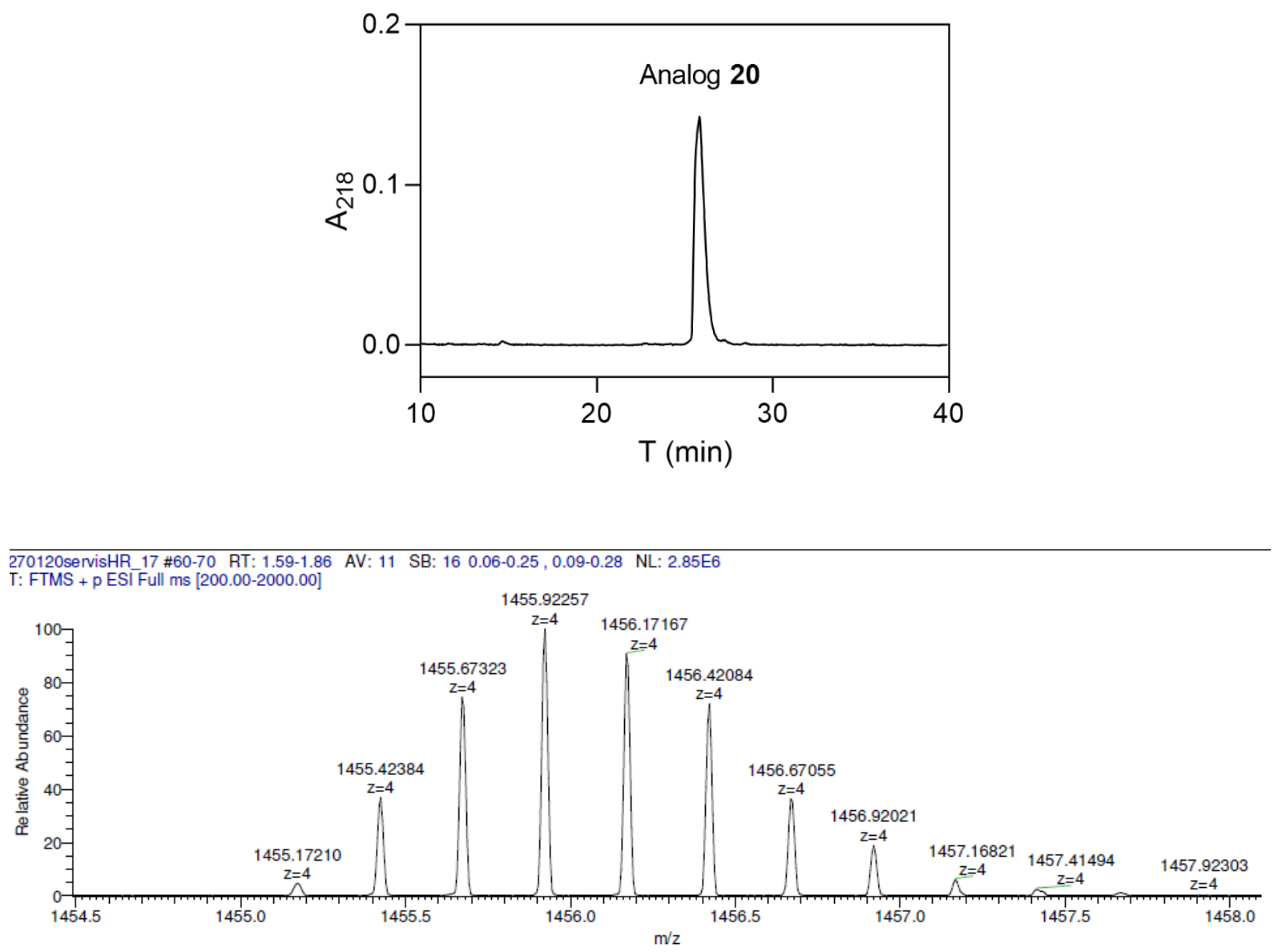

270120servisHR_17_XT_00001_M_\#2 RT: 2.00 AV: 1 SB: 2 1.00,1.00 NL: 5.15E6

T: FTMS + p ESII Full ms [200.00-2000.00]

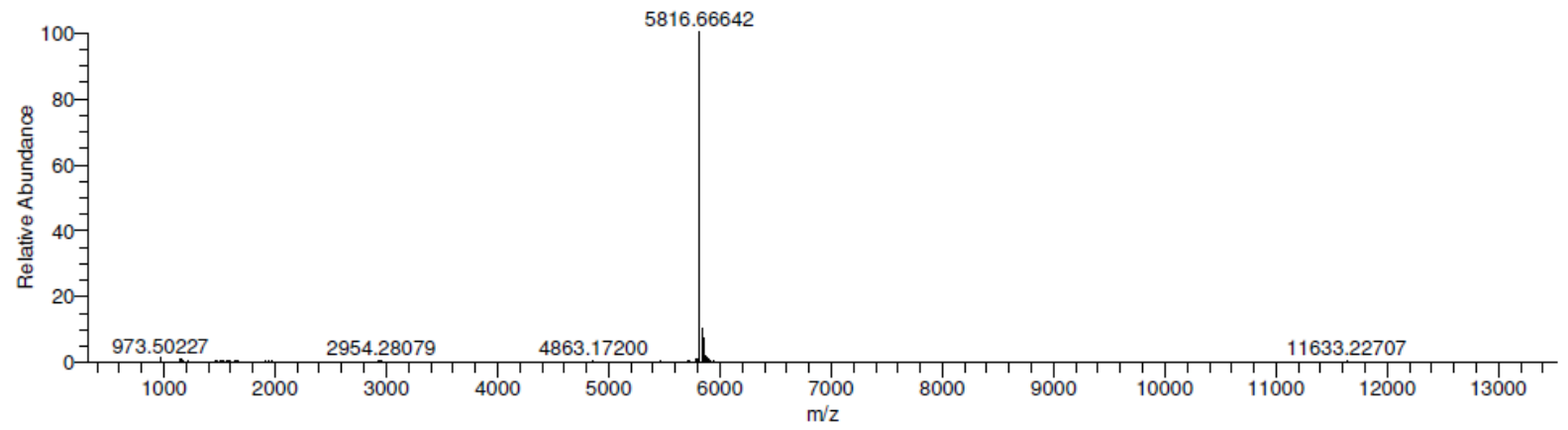

Figure S21. Upper panel: RP-HPLC analysis of analog 20 ([Ala ${ }^{\mathrm{B} 29}, \mathrm{Ala}^{\mathrm{B} 31}$, amide $\left.{ }^{\mathrm{B} 31}\right]$-insulin). Lower panel: HR-MS of analog 20, Mw (monoisotopic for $\mathrm{C}_{257} \mathrm{H}_{382} \mathrm{~N}_{66} \mathrm{O}_{77} \mathrm{~S}_{6}$ ) calculated 5816.6329, Mw found 5816.6664. 

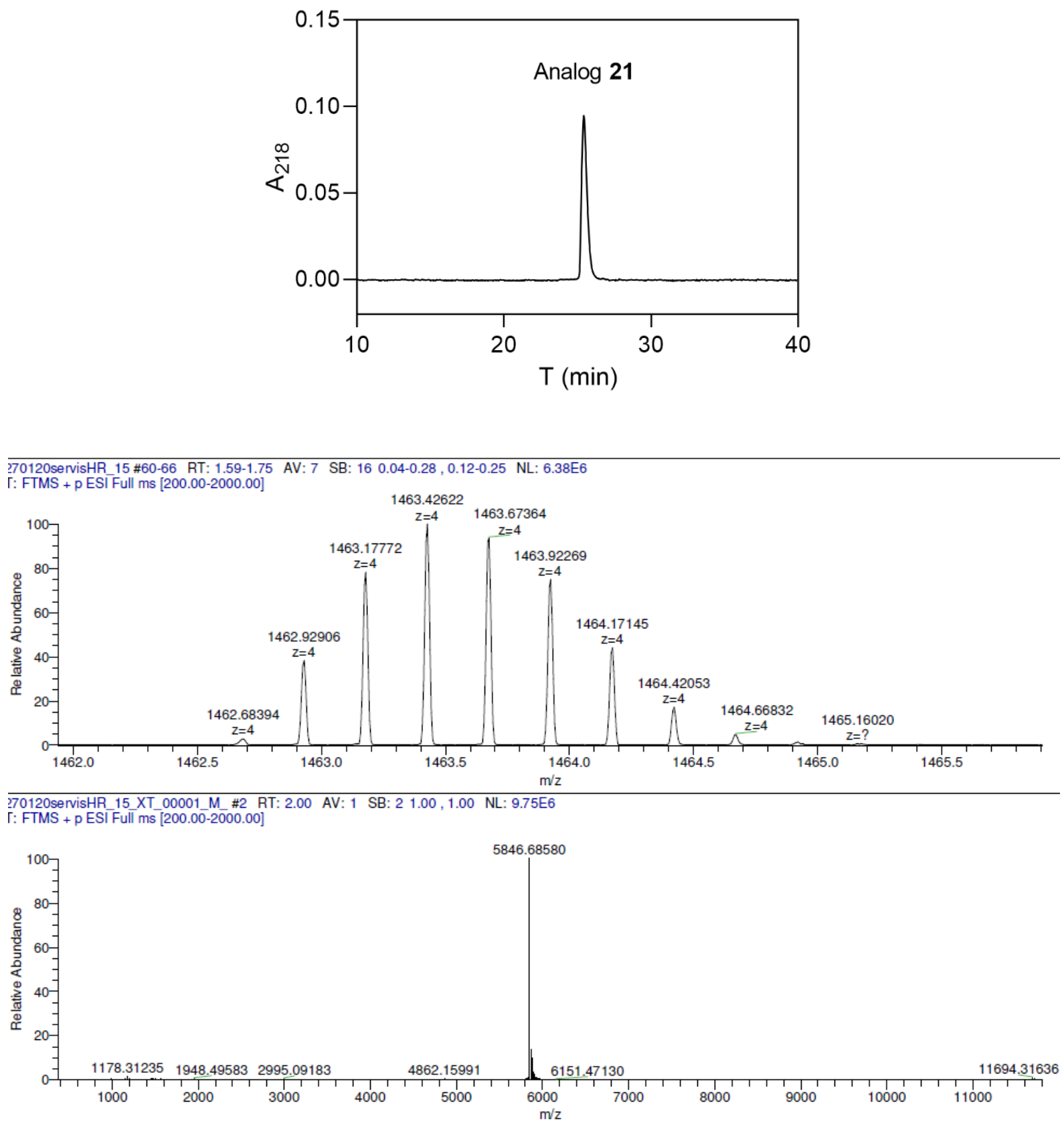

Figure S22. Upper panel: RP-HPLC analysis of analog 21 ([Ala ${ }^{\mathrm{B} 29}$, $\mathrm{Thr}^{\mathrm{B} 31}$, amide $\left.{ }^{\mathrm{B} 31}\right]$-insulin). Lower panel: HR-MS of analog 21, Mw (monoisotopic for $\mathrm{C}_{258} \mathrm{H}_{384} \mathrm{~N}_{66} \mathrm{O}_{78} \mathrm{~S}_{6}$ ) calculated 5846.6435, Mw found 5846.6858. 

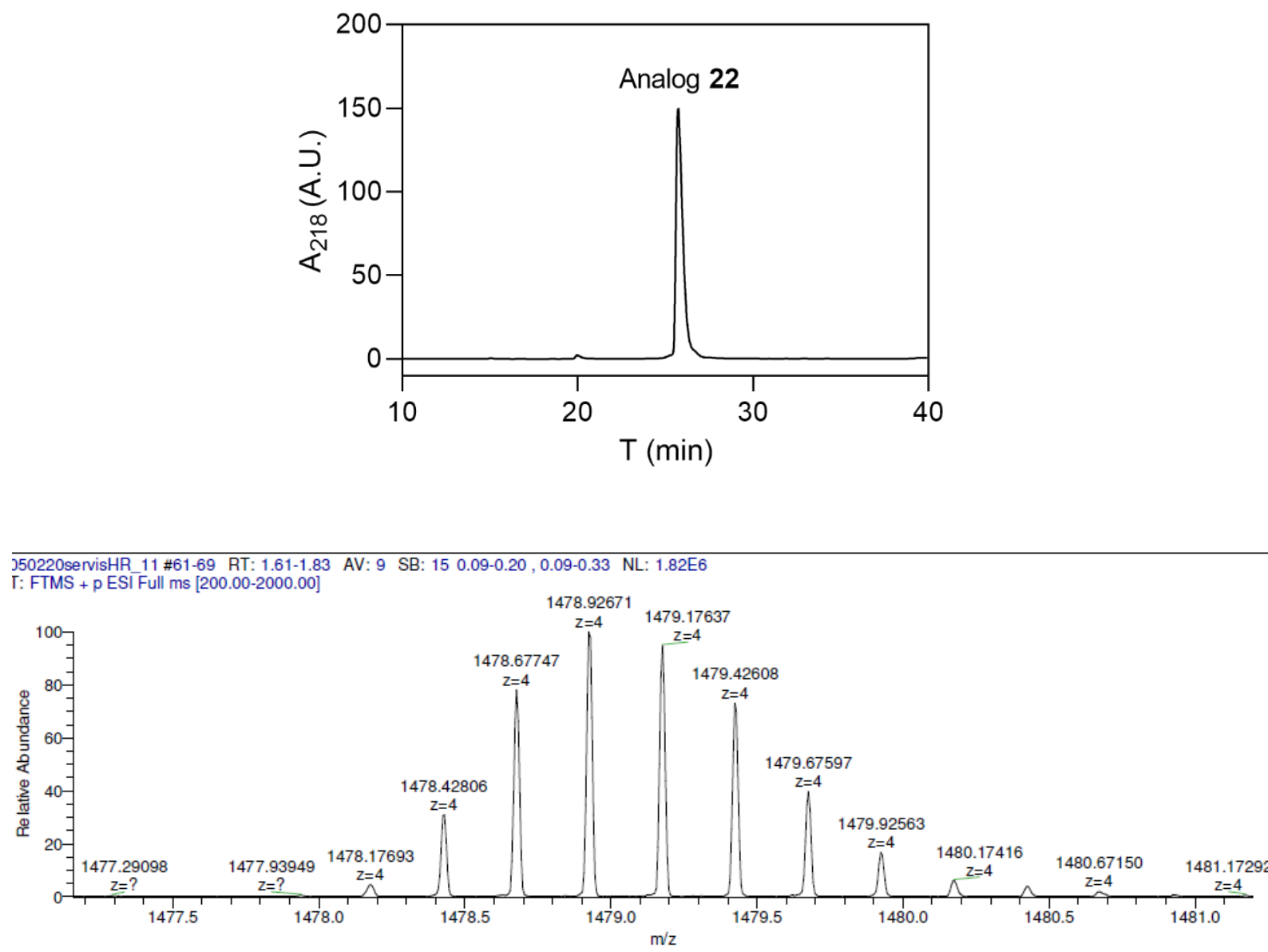

550220servisHR_11_XT_00001_M_\#2 RT: 2.00 AV: 1 SB: 2 1.00,1.00 NL: 3.26E6 T: FTMS + p ESI Full ms [200.00-2000.00]

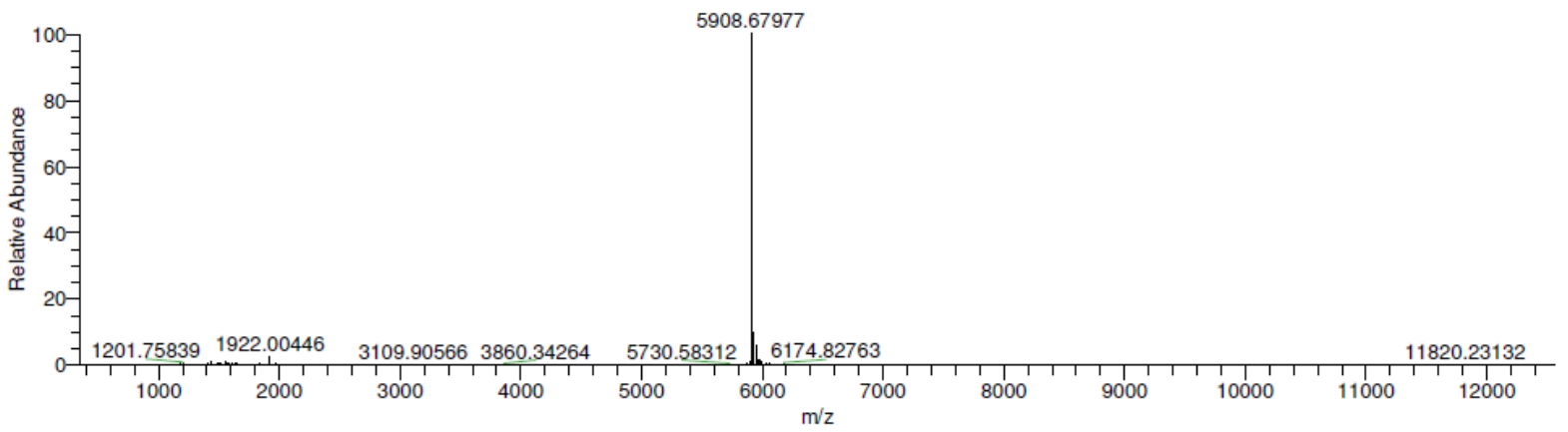

Figure S23. Upper panel: RP-HPLC analysis of analog 22 ([Ala ${ }^{\mathrm{B} 29}, \mathrm{Tyr}^{\mathrm{B} 31}$, amide $\left.{ }^{\mathrm{B} 31}\right]$-insulin). Lower panel: HR-MS of analog 22, $\mathrm{Mw}$ (monoisotopic for $\mathrm{C}_{263} \mathrm{H}_{386} \mathrm{~N}_{66} \mathrm{O}_{78} \mathrm{~S}_{6}$ ) calculated 5908.6591, Mw found 5908.6798. 


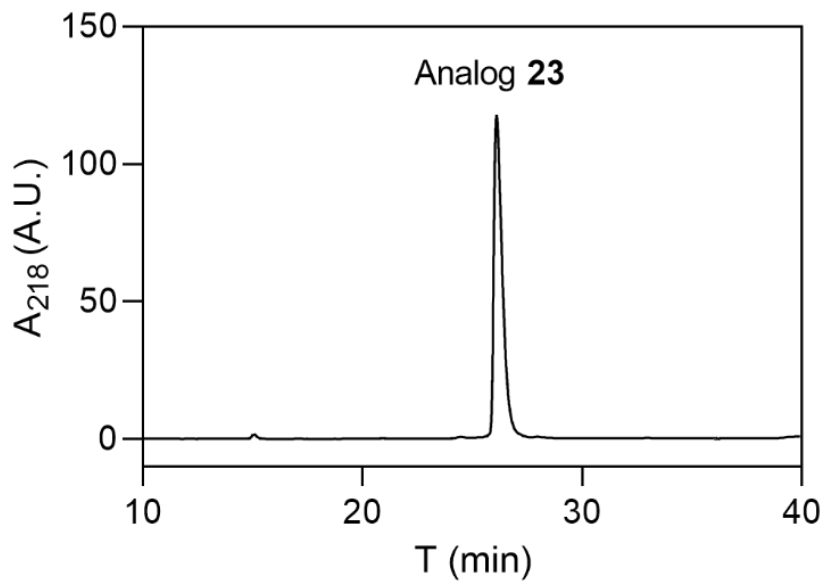

140220servisHR_2 \#60-68 RT: 1.59-1.80 AV: 9 NL: 1.09E7

T: FTMS + p ESI Full ms [200.00-2000.00]

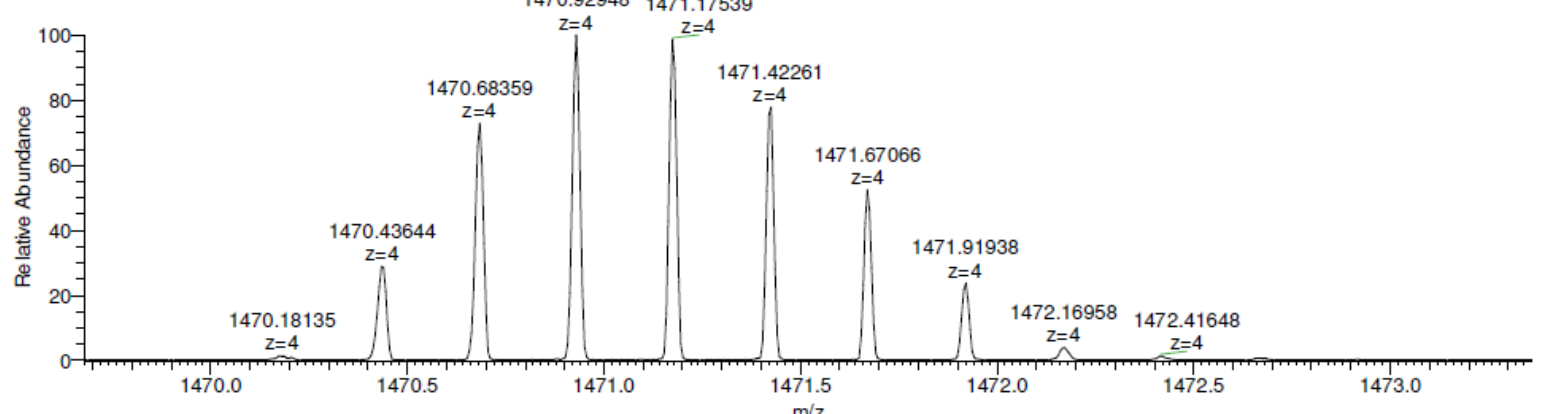

140220servisHR_2_XT_00001_M_\#2 RT: 2.00 AV: 1 NL: 1.72E7

T: FTMS + p ESI Full ms [200.00-2000.00]

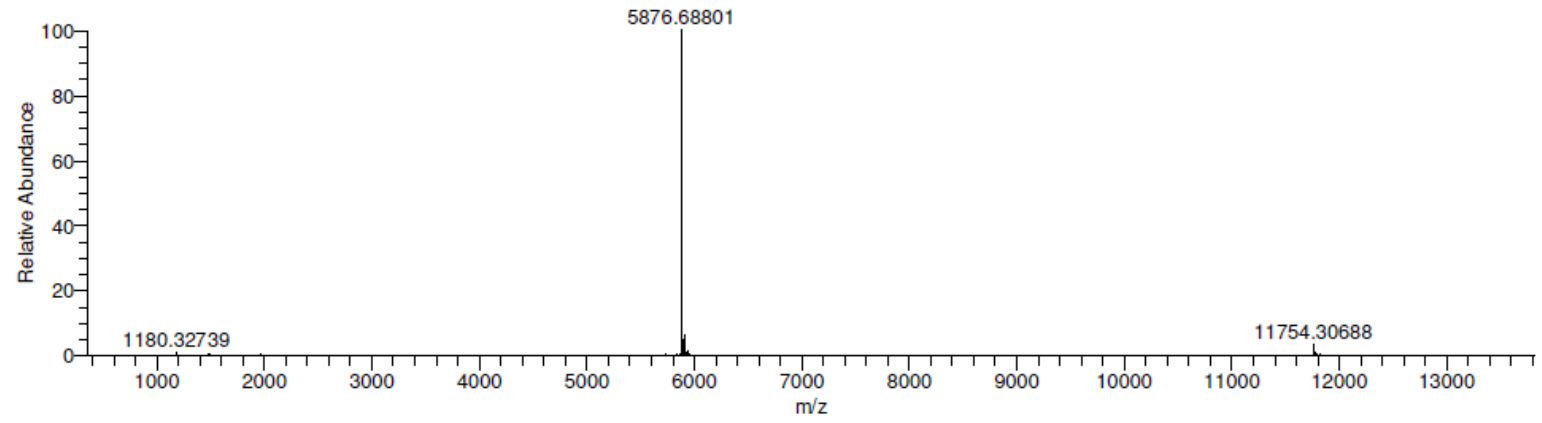

Figure S24. Upper panel: RP-HPLC analysis of analog 23 ([Ala ${ }^{\mathrm{B} 29}, \mathrm{Met}^{\mathrm{B} 31}$, amide $\left.{ }^{\mathrm{B} 31}\right]$-insulin). Lower panel: HR-MS of analog 23, Mw (monoisotopic for $\mathrm{C}_{259} \mathrm{H}_{386} \mathrm{~N}_{66} \mathrm{O}_{77} \mathrm{~S}_{7}$ ) calculated 5876.6363, Mw found 5876.6880. 

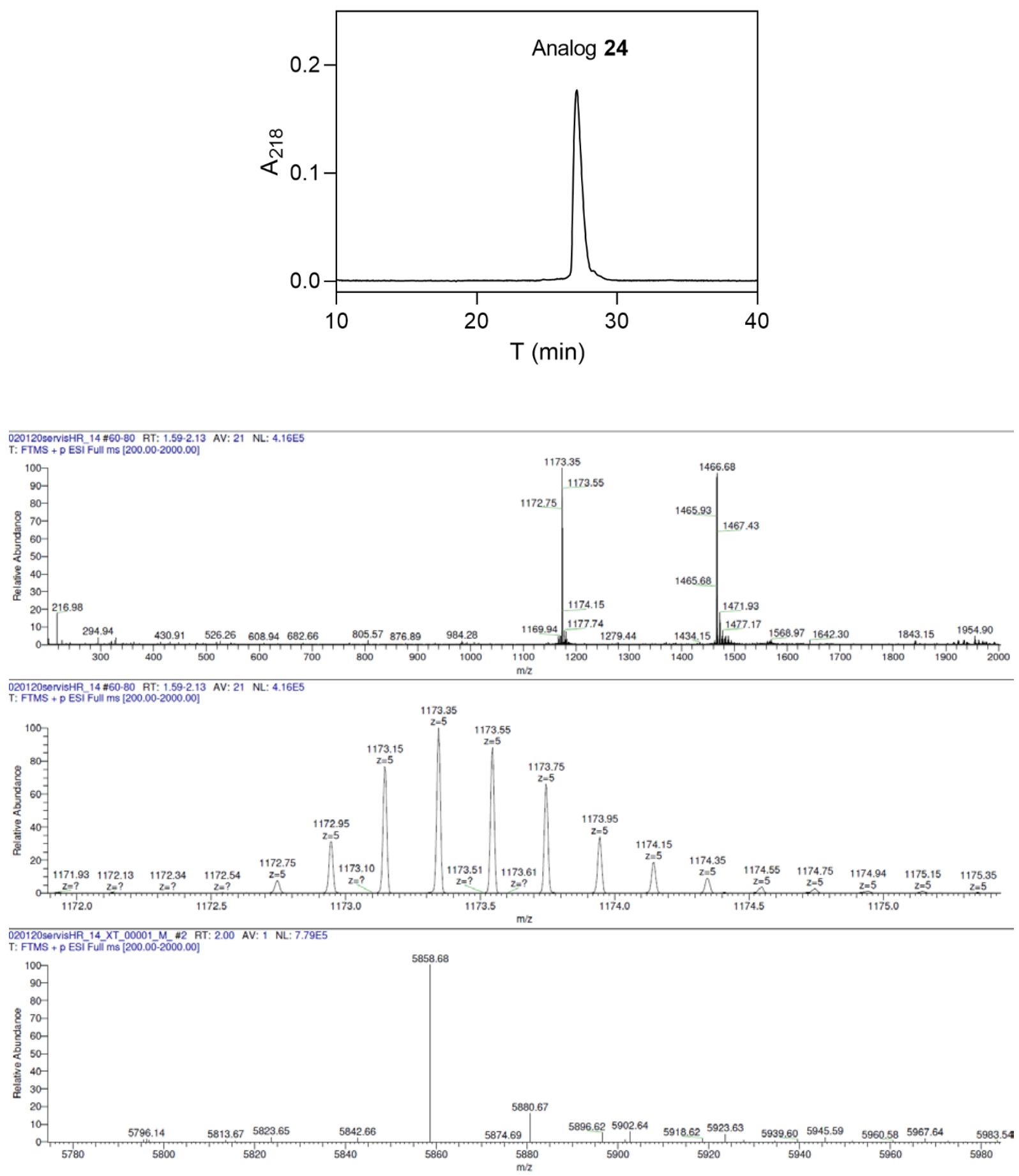

Figure S25. Upper panel: RP-HPLC analysis of analog 24 ( $\left[\mathrm{Ala}^{\mathrm{B} 29}, \mathrm{Ile}^{\mathrm{B} 31}\right.$, amide $\left.{ }^{\mathrm{B} 31}\right]$-insulin). Lower panel: HR-MS of analog 24, Mw (monoisotopic for $\mathrm{C}_{260} \mathrm{H}_{388} \mathrm{~N}_{66} \mathrm{O}_{77} \mathrm{~S}_{6}$ ) calculated 5858.6798, Mw found 5858.68. 

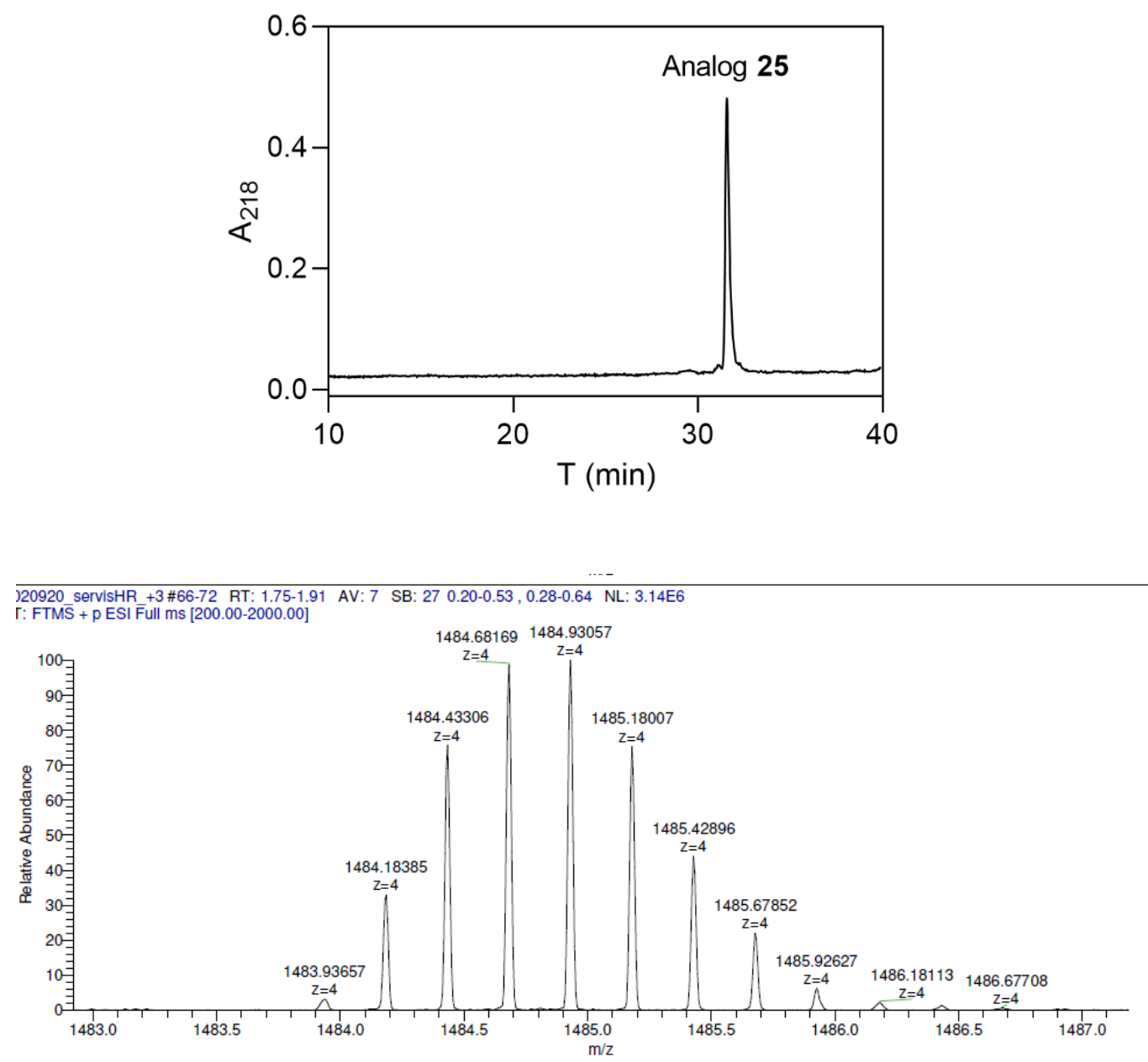

20920 servisHR+3 XT_00001_M \#2 RT: 2.00 AV: 1 SB: 2 1.00, 1.00 NL: 4.58E6

Г: FTMS + p ESI Full ms [200.00-2000.00]
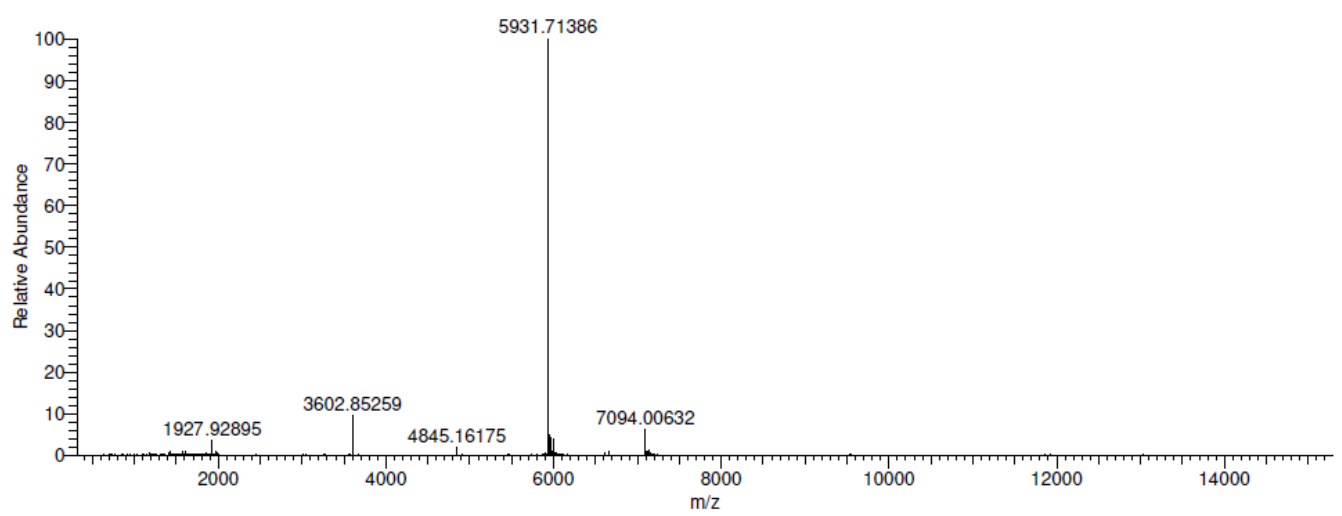

Figure S26. Upper panel: RP-HPLC analysis of analog 25 ([Ala ${ }^{\mathrm{B} 29}, \operatorname{Trp}^{\mathrm{B} 31}$, amide $\left.{ }^{\mathrm{B} 31}\right]$-insulin). Lower panel: HR-MS of analog 25, Mw (monoisotopic for $\mathrm{C}_{265} \mathrm{H}_{387} \mathrm{~N}_{67} \mathrm{O}_{77} \mathrm{~S}_{6}$ ) calculated 5931.6751, Mw found 5931.7139. 

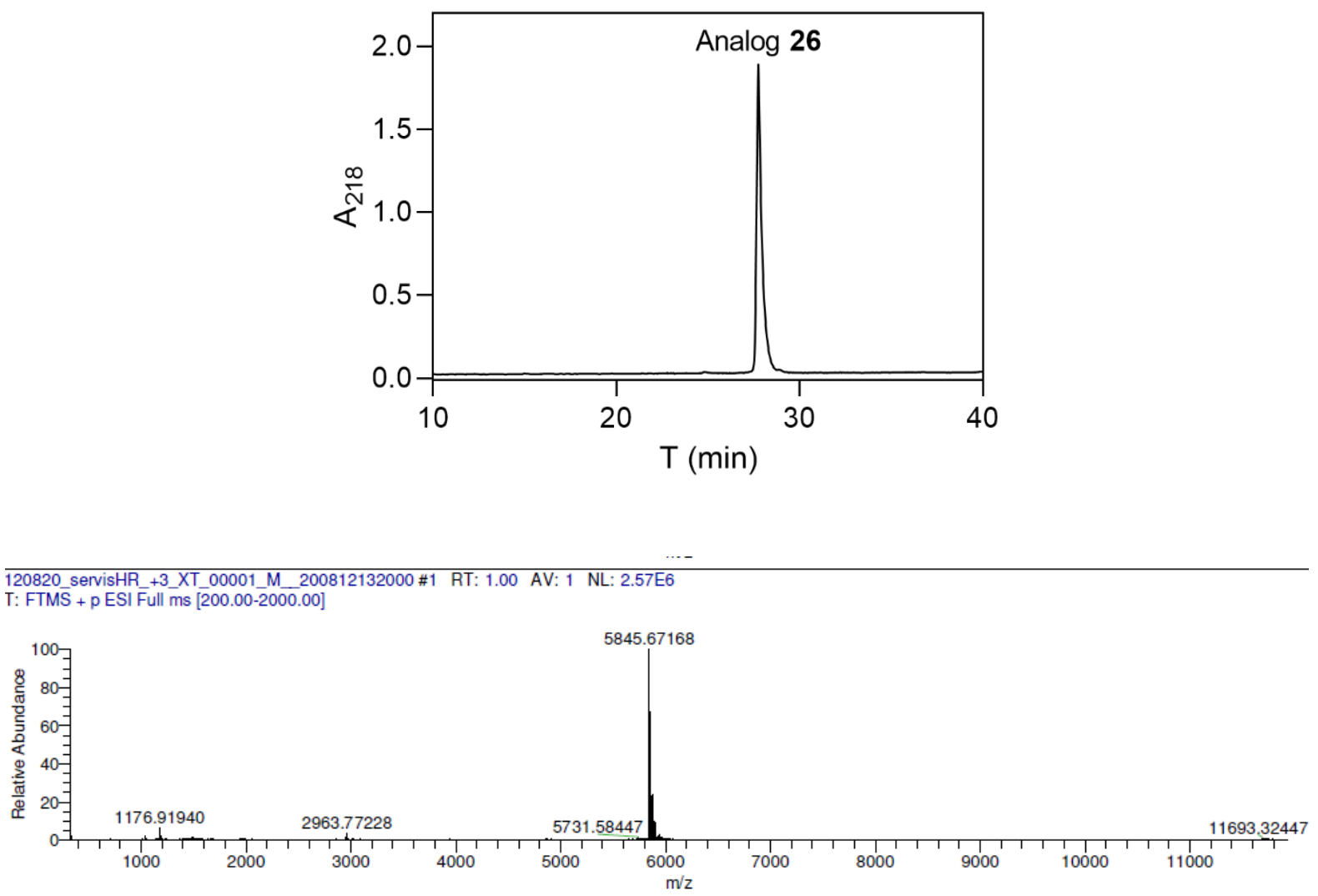

120820 servisHR_+3_XT_00001_M_200812132000 \#1 RT: 1.00 AV: 1 NL: $2.57 E 6$

T: FTMS + p ESI Full ms [200.00-2000.00]

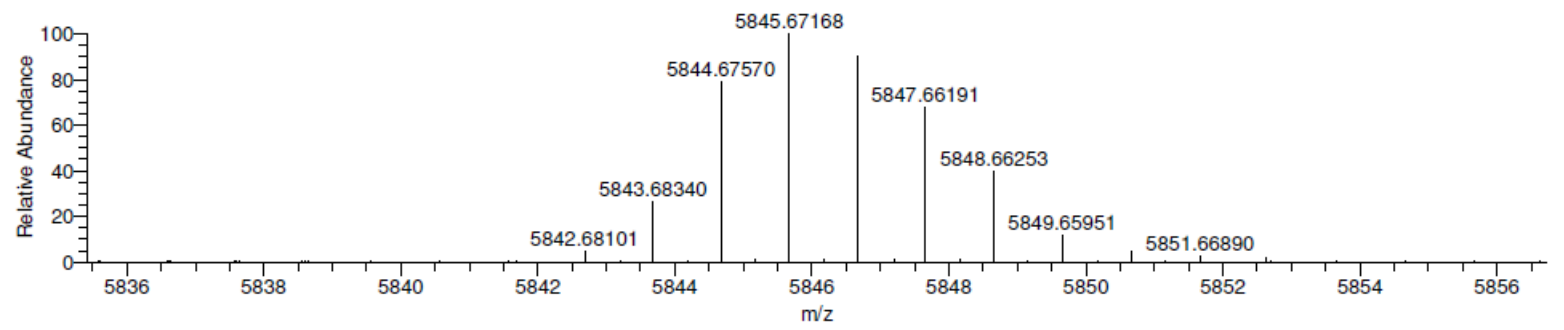

Figure S27. Upper panel: RP-HPLC analysis of analog 26 ([Ala ${ }^{\mathrm{B} 29}, \mathrm{Pro}^{\mathrm{B} 31}$, amide $\left.{ }^{\mathrm{B} 31}\right]$-insulin). Lower panel: HR-MS of analog 26, Mw (monoisotopic for $\mathrm{C}_{259} \mathrm{H}_{384} \mathrm{~N}_{66} \mathrm{O}_{77} \mathrm{~S}_{6}$ ) calculated 5842.6485, Mw found 5842.6810. 

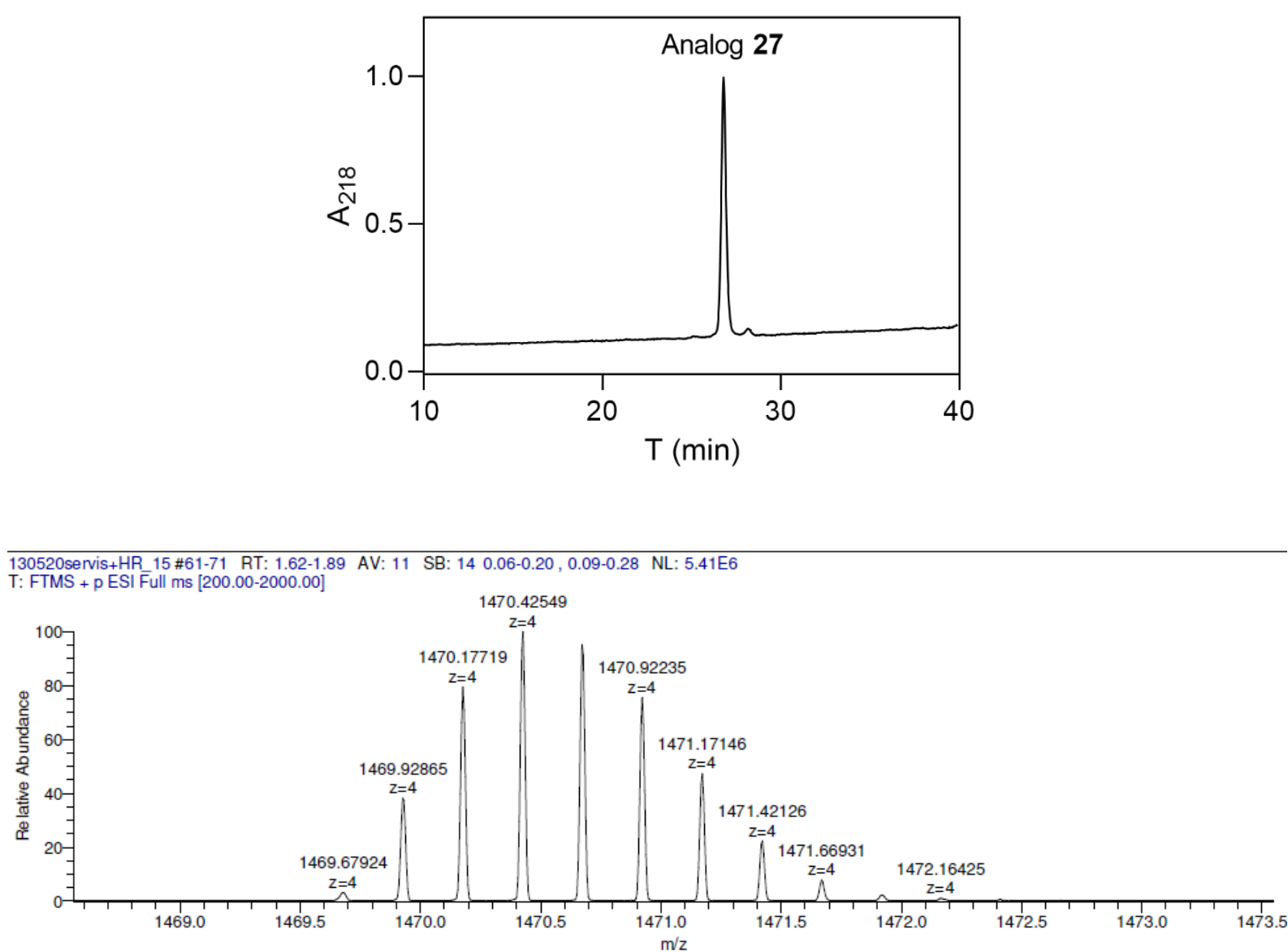

130520servis+HR_15_XT_00001_M_\#2 RT: 2.00 AV: 1 SB: 2 1.00, 1.00 NL: 1.04E7

T: FTMS + p ESI Full ms [200.00-2000.00]

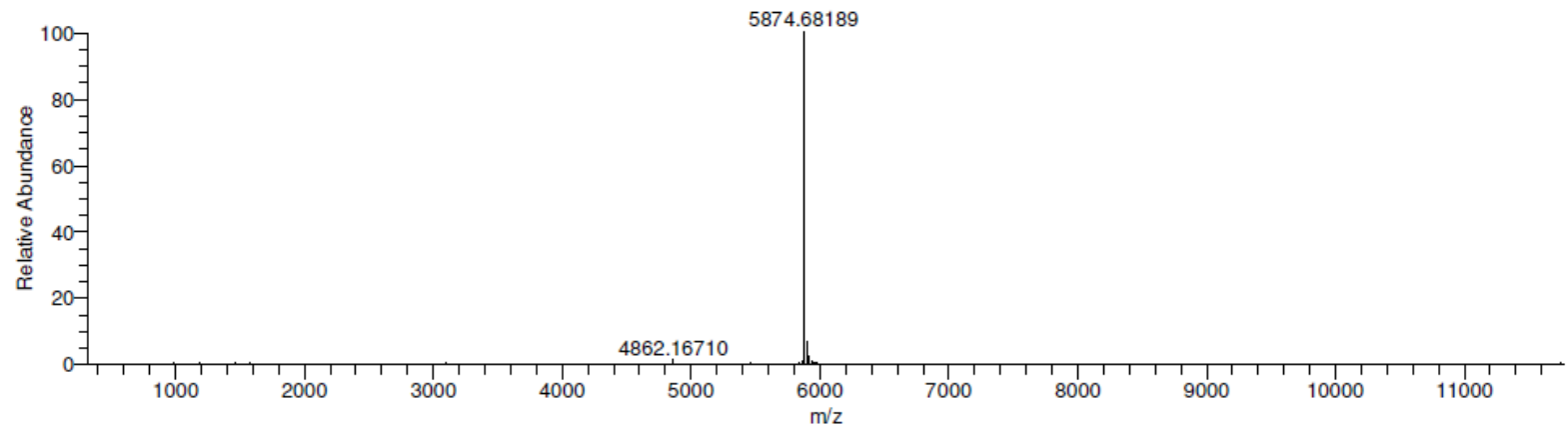

Figure S28. Upper panel: RP-HPLC analysis of analog 27 ([Ala ${ }^{\mathrm{B} 29}, D-\mathrm{Glu}^{\mathrm{B} 31}$, amide $\left.{ }^{\mathrm{B} 31}\right]$-insulin). Lower panel: HR-MS of analog 27, Mw (monoisotopic for $\mathrm{C}_{259} \mathrm{H}_{384} \mathrm{~N}_{66} \mathrm{O}_{79} \mathrm{~S}_{6}$ ) calculated 5874.6384, Mw found 5842.6819. 

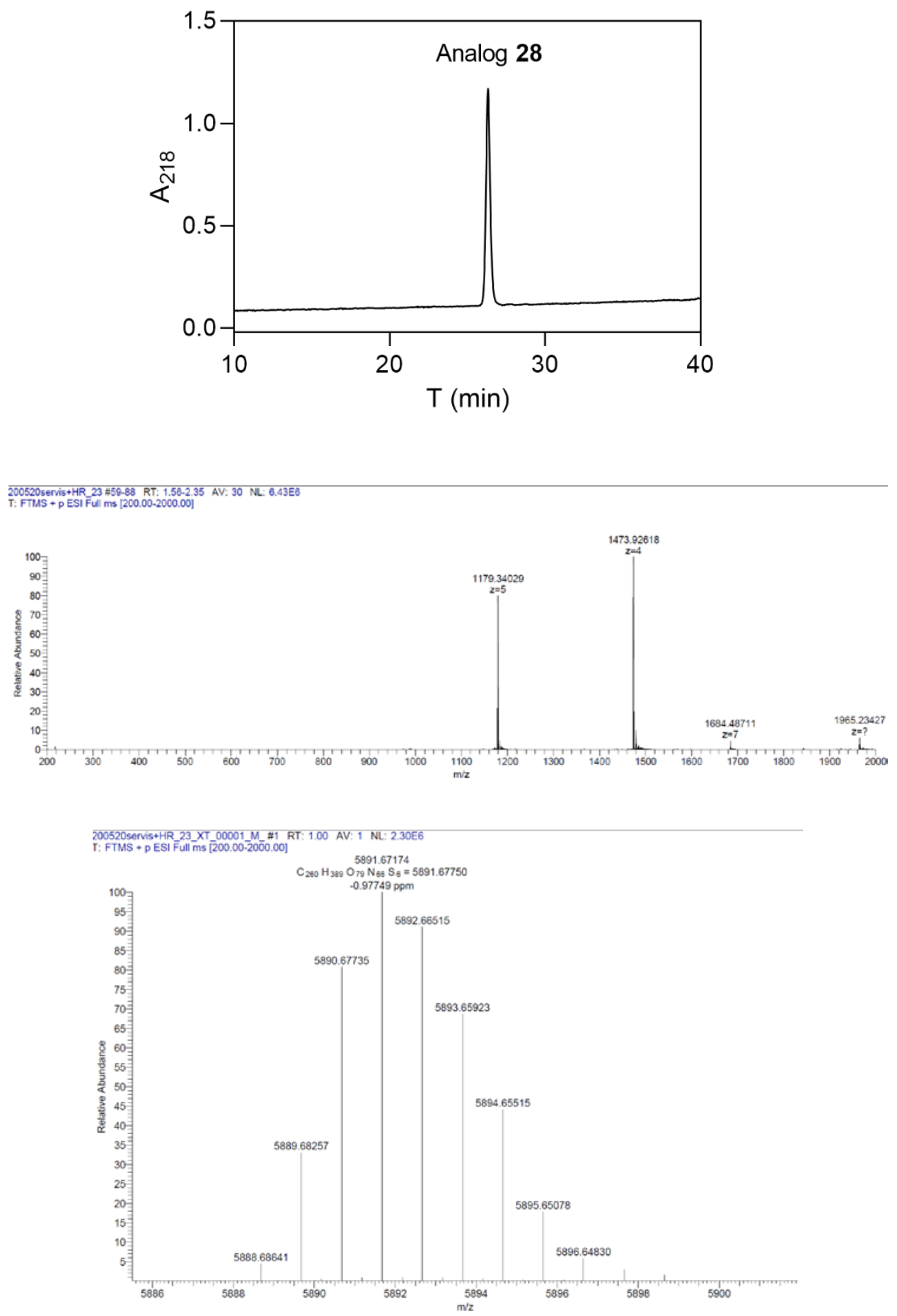

Figure S29. Upper panel: RP-HPLC analysis of analog 28 ([Ala ${ }^{\mathrm{B} 29}, \mathrm{hGlu}^{\mathrm{B} 31}$, amide $\left.{ }^{\mathrm{B} 31}\right]$-insulin). Lower panel: HR-MS of analog 28, Mw (monoisotopic for $\mathrm{C}_{260} \mathrm{H}_{386} \mathrm{~N}_{66} \mathrm{O}_{79} \mathrm{~S}_{6}$ ) calculated 5888.6540, Mw found 5888.6864. 

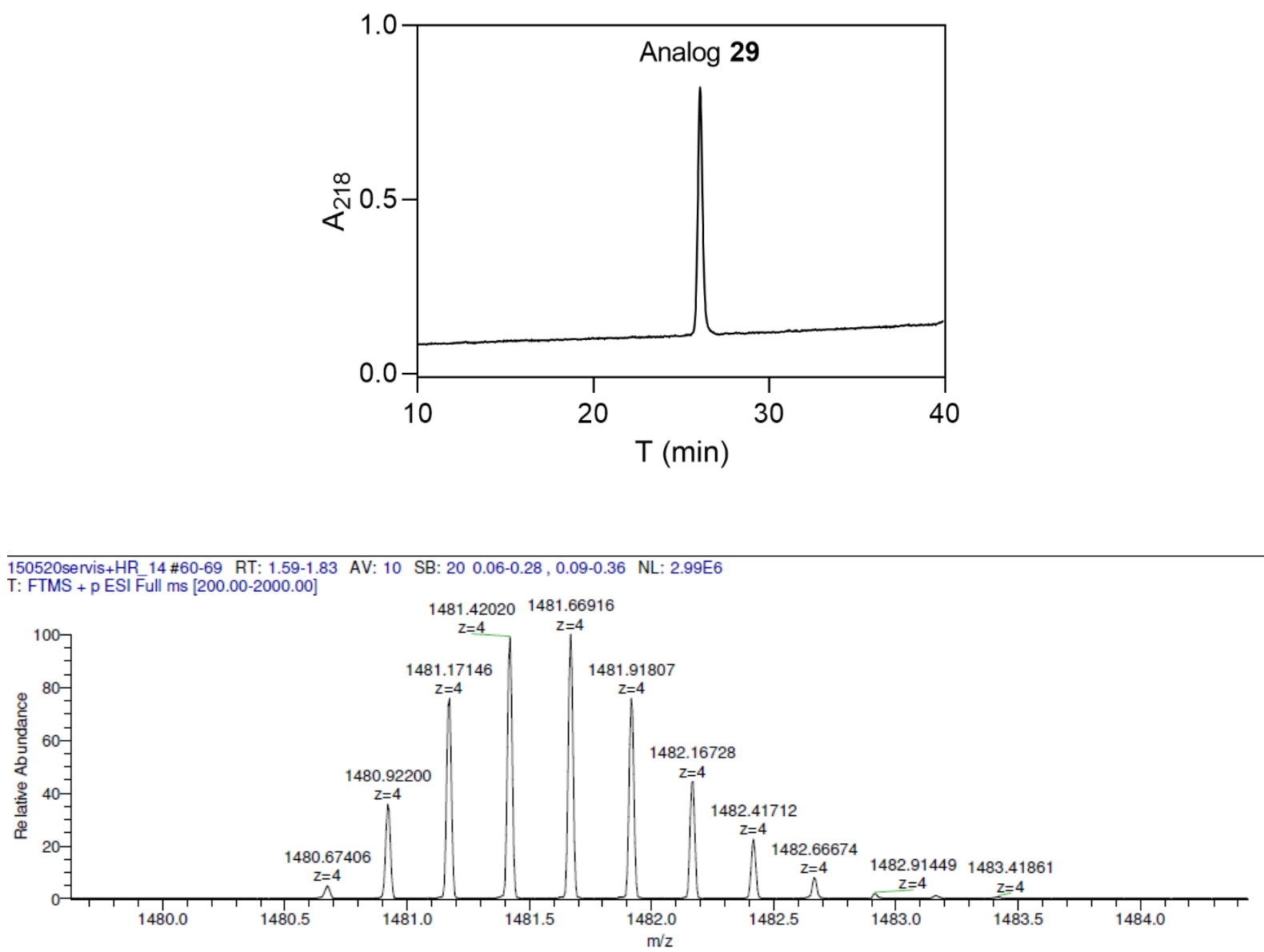

150520servis+HR_14_XT_00001_M_\#2 RT: 2.00 AV: 1 SB: 2 1.00, 1.00 NL: 5.11E6

T: FTMS + p ESI Full ms [200.00-2000.00]

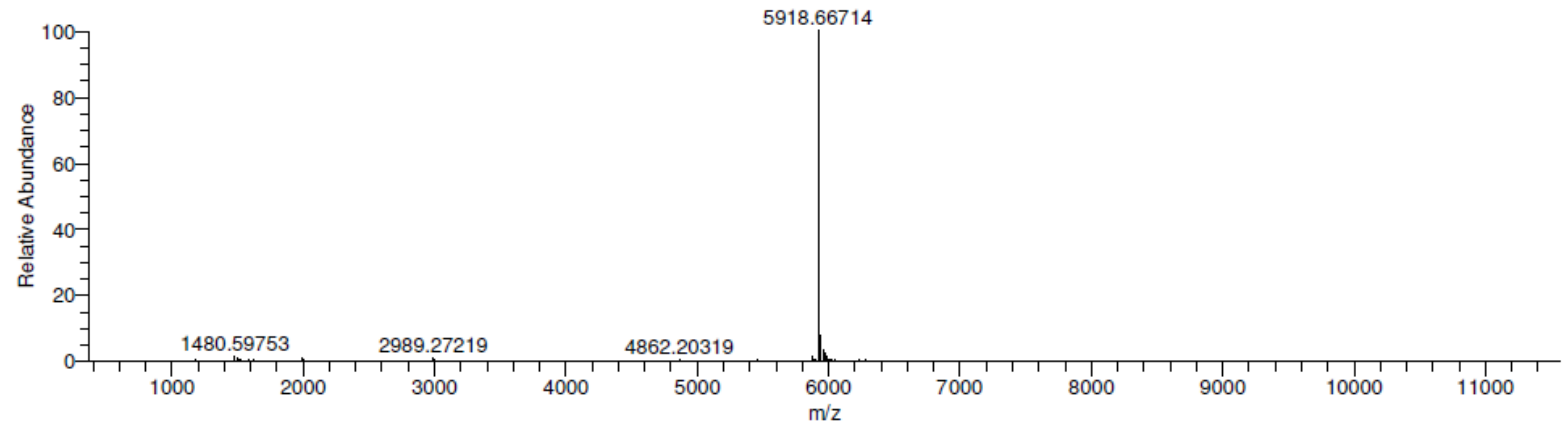

Figure S30. Upper panel: RP-HPLC analysis of analog 29 ([Ala ${ }^{\mathrm{B} 29}, \mathrm{Gla}^{\mathrm{B} 31}$, amide $\left.{ }^{\mathrm{B} 31}\right]$-insulin). Lower panel: HR-MS of analog 29, Mw (monoisotopic for $\mathrm{C}_{260} \mathrm{H}_{384} \mathrm{~N}_{66} \mathrm{O}_{81} \mathrm{~S}_{6}$ ) calculated 5918.6282, Mw found 5918.6671. 

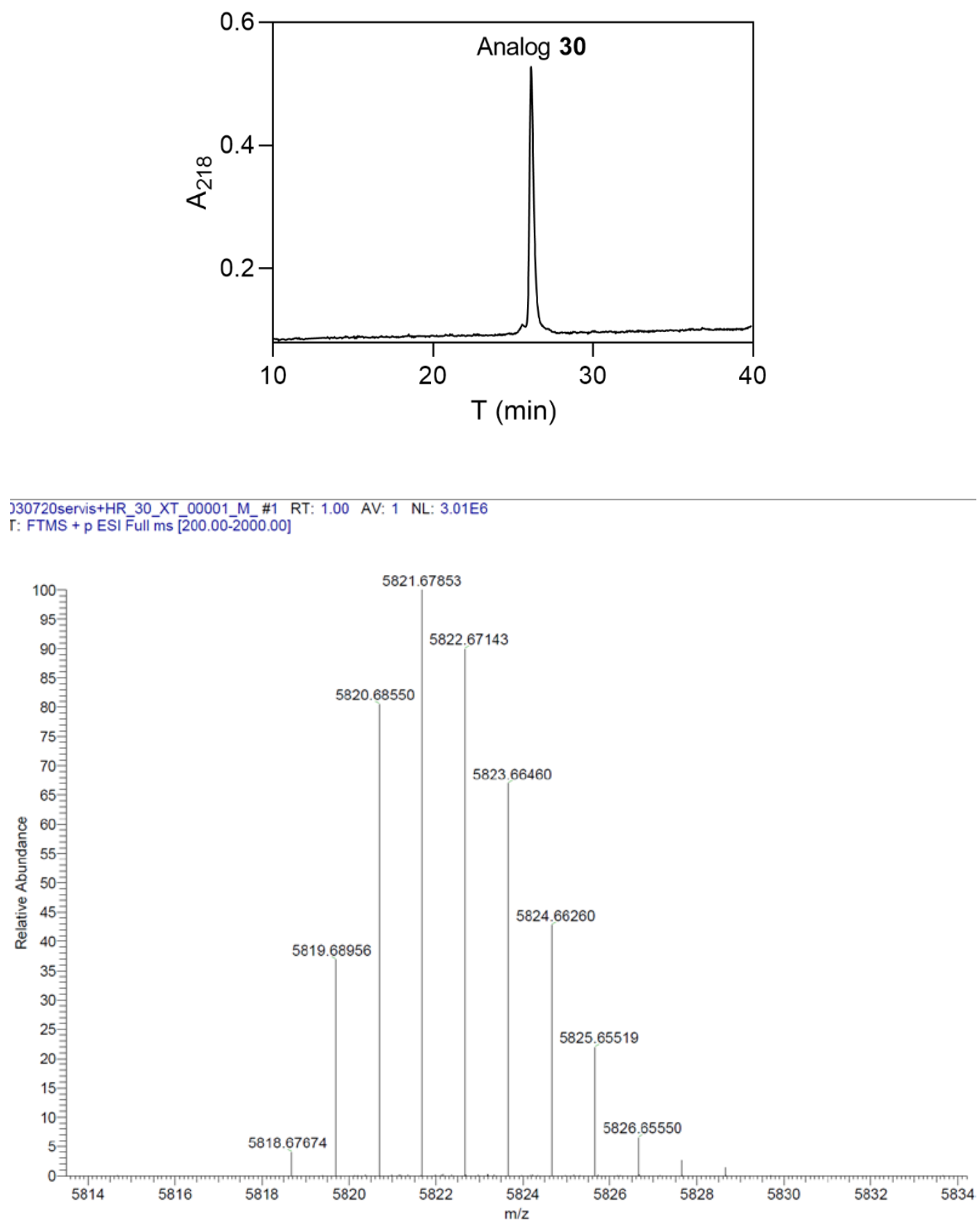

Figure S31. Upper panel: RP-HPLC analysis of analog 30 ([Glu $\left.{ }^{\mathrm{A} 18}\right]$-insulin). Lower panel: HRMS of analog 30, Mw (monoisotopic for $\mathrm{C}_{258} \mathrm{H}_{384} \mathrm{~N}_{64} \mathrm{O}_{78} \mathrm{~S}_{6}$ ) calculated 5818.6373, Mw found 5918.6667. 

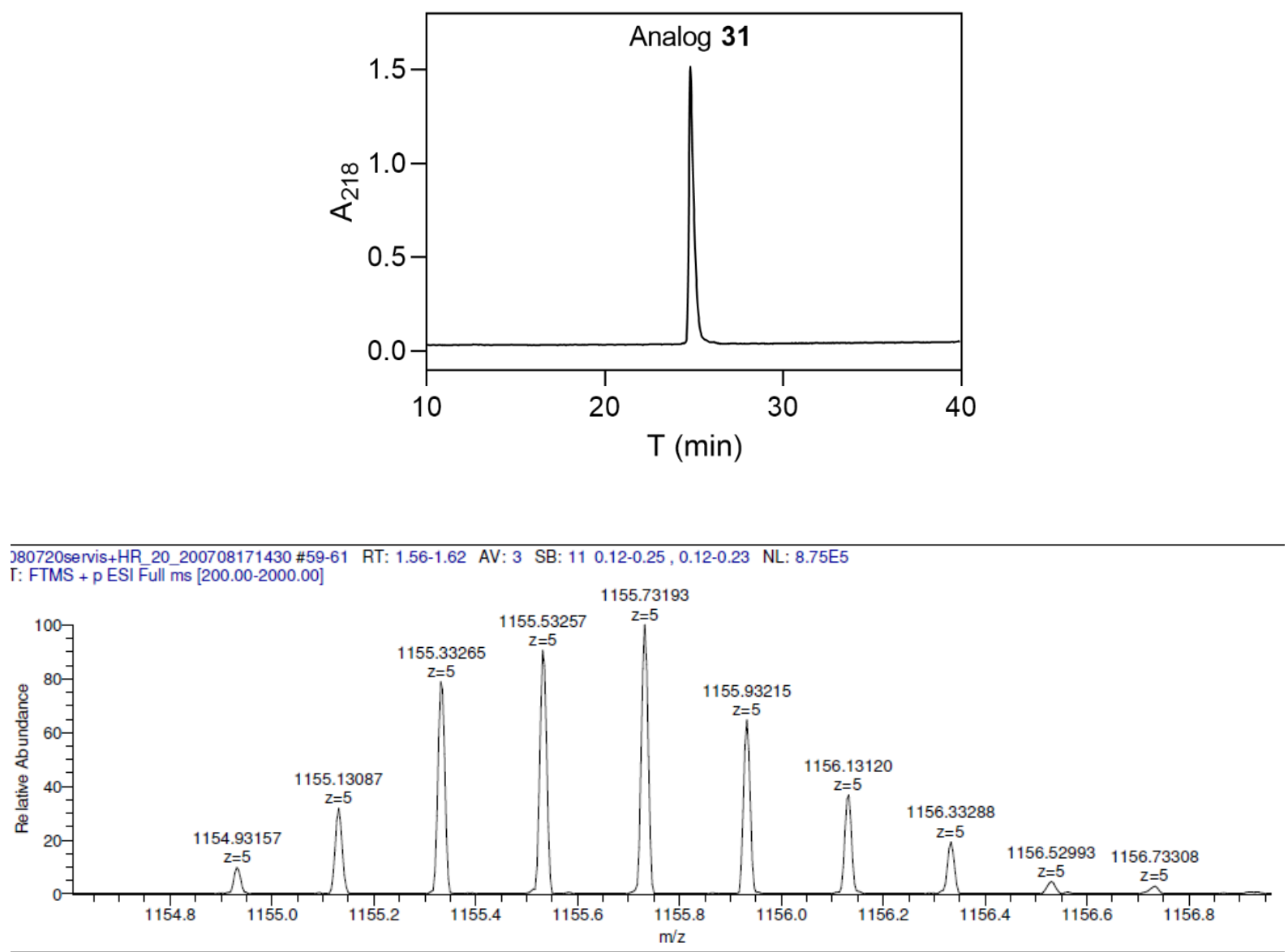

380720servis+HR_20_200708171430_XT_00001_M_\#2 RT: 2.00 AV: 1 SB: $21.00,1.00$ NL: 9.73E5

T: FTMS + p ESI Full ms [200.00-2000.00]

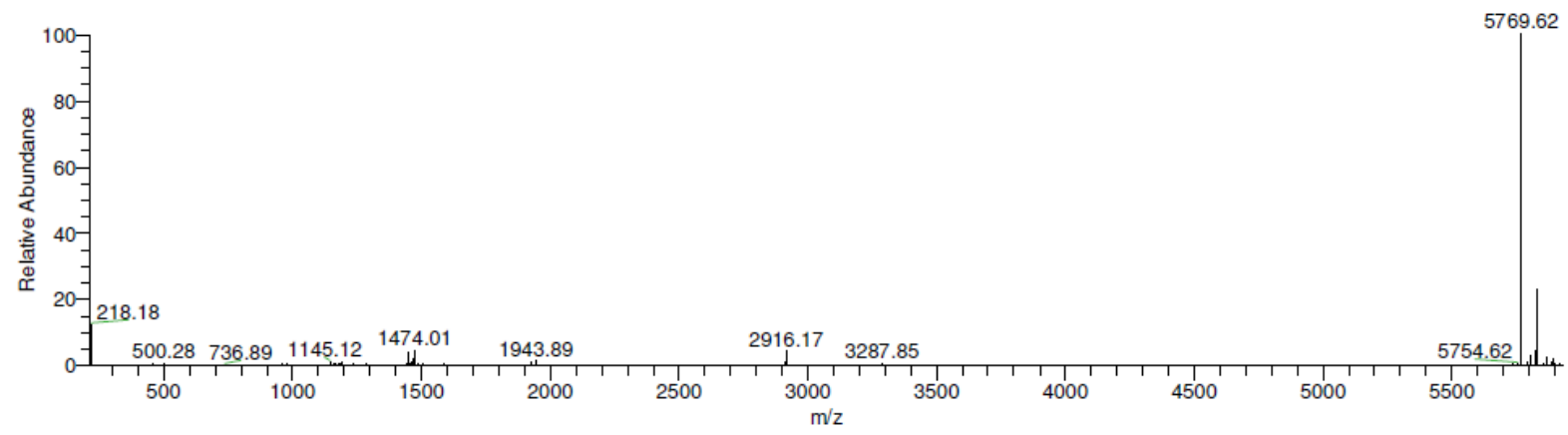

Figure S32. Upper panel: RP-HPLC analysis of analog 31 ([Glu $\left.{ }^{\mathrm{A} 14}\right]$-insulin). Lower panel: HRMS of analog 31, Mw (monoisotopic for $\mathrm{C}_{253} \mathrm{H}_{381} \mathrm{~N}_{65} \mathrm{O}_{78} \mathrm{~S}_{6}$ ) calculated 5769.6619, Mw found 5769.62. 

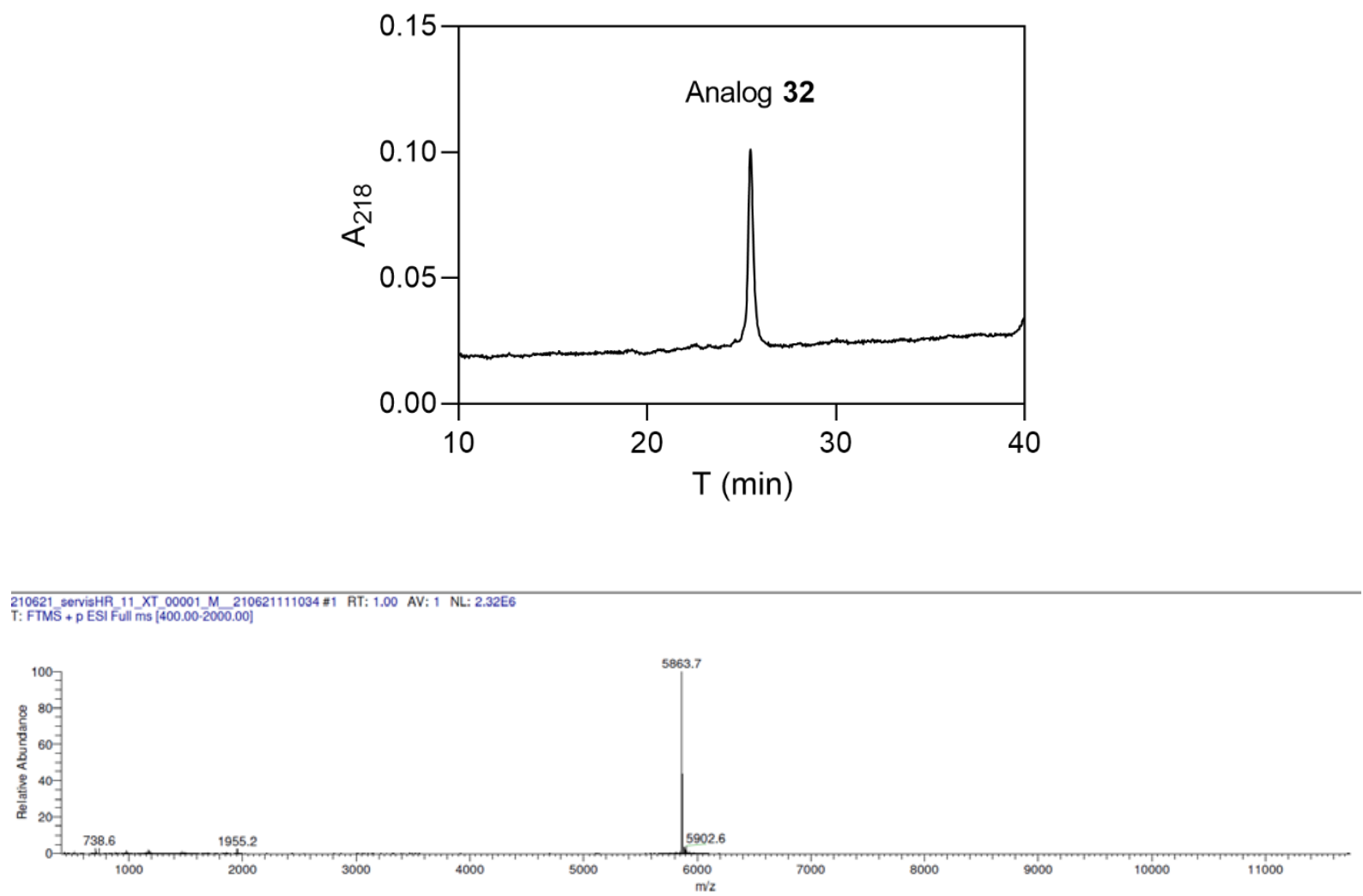

210621 servisHR 11_XT 00001 M 210621111034 \#1 RT: 1.00 AV: 1 NL: $2.32 E 6$

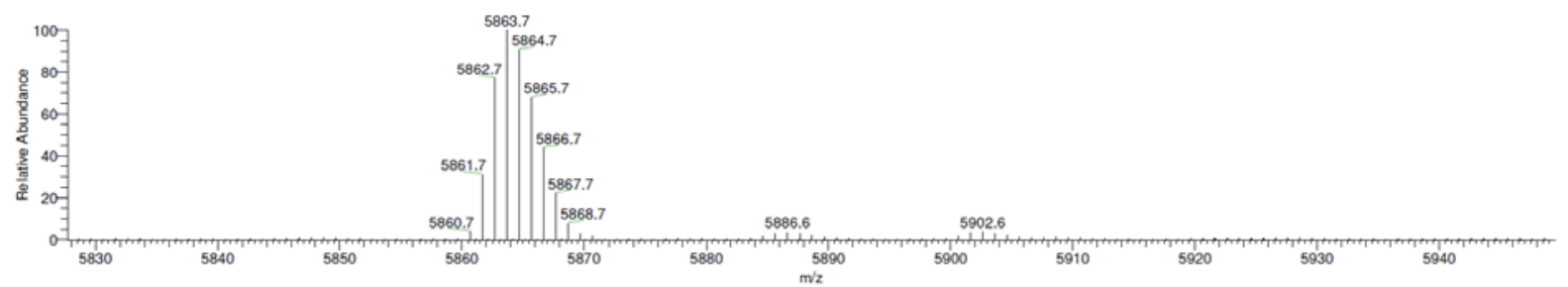

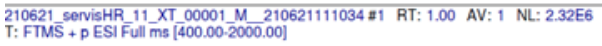

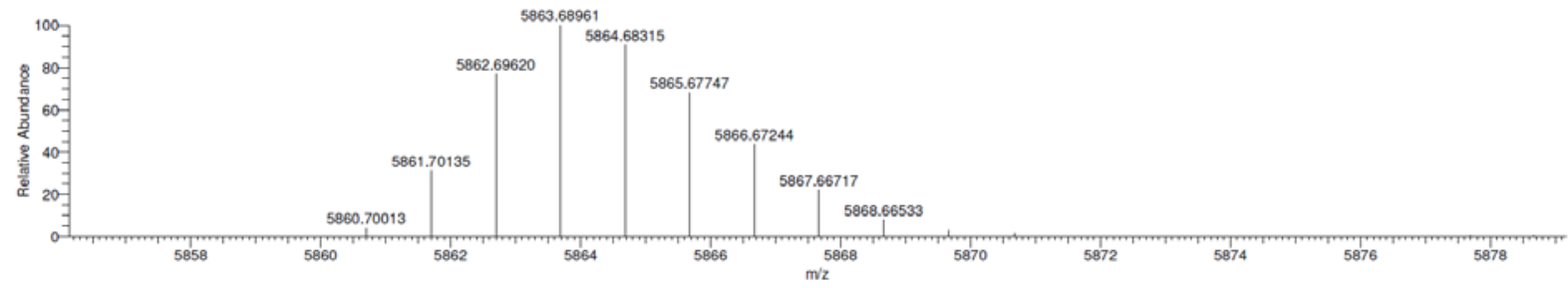

Figure S33. Upper panel: RP-HPLC analysis of analog 32 ([Gly $\left.{ }^{\mathrm{A}-1}\right]$-insulin). Lower panel: HRMS of analog 32, Mw (monoisotopic for $\mathrm{C}_{259} \mathrm{H}_{386} \mathrm{~N}_{66} \mathrm{O}_{78} \mathrm{~S}_{6}$ ) calculated 5860.6591, Mw found 5860.7001 . 

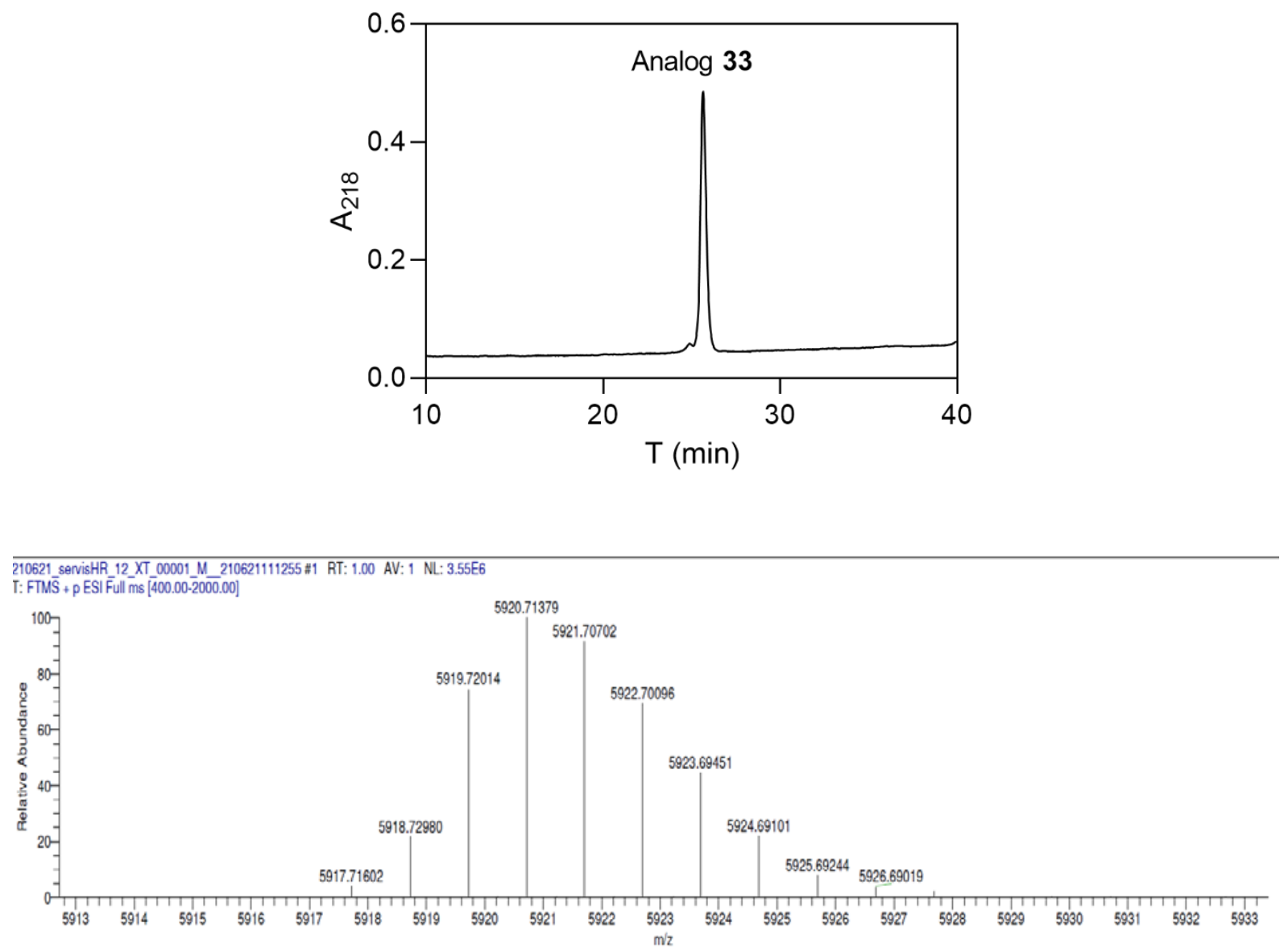

Figure S34. Upper panel: RP-HPLC analysis of analog 33 ([Gly ${ }^{\mathrm{A}-2}$, Gly $\left.{ }^{\mathrm{A}-1}\right]$-insulin). Lower panel: HR-MS of analog 33, Mw (monoisotopic for $\mathrm{C}_{261} \mathrm{H}_{389} \mathrm{~N}_{67} \mathrm{O}_{79} \mathrm{~S}_{6}$ ) calculated 5917.6806, Mw found 5917.7160 . 


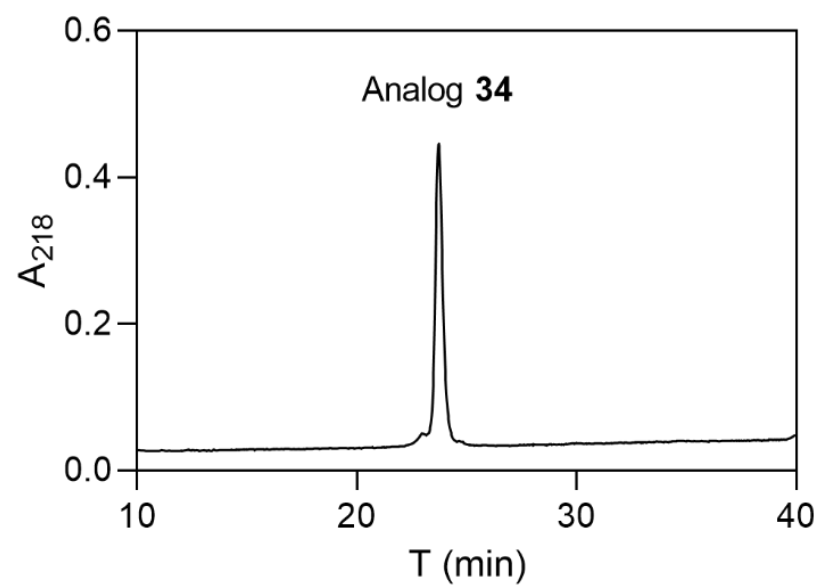

210621 servisHR 13 XT_00001_M_210621111433 \#1 RT: 1.00 AV: 1 NL: 3.13E6
T:FTMS + p ESI Full ms $[400.00-2000.00]$

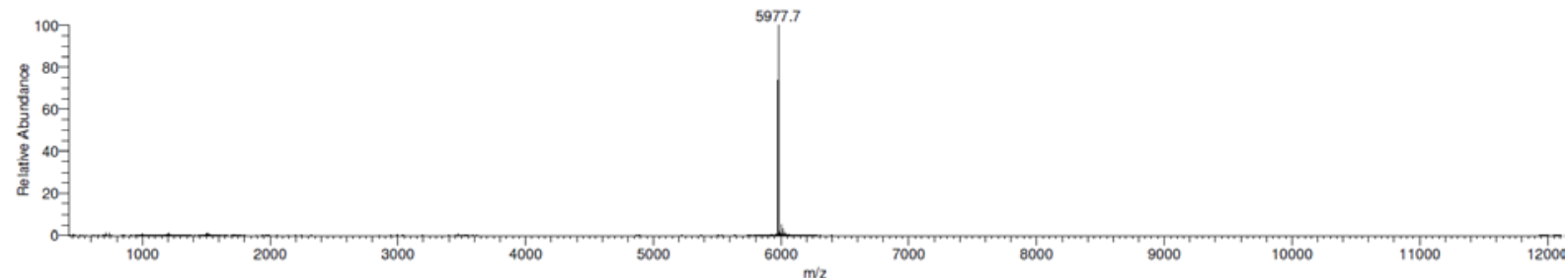

210621 servisHR 13 XT $000011 \mathrm{M} 210621111433 \approx 1$ RT: 1.00 AV: 1 NL: 3.13E6
T: FTMS + P ESI Full ms $[400.00-2000.00]$

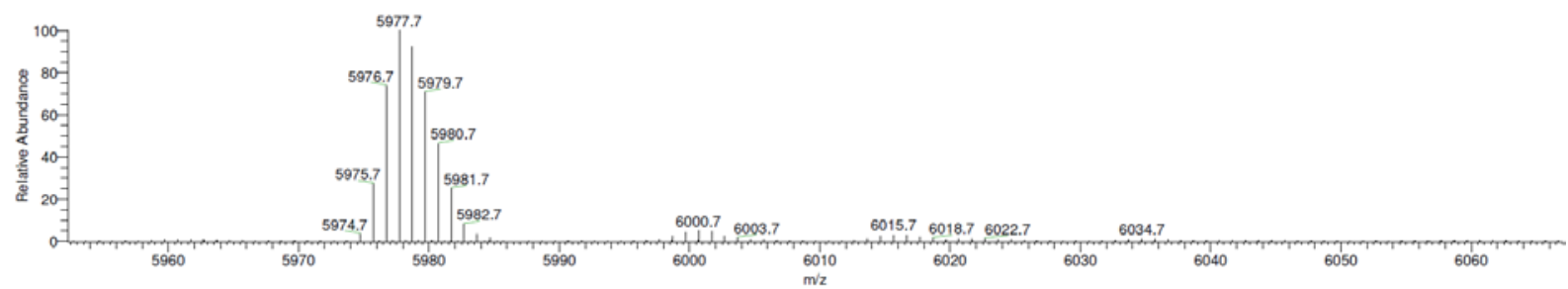

210621 servisHR_13_XT_00001_M 210621111433 \#1 RT: 1.00 AV: 1 NL: 3.13E6
T: FTMS + P ESIFull ms [400.00-2000.00]

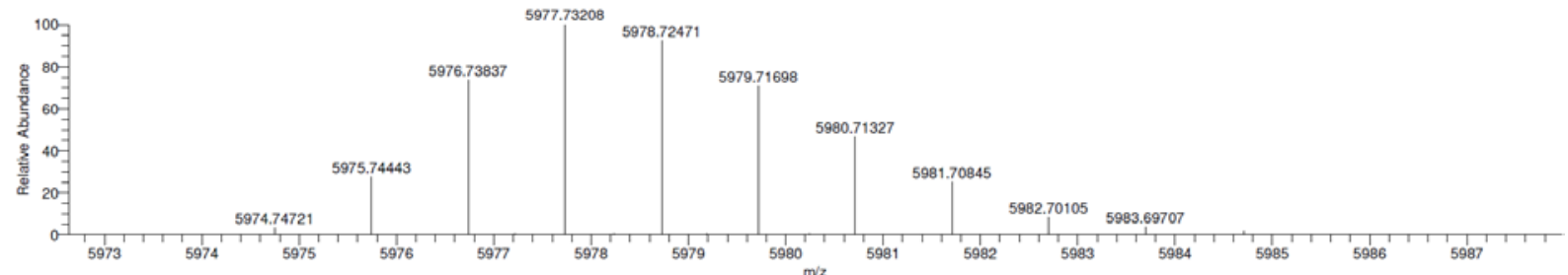

Figure S35. Upper panel: RP-HPLC analysis of analog 34 ([Gly ${ }^{\mathrm{A}-3}$, Gly $\left.{ }^{\mathrm{A}-2}, \mathrm{Gly}^{\mathrm{A}-1}\right]$-insulin). Lower panel: HR-MS of analog 34, Mw (monoisotopic for $\mathrm{C}_{263} \mathrm{H}_{392} \mathrm{~N}_{68} \mathrm{O}_{80} \mathrm{~S}_{6}$ ) calculated 5974.7020, Mw found 5974.7472 . 

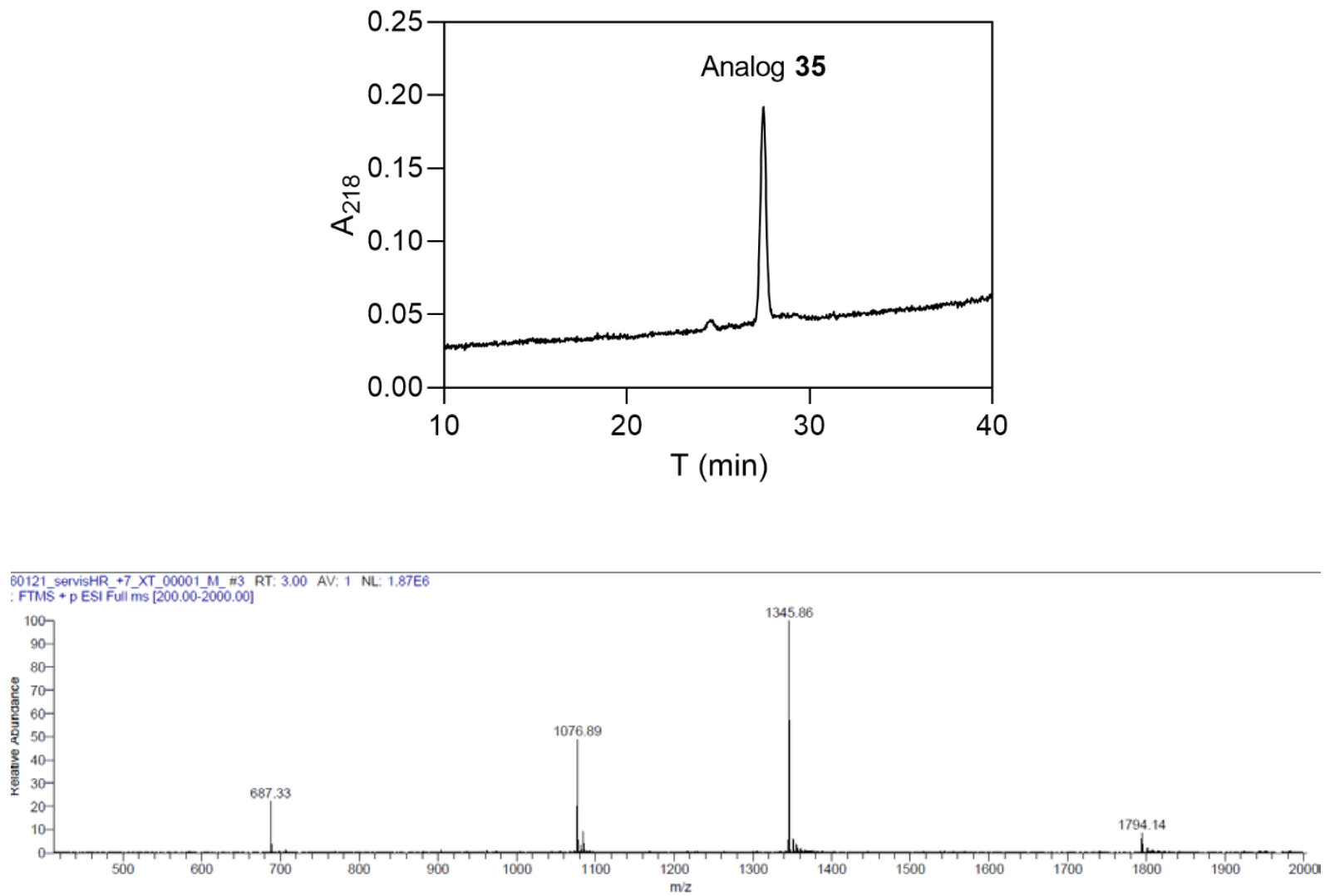

60121 servisHR +7_XT_00001_M_ H1 RT: 1.00 AV: 1 NL: 654 E5
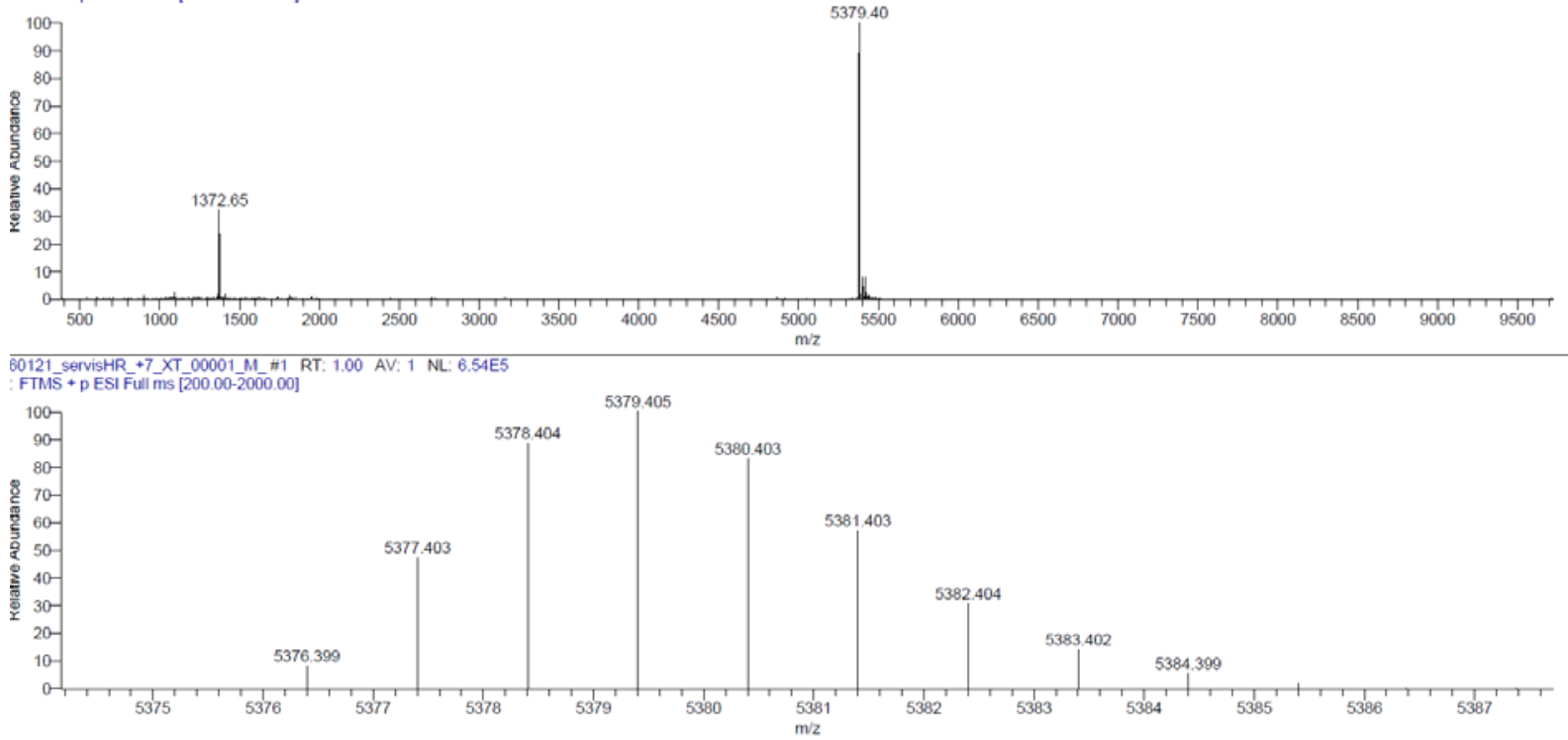

Figure S36. Upper panel: RP-HPLC analysis of analog 35 ([des(tetrapeptide $\left.\left.{ }^{\mathrm{B} 27-\mathrm{B} 30}\right)\right]$-insulin). Lower panel: HR-MS of analog 35, Mw (monoisotopic for $\mathrm{C}_{238} \mathrm{H}_{350} \mathrm{~N}_{60} \mathrm{O}_{71} \mathrm{~S}_{6}$ ) calculated 5376.3946, Mw found 5376.3990. 

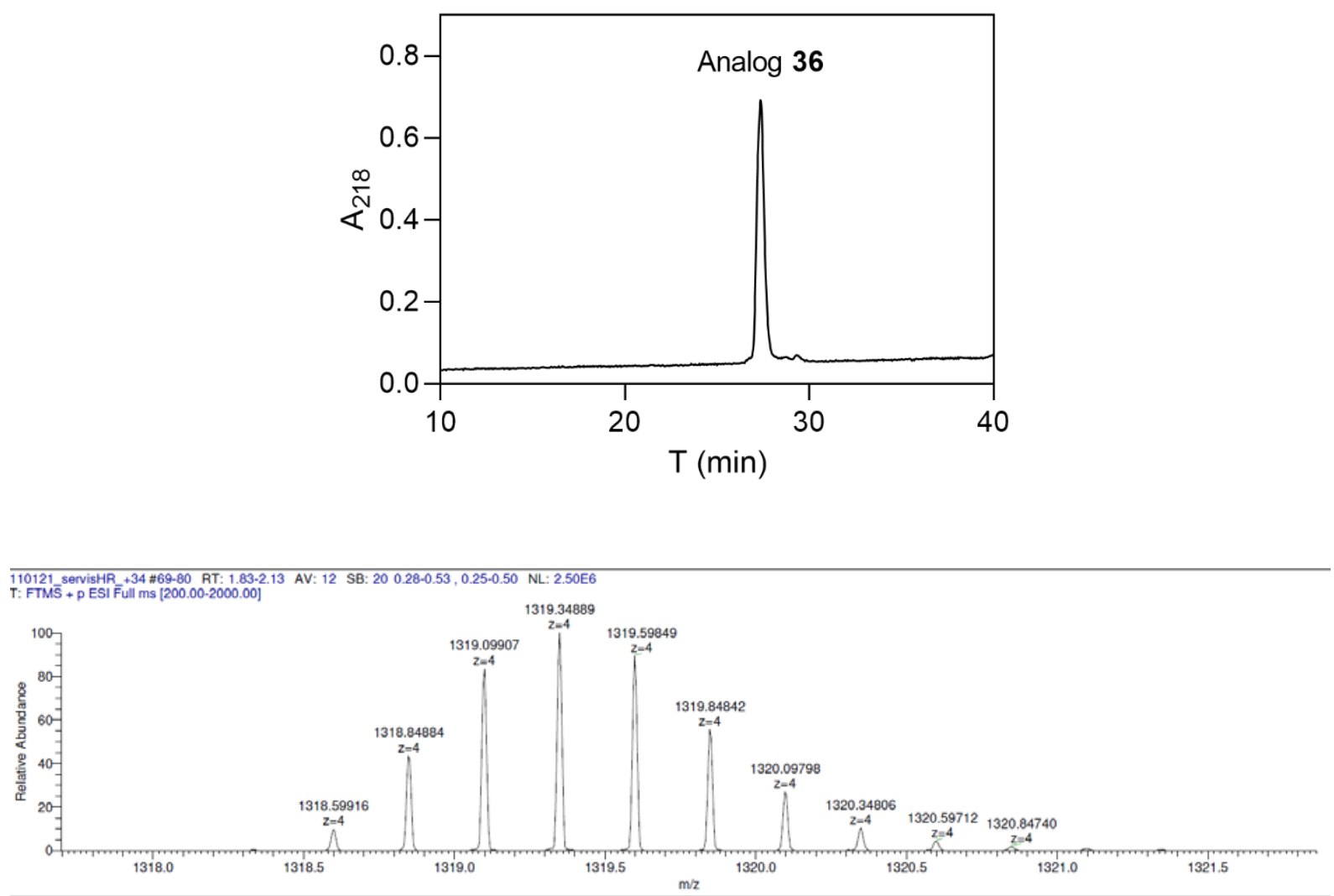

110121 servisHR_34_XT_00001_M_\#2 RT: 2.00 AV: 1 SB: $21.00,1.00$ NL: $3.26 E 6$
T: FTMS + PESI Full ms $[200.00-2000$.00]

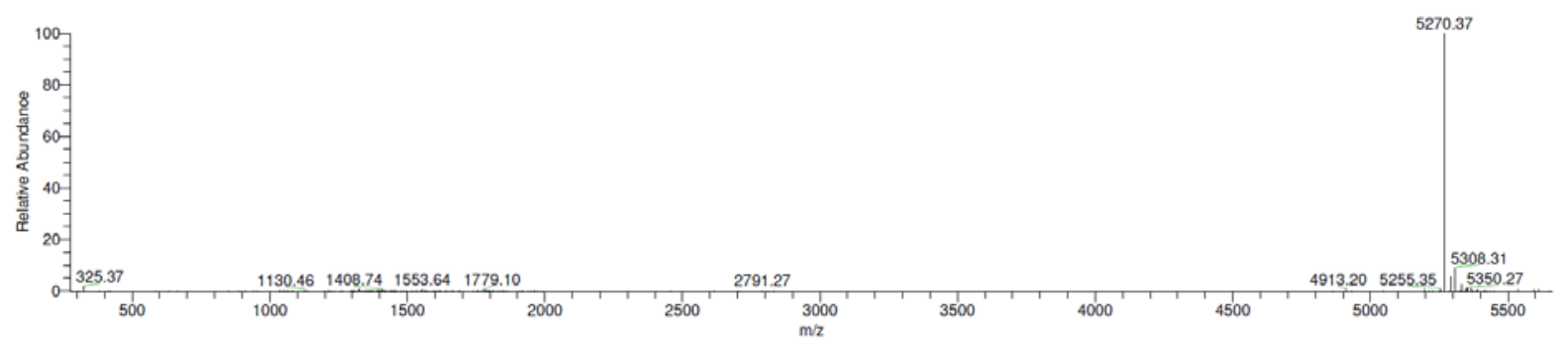

Figure S37. Upper panel: RP-HPLC analysis of analog 36 ([des(tetrapeptide ${ }^{\mathrm{B} 27-\mathrm{B} 30}$ ), Gly $\left.{ }^{\mathrm{B} 26}\right]$ insulin). Lower panel: HR-MS of analog 36, Mw (monoisotopic for $\mathrm{C}_{231} \mathrm{H}_{344} \mathrm{~N}_{60} \mathrm{O}_{70} \mathrm{~S}_{6}$ ) calculated 5270.3527, Mw found 5270.3700. 

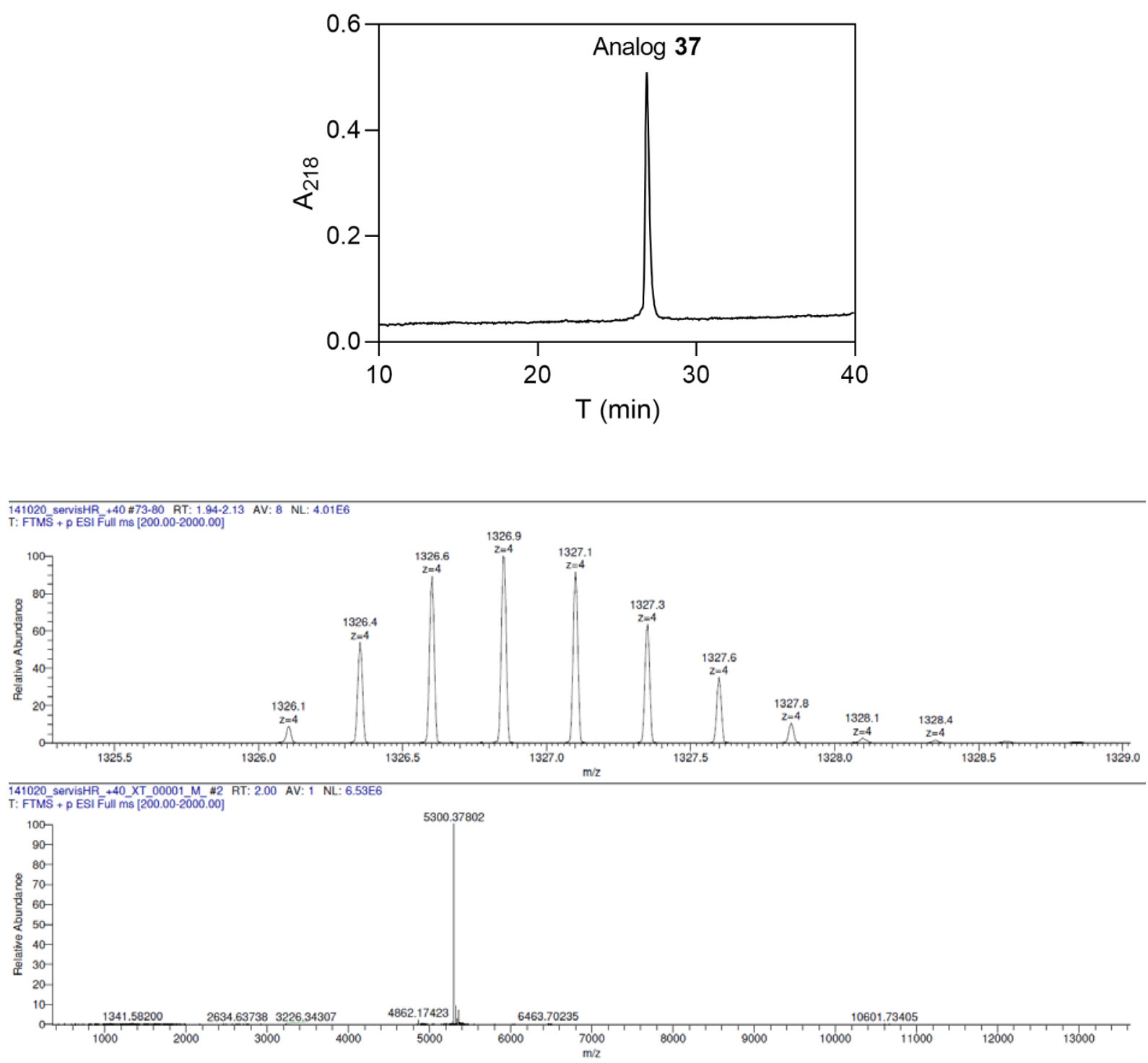

Figure S38. Upper panel: RP-HPLC analysis of analog 37 ([des(tetrapeptide ${ }^{\mathrm{B} 27-\mathrm{B} 30}$ ), Ser $\left.^{\mathrm{B} 26}\right]$ insulin). Lower panel: HR-MS of analog 37, Mw (monoisotopic for $\mathrm{C}_{232} \mathrm{H}_{346} \mathrm{~N}_{60} \mathrm{O}_{71} \mathrm{~S}_{6}$ ) calculated 5300.3633, Mw found 5300.3780. 

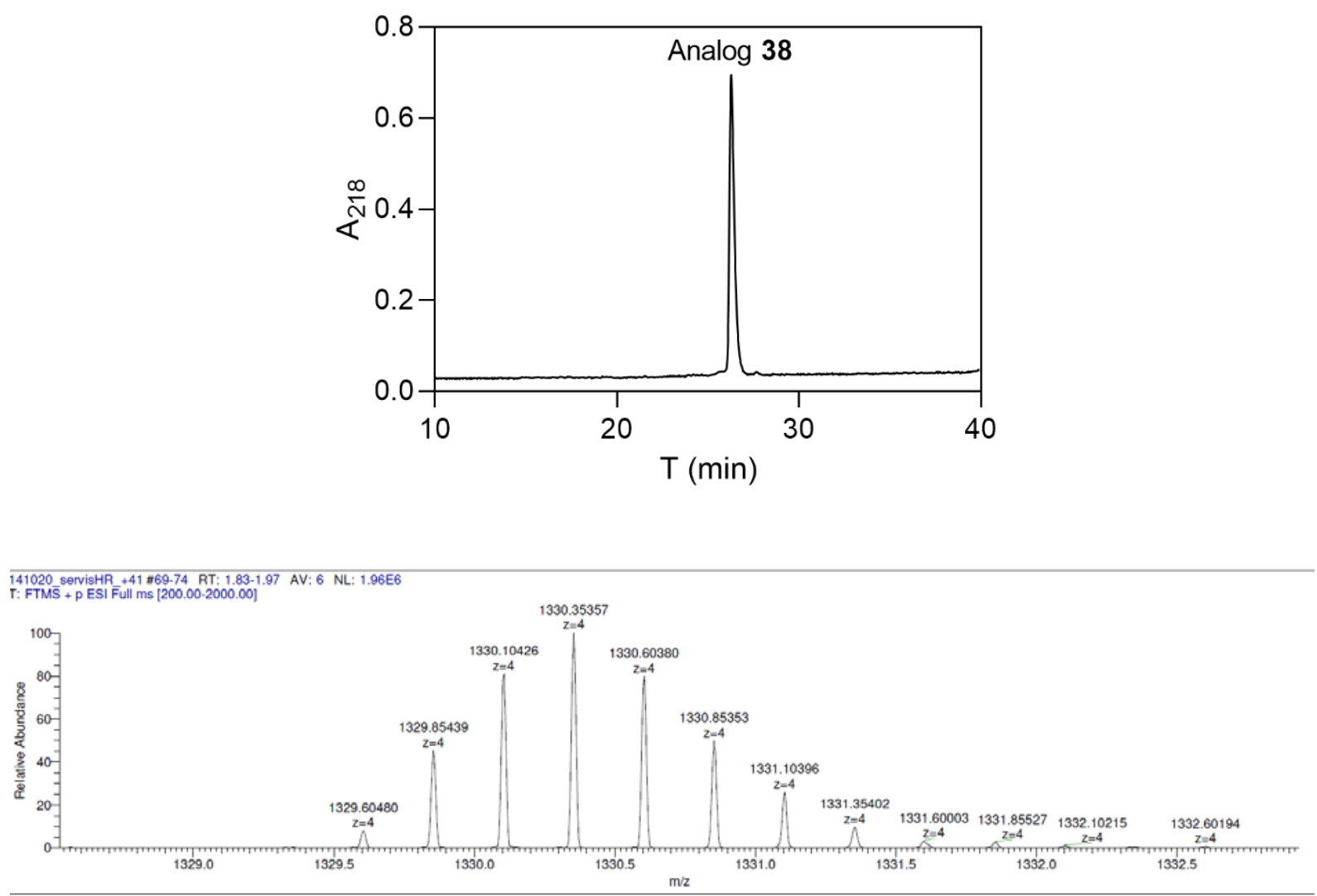

141020 servisHR_+41_XT_00001_M_\#2 RT: 2.00 AV: 1 NL: $2.91 \mathrm{EE}$
T: FTMS + p ESI Full ms $[200.00-2000.00]$

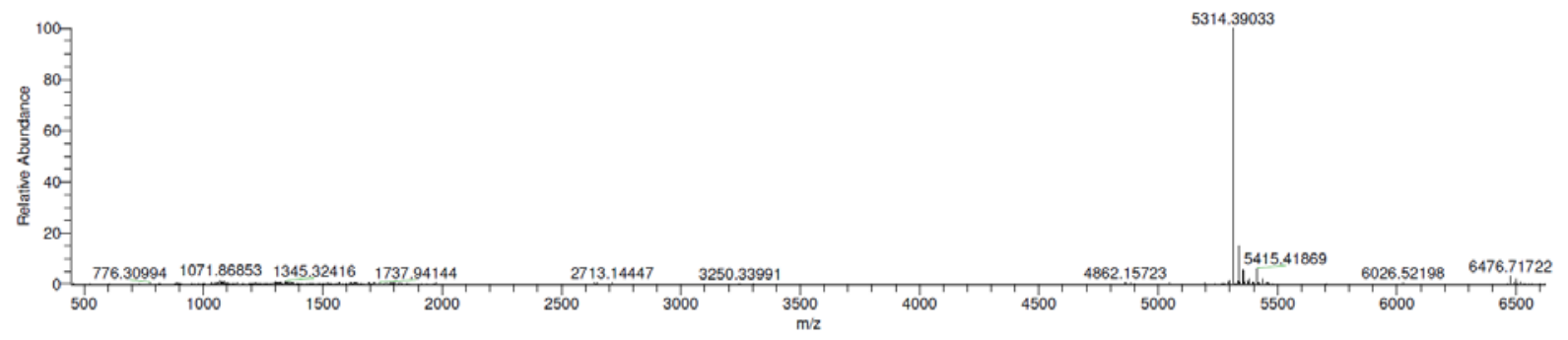

Figure S39. Upper panel: RP-HPLC analysis of analog 38 ([des (tetrapeptide ${ }^{\mathrm{B} 27-\mathrm{B} 30}$ ), $\left.\mathrm{Thr}^{\mathrm{B} 26}\right]$ insulin). Lower panel: HR-MS of analog 38, Mw (monoisotopic for $\mathrm{C}_{233} \mathrm{H}_{348} \mathrm{~N}_{60} \mathrm{O}_{71} \mathrm{~S}_{6}$ ) calculated 5314.3789, Mw found 5314.3903. 

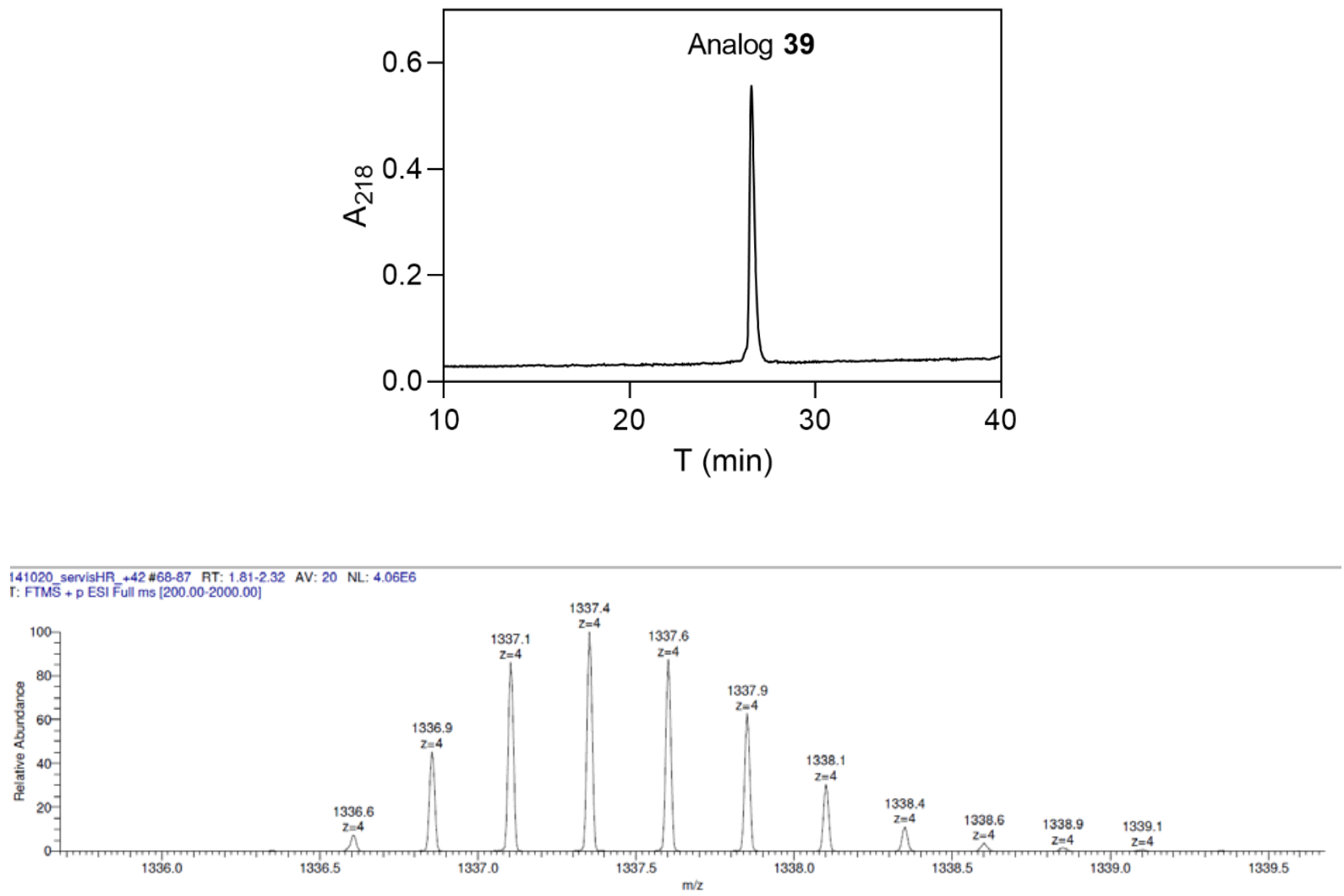

141020 sorvisHR_+42 XT_00001_M_\#2 RT: 2.00 AV: 1 NL: 6.58E6 r: FTMS + p ESI Full ms [200.00-2000.00

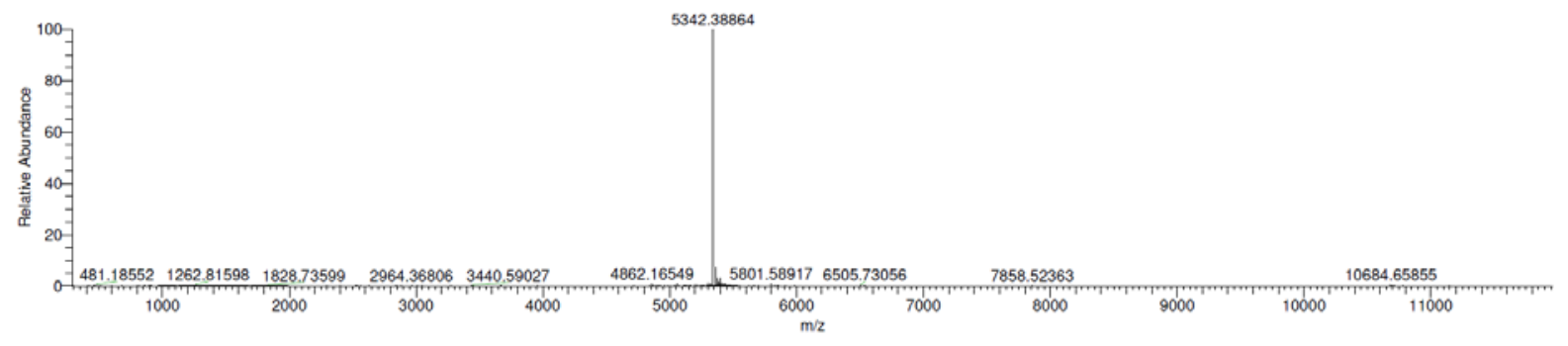

Figure S40. Upper panel: RP-HPLC analysis of analog 39 ([des (tetrapeptide ${ }^{\mathrm{B} 27-\mathrm{B} 30}$ ), $\left.\mathrm{Glu}^{\mathrm{B} 26}\right]-$ insulin). Lower panel: HR-MS of analog 39, Mw (monoisotopic for $\mathrm{C}_{234} \mathrm{H}_{348} \mathrm{~N}_{60} \mathrm{O}_{72} \mathrm{~S}_{6}$ ) calculated 5342.3738, Mw found 5342.3886. 

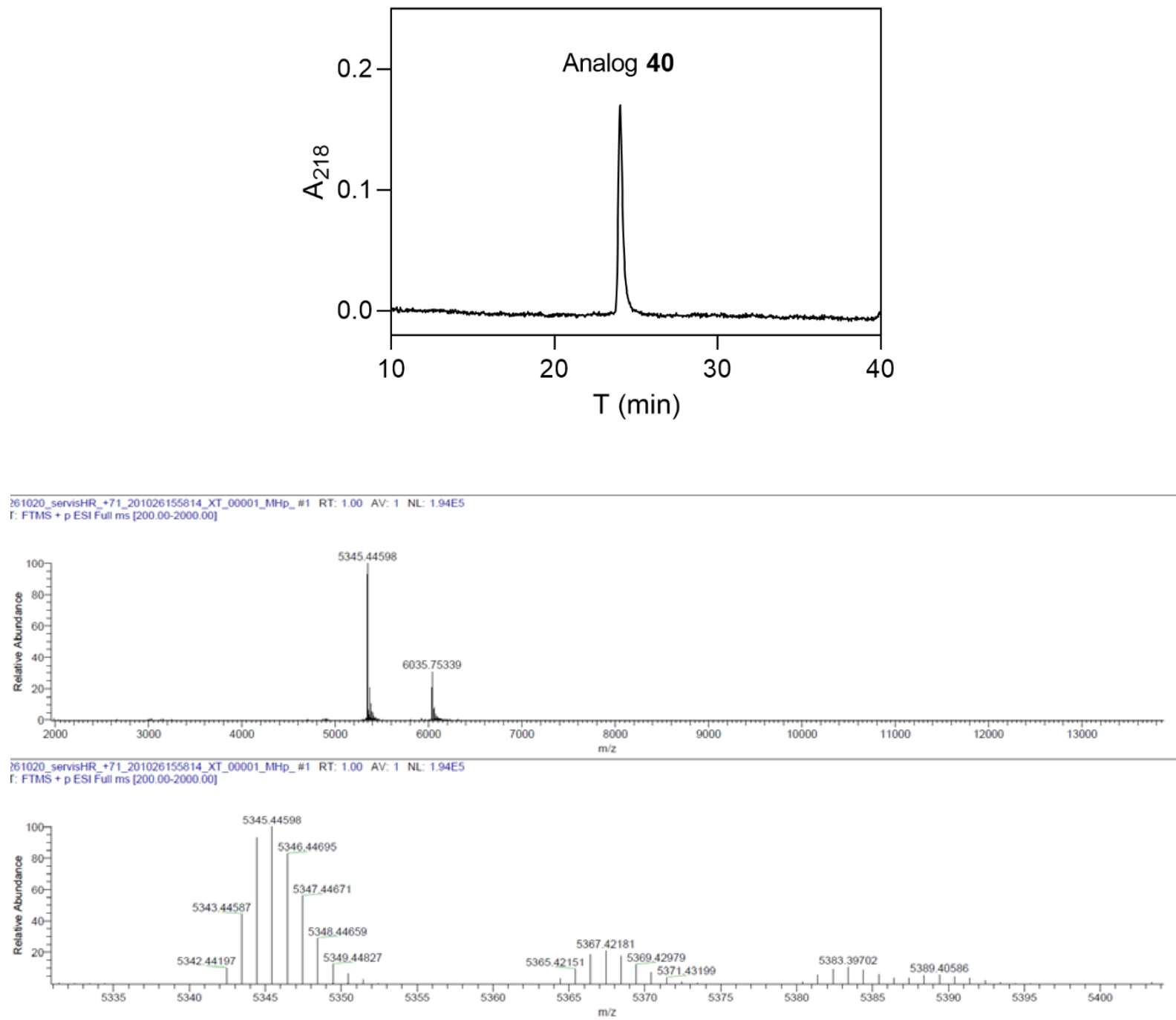

Figure S41. Upper panel: RP-HPLC analysis of analog 40 ([des(tetrapeptide ${ }^{\mathrm{B} 27-\mathrm{B} 30}$ ), Lys $\left.^{\mathrm{B} 26}\right]$ insulin). Lower panel: HR-MS of analog $40 \mathrm{Mw}$ (monoisotopic for $\mathrm{C}_{235} \mathrm{H}_{353} \mathrm{~N}_{61} \mathrm{O}_{70} \mathrm{~S}_{6}$ ) calculated 5341.4262, Mw found 5342.4420 $\left(\mathrm{MH}^{+}\right)$. 

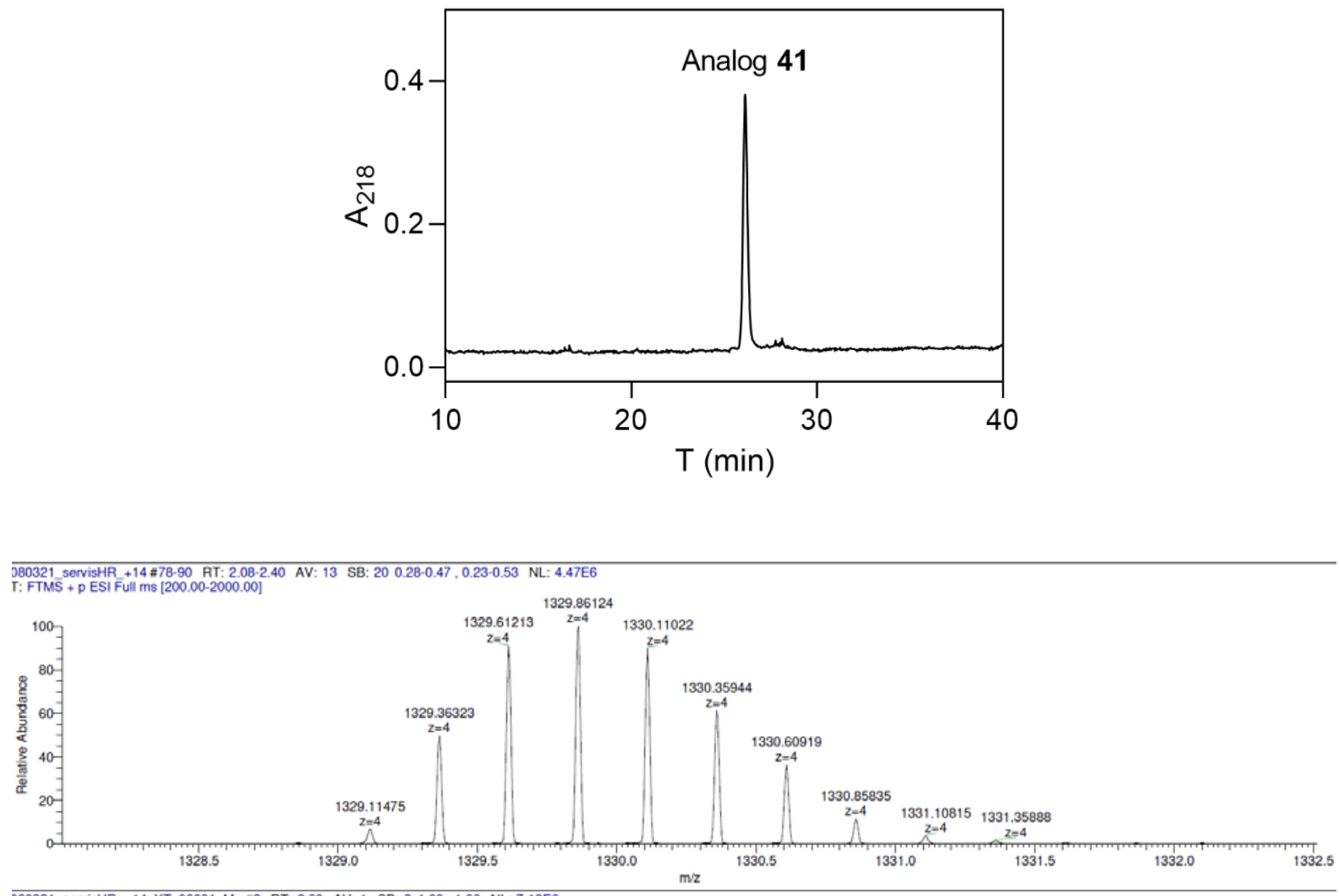

880321 servisHR_+14_XT_00001_M_\#2 RT: 2.00 AV: 1 SB: $21.00,1.00$ NL: $7.18 E 6$

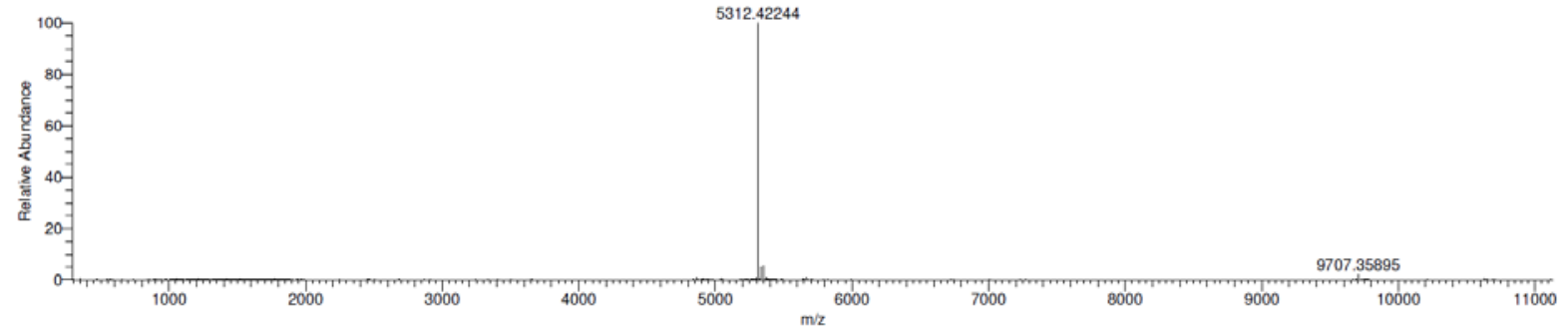

Figure S42. Upper panel: RP-HPLC analysis of analog 41 ([des(tetrapeptide ${ }^{\mathrm{B} 27-\mathrm{B} 30}$ ), $\left.\mathrm{Val}^{\mathrm{B} 26}\right]$ insulin). Lower panel: HR-MS of analog $41 \mathrm{Mw}$ (monoisotopic for $\mathrm{C}_{234} \mathrm{H}_{350} \mathrm{~N}_{60} \mathrm{O}_{70} \mathrm{~S}_{6}$ ) calculated 5312.3996, Mw found 5312.4224. 

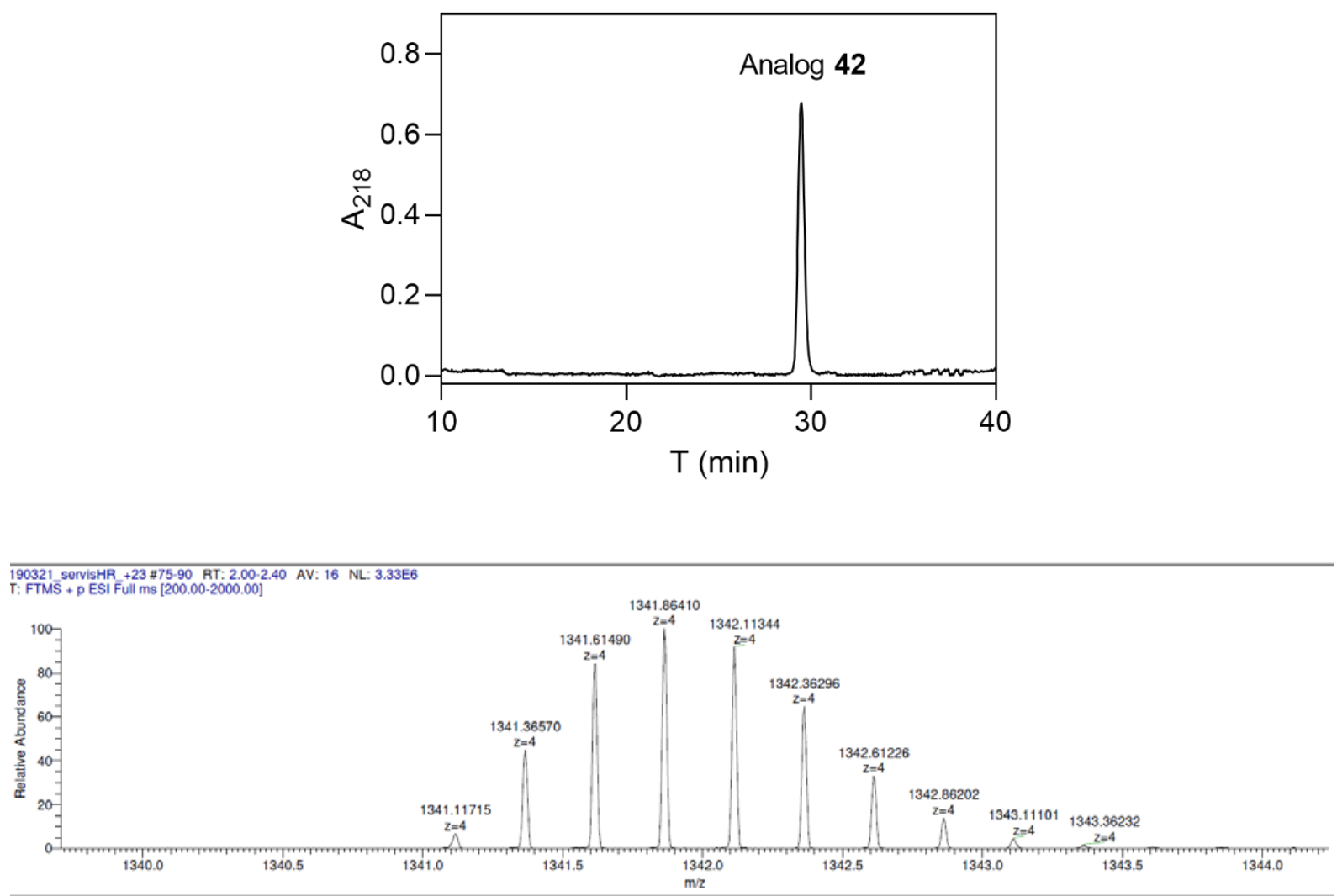

190321 sorvisHR_+23_XT_00001_M_\#2 RT: 2.00 AV: 1 NL: $4.86 E$ T: FTMS + P ESI Full ms [200.00-2000.00]

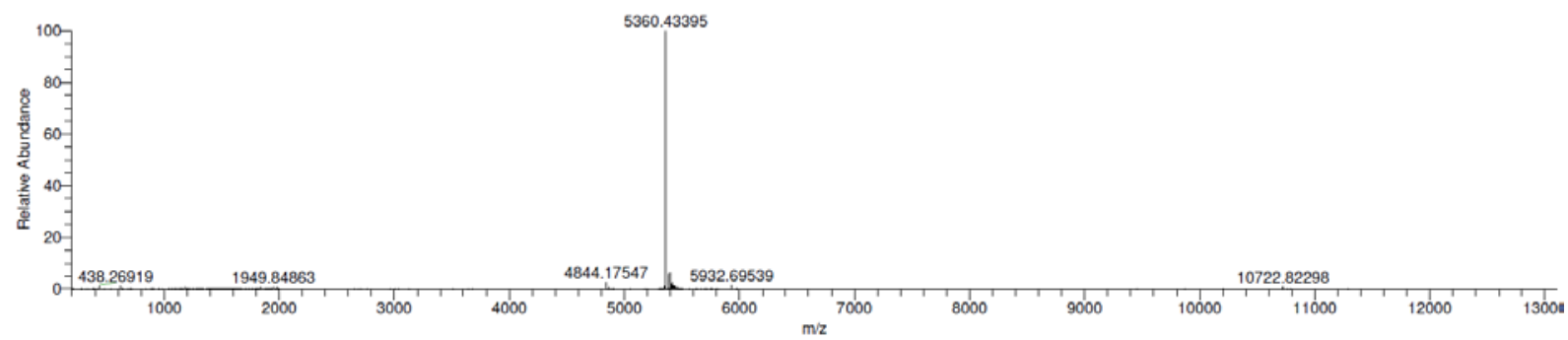

Figure S43. Upper panel: RP-HPLC analysis of analog 42 ([des (tetrapeptide ${ }^{\mathrm{B} 27-\mathrm{B} 30}$ ), $\left.\mathrm{Phe}^{\mathrm{B} 26}\right]$ insulin). Lower panel: HR-MS of analog $42 \mathrm{Mw}$ (monoisotopic for $\mathrm{C}_{238} \mathrm{H}_{350} \mathrm{~N}_{60} \mathrm{O}_{70} \mathrm{~S}_{6}$ ) calculated 5360.3996, Mw found 5360.4340. 

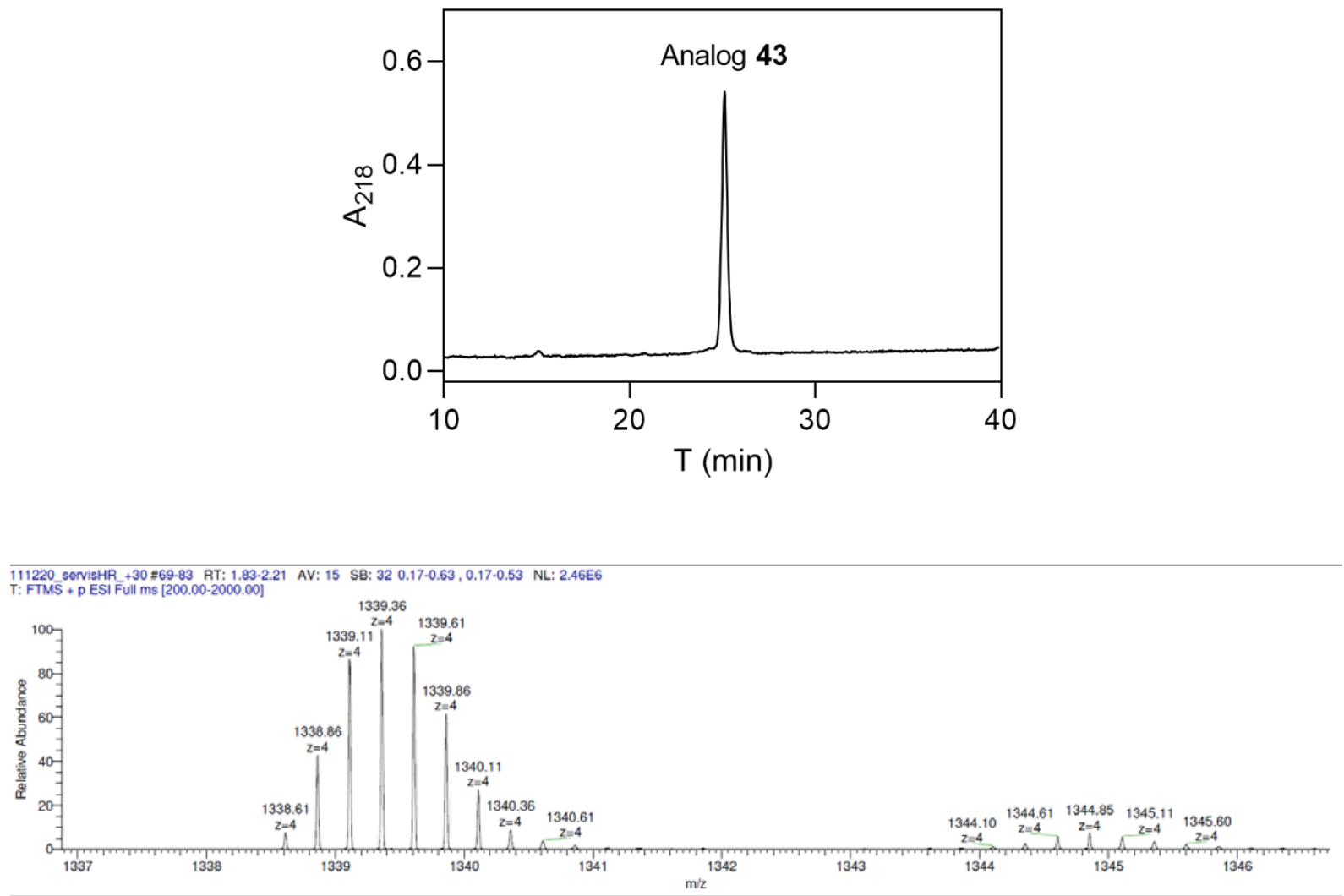

111220 servisHR_+30_XT_00001_M_ \#2 RT: 2.00 AV: 1 SB: $21.00,1.00$ NL: $4.15 E 6$
T: FTMS + + ESI Full ms $[200.00-2000.00]$

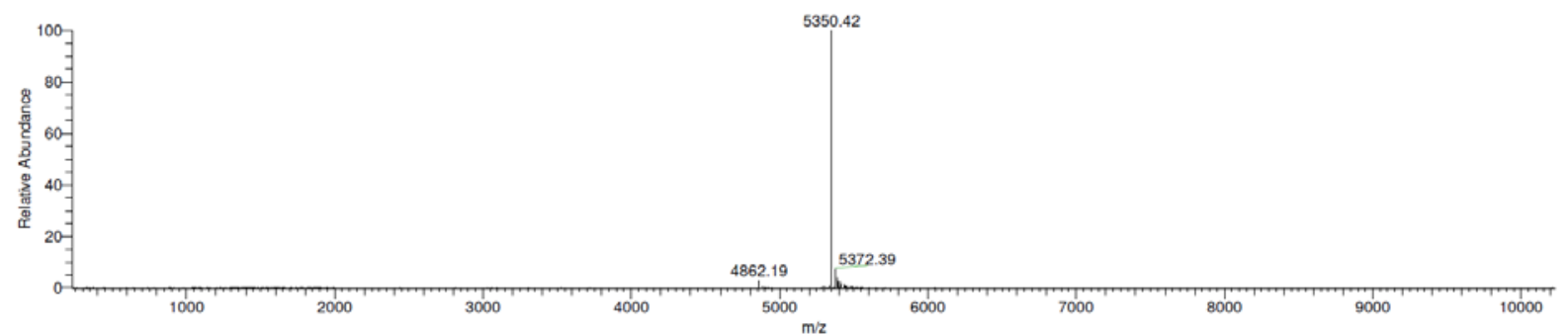

Figure S44. Upper panel: RP-HPLC analysis of analog 43 ([des(tetrapeptide ${ }^{\mathrm{B} 27-\mathrm{B} 30}$ ), His $\left.^{\mathrm{B} 26}\right]$ insulin). Lower panel: HR-MS of analog $43 \mathrm{Mw}$ (monoisotopic for $\mathrm{C}_{235} \mathrm{H}_{348} \mathrm{~N}_{62} \mathrm{O}_{70} \mathrm{~S}_{6}$ ) calculated 5350.3901, Mw found 5350.42. 

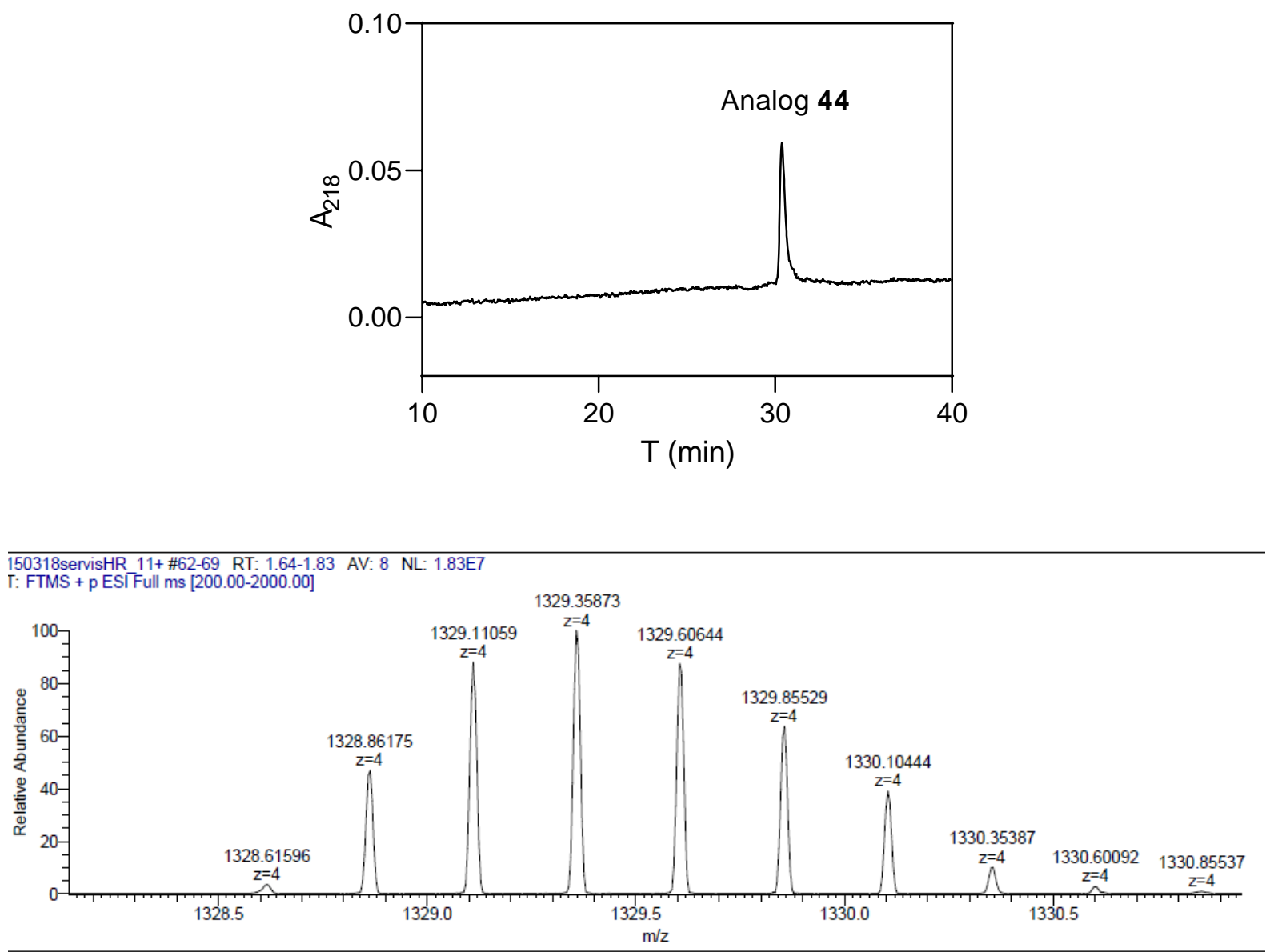

150318servisHR_11+_XT_00001_M \#2 RT: 2.00 AV: 1 NL: 3.16E7

T: FTMS + p ESĪ Full $\overline{\mathrm{ms}}[\overline{2} 00.00-\overline{2} 0 \overline{00} .00]$

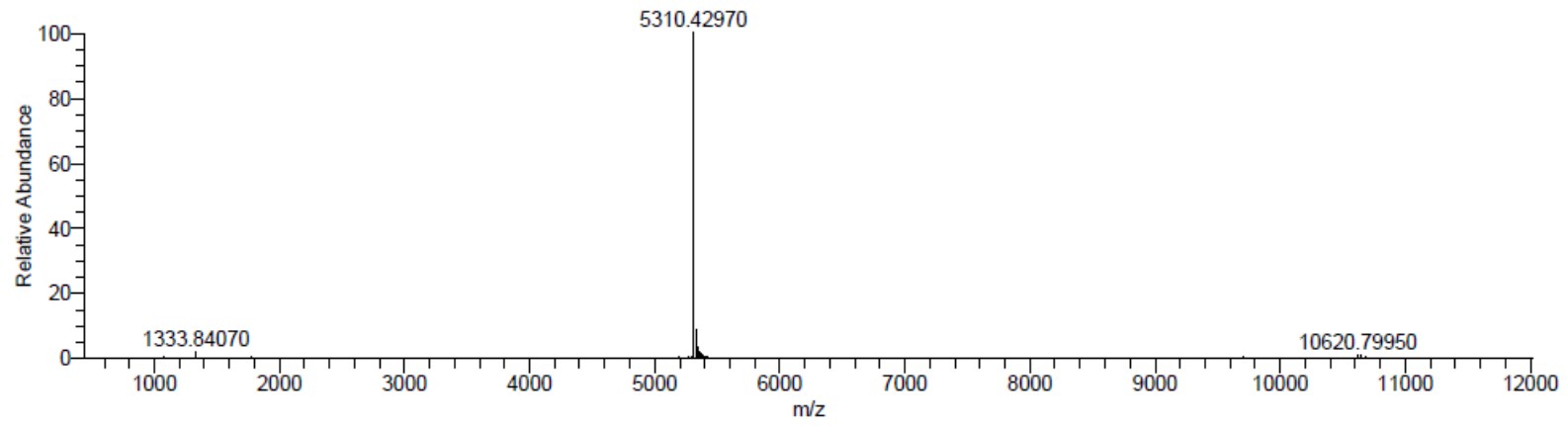

Figure S45. Upper panel: RP-HPLC analysis of analog 44 ([des(tetrapeptide ${ }^{\mathrm{B} 27-\mathrm{B} 30}$ ), Pro $\left.^{\mathrm{B} 26}\right]$ insulin). Lower panel: HR-MS of analog $44 \mathrm{Mw}$ (monoisotopic for $\mathrm{C}_{234} \mathrm{H}_{348} \mathrm{~N}_{60} \mathrm{O}_{70} \mathrm{~S}_{6}$ ) calculated 5310.3840, Mw found 5310.4297. 

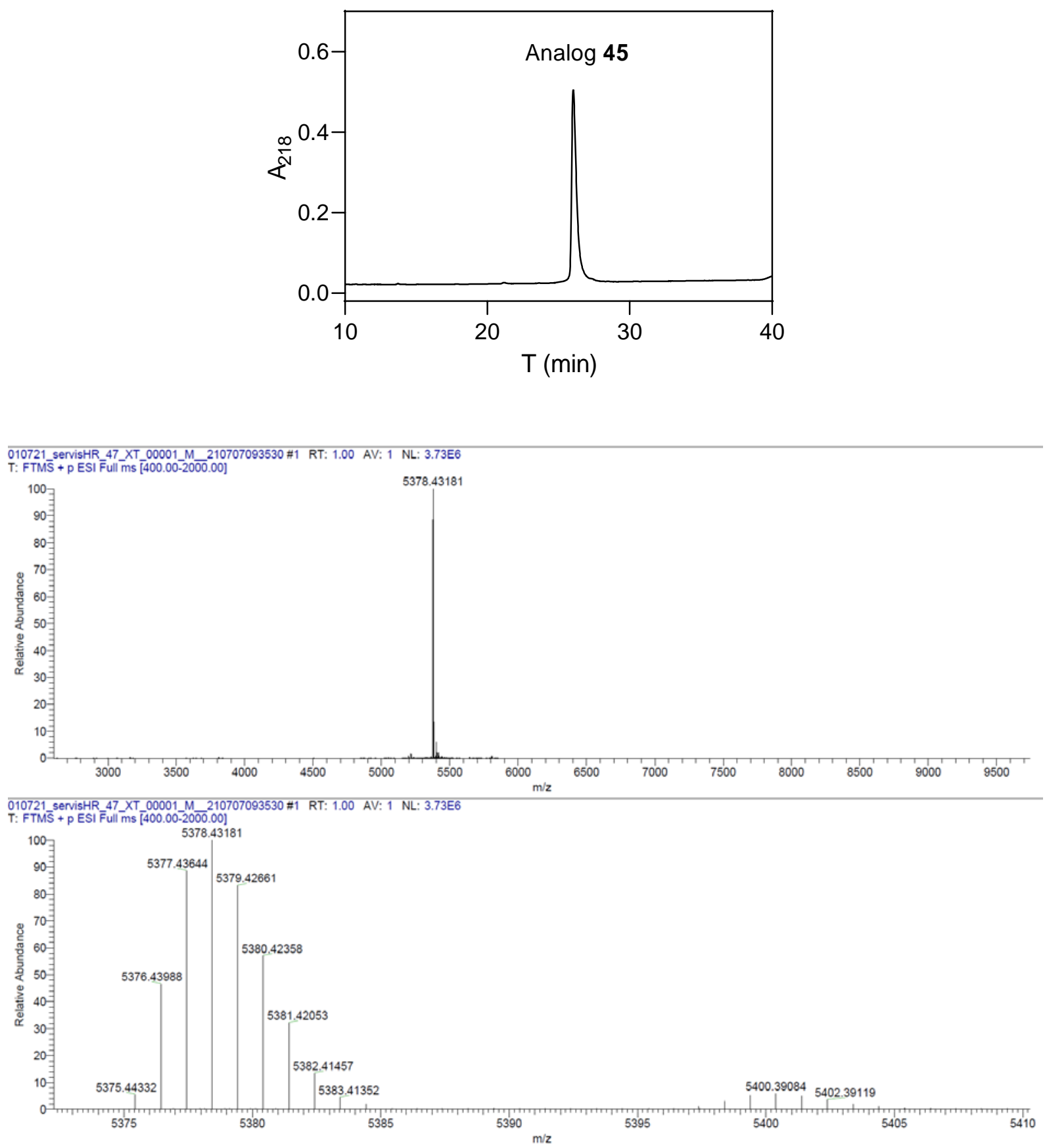

Figure S46. Upper panel: RP-HPLC analysis of analog 45 ([des(tetrapeptide ${ }^{\mathrm{B} 27-\mathrm{B} 30}$ ), amide $\left.^{\mathrm{B} 26}\right]$ insulin). Lower panel: HR-MS of analog $45 \mathrm{Mw}$ (monoisotopic for $\mathrm{C}_{238} \mathrm{H}_{351} \mathrm{~N}_{61} \mathrm{O}_{70} \mathrm{~S}_{6}$ ) calculated 5375.4105, Mw found 5375.4433. 


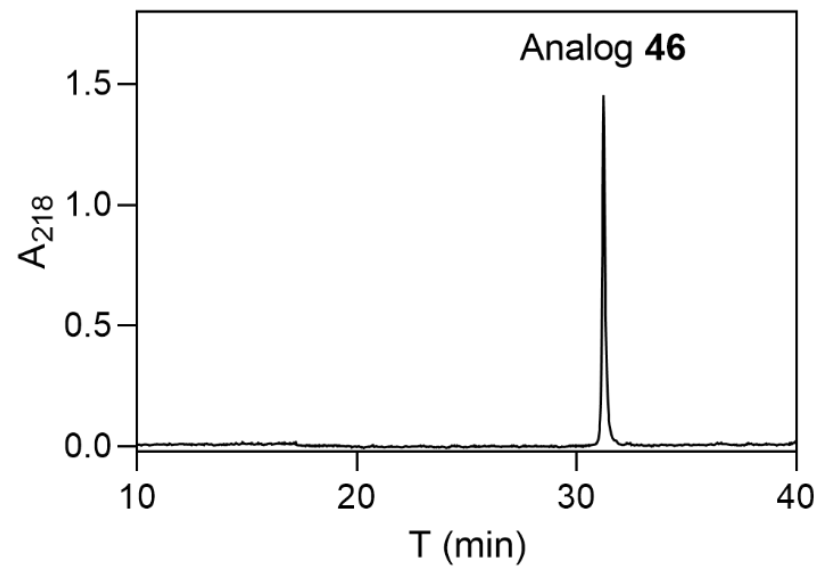

180621_servisHR_46_XT_00001_M_\#1 RT: 1.00 AV: 1 NL: $1.02 E 6$

T: FTMS + p ESI Full ms [200.00-2000.00]

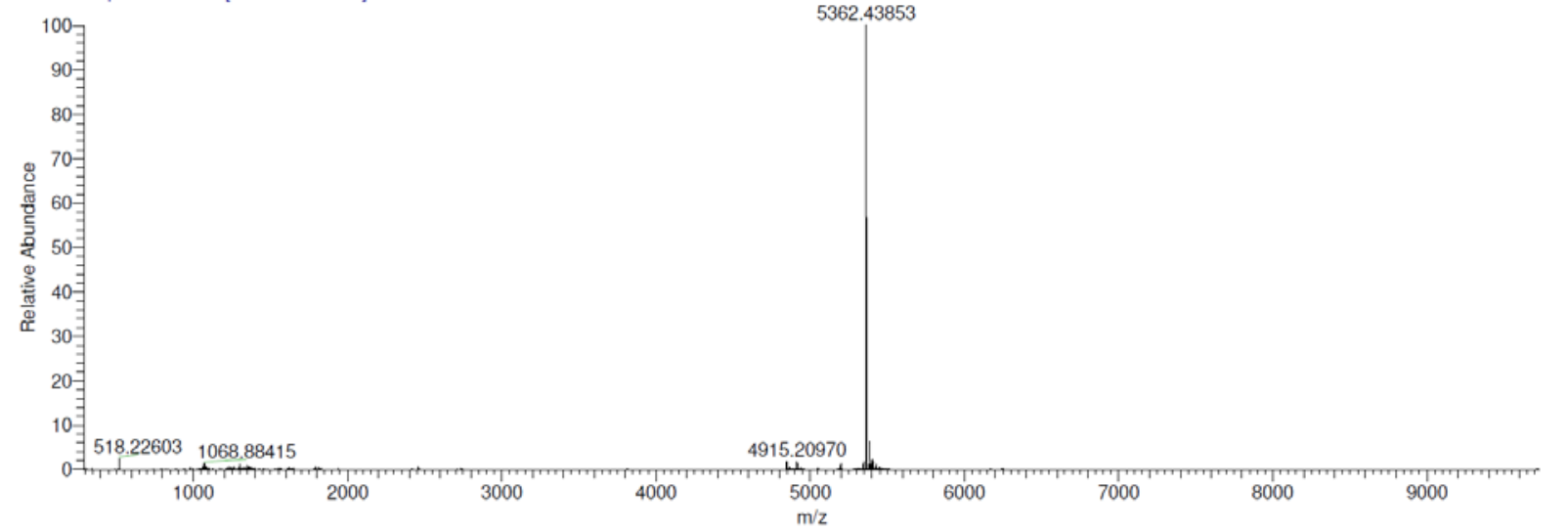

180621_servisHR_46_XT_00001_M_\#1 RT: 1.00 AV: 1 NL: $1.02 E 6$

T: FTM $\bar{S}+p$ ESI Full ms $\overline{[200.00-20 \overline{00.00]}}$

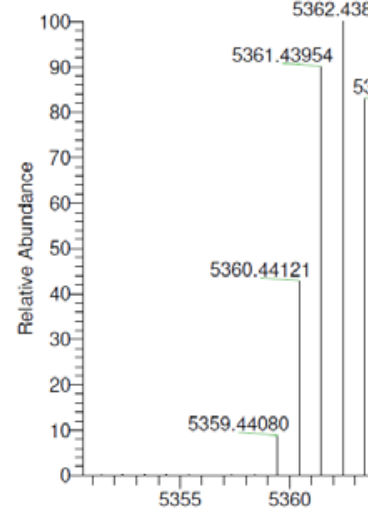

5363.43746

5364.43464

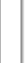

5365.43502

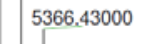

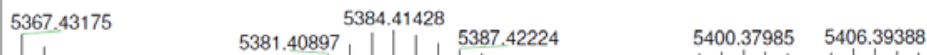

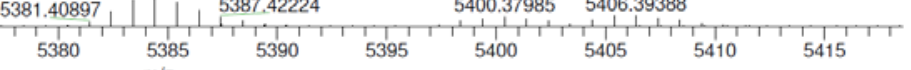

Figure S47. Upper panel: RP-HPLC analysis of analog 46 ([des(tetrapeptide ${ }^{\mathrm{B} 27-\mathrm{B} 30}$ ), $\mathrm{Phe}^{\mathrm{B} 26}$, amide ${ }^{\mathrm{B} 26}$ ]-insulin). Lower panel: HR-MS of analog $46 \mathrm{Mw}$ (monoisotopic for $\mathrm{C}_{238} \mathrm{H}_{351} \mathrm{~N}_{61} \mathrm{O}_{69} \mathrm{~S}_{6}$ ) calculated 5359.4156, Mw found 5359.4408. 

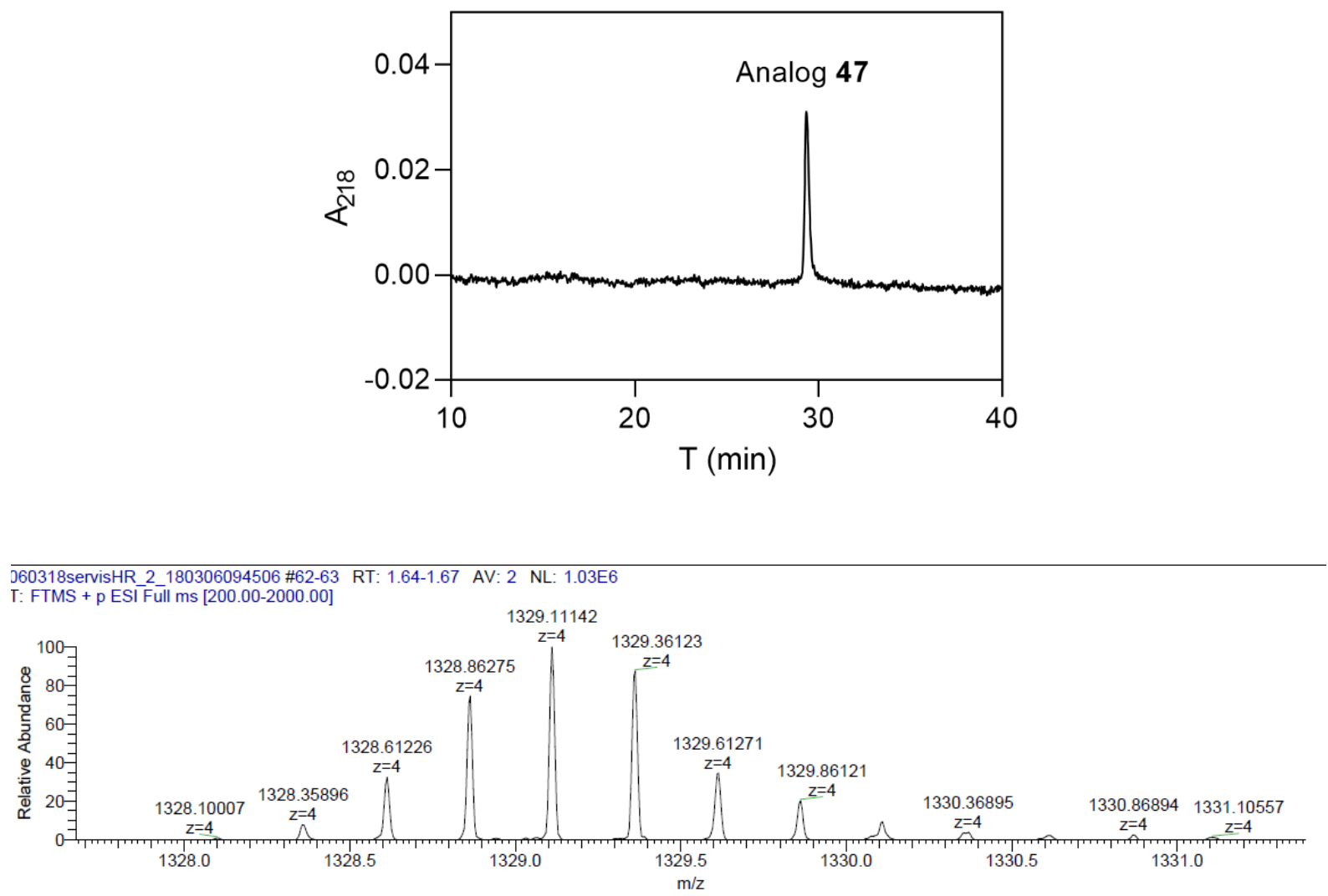

J60318servisHR_2_180306094506_XT_00001_M_\#2 RT: 2.00 AV: 1 NL: 1.36E6

T: FTMS + p ESİ Füll ms [200.00-2000.00]

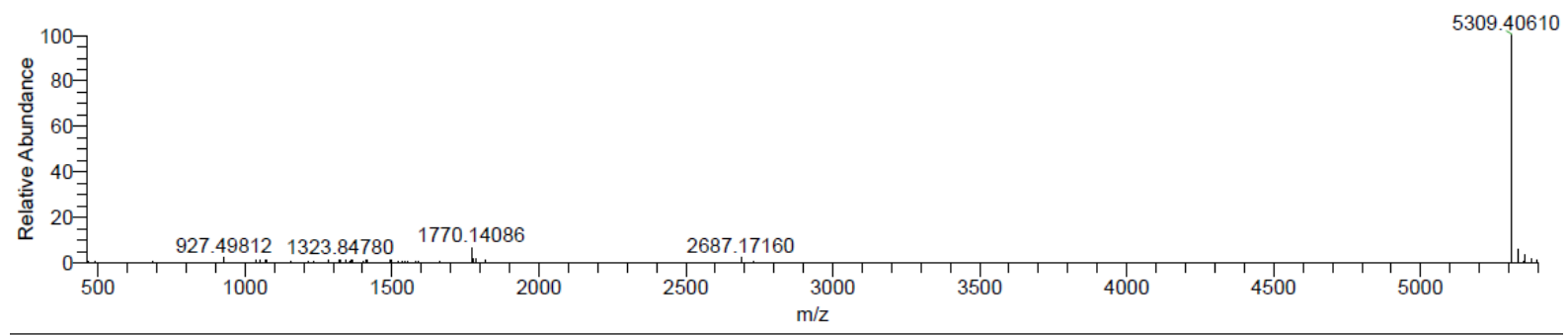

J60318servisHR_2_180306094506_XT_00001_M_\#2 RT: 2.00 AV: 1 NL: $1.36 E 6$

T: FTMS + p ESİ Füll ms [200.00-2000.00]

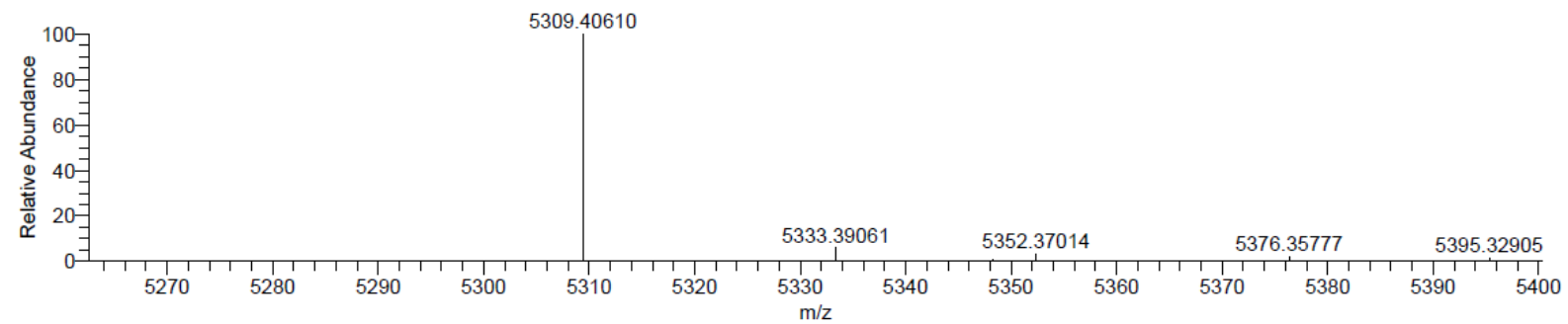

Figure S48. Upper panel: RP-HPLC analysis of analog 47 ([des (tetrapeptide ${ }^{\mathrm{B} 27-\mathrm{B} 30}$ ), $\mathrm{Pro}^{\mathrm{B} 26}$, amide ${ }^{\mathrm{B} 26}$ ]-insulin). Lower panel: $\mathrm{HR}-\mathrm{MS}$ of analog $47 \mathrm{Mw}$ (monoisotopic for $\mathrm{C}_{234} \mathrm{H}_{349} \mathrm{~N}_{61} \mathrm{O}_{69} \mathrm{~S}_{6}$ ) calculated 5309.4000, Mw found 5309.4061. 

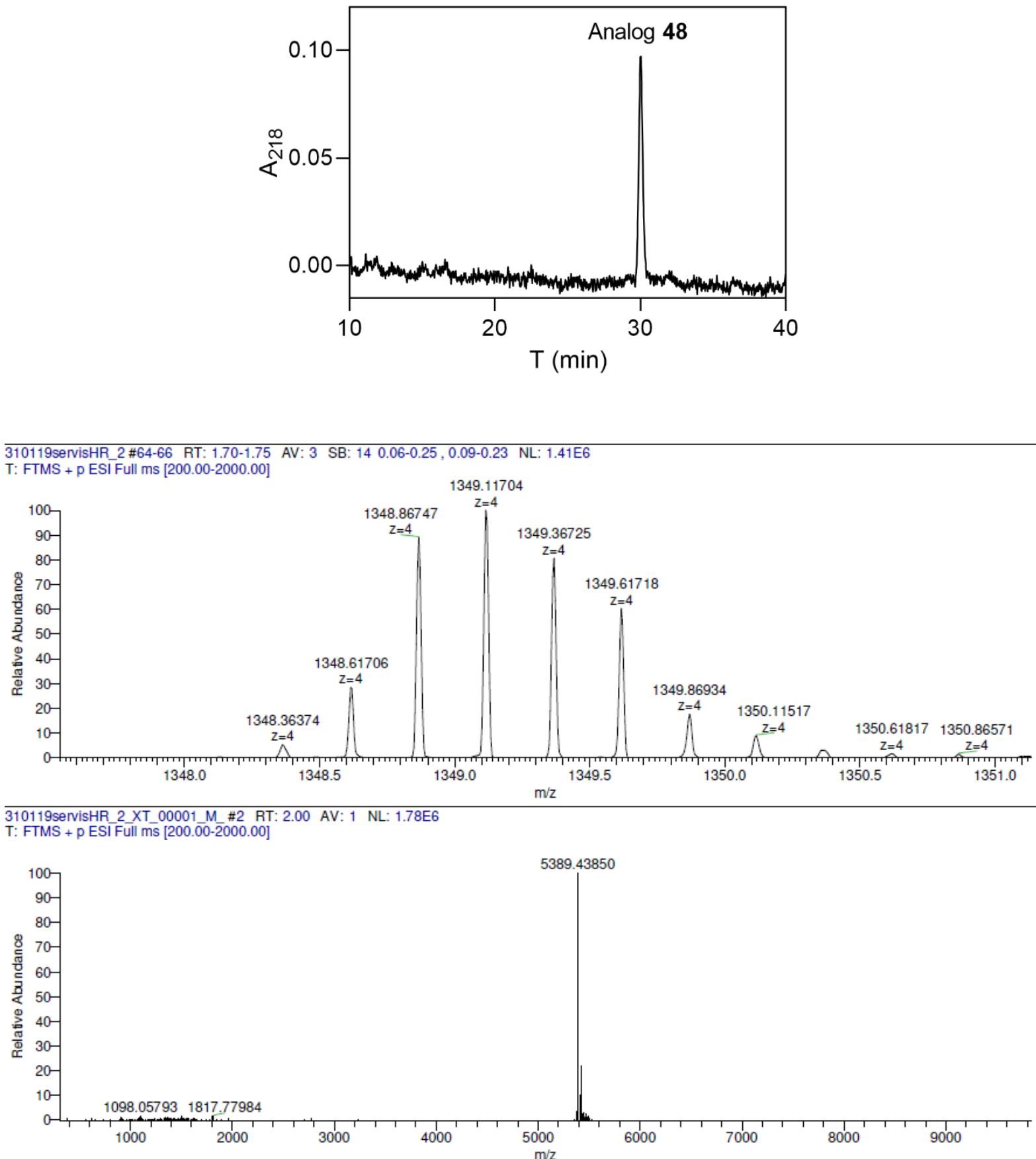

Figure S49. Upper panel: RP-HPLC analysis of analog 48 ([des(tetrapeptide ${ }^{\mathrm{B} 27-\mathrm{B} 30}$ ), $\mathrm{pTyr}^{\mathrm{B} 26}$, amide ${ }^{\mathrm{B} 26}$ ]-insulin). Lower panel: HR-MS of analog $48 \mathrm{Mw}$ (monoisotopic for $\mathrm{C}_{239} \mathrm{H}_{353} \mathrm{~N}_{61} \mathrm{O}_{70} \mathrm{~S}_{6}$ ) calculated 5389.4262, Mw found 5389.4385. 


\section{Receptor binding affinities of the analogs for IR-A and IR-B}

Table S2. Receptor-binding affinities of human insulin, analogs for human IR-A in membranes of IM-9 lymphocytes. The $K_{\mathrm{d}}$ values of human insulin and analogs were determined in three independent series of measurements indexed with a-c. The individual $K_{\mathrm{d}}$ values of analogs in the column are relative to a corresponding $K_{d}$ value of native insulin (e.g. a to a, etc.). Relative binding affinity is defined as ( $K_{d}$ of the native hormone / $K_{d}$ of analog) 100 (\%). Relative binding affinity S.D. values of analogs were calculated as S.D. $=K_{d}$ of the native hormone $/ K_{d}$ of analog x $\left.100 \times \sqrt{ }\left[\text { (S.D. native/ } K_{d} \text { native }\right)^{2}+\left(\text { S.D. analog } / K_{d} \text { analog) }\right)^{2}\right]$.

\begin{tabular}{llcc}
\hline Analog & $\begin{array}{c}K_{\mathrm{d}} \pm \text { S.D. } \\
\text { for IR-A } \\
{[\mathrm{nM}](\mathrm{n})}\end{array}$ & $\begin{array}{c}\text { Relative } \\
\text { binding affinity } \\
\text { (in \%) for IR-A } \\
\pm \text { S.D. }\end{array}$ \\
\hline & human insulin & $0.27 \pm 0.06(4)^{\mathrm{a}}$ & $100 \pm 22$ \\
& & $0.33 \pm 0.04(5)^{\mathrm{b}}$ & $100 \pm 12$ \\
& & $0.12 \pm 0.04(3)^{\mathrm{C}}$ & $100 \pm 33$ \\
$\mathbf{1}$ & {$\left[\mathrm{Asp}^{\mathrm{B} 27}, \mathrm{Thr}^{\mathrm{B} 28}, \mathrm{Ser}^{\mathrm{B} 29}, \mathrm{Ser}^{\mathrm{B} 30}\right]$-insulin } & $0.57 \pm 0.21(3)^{\mathrm{a}}$ & $47 \pm 20$ \\
$\mathbf{2}$ & {$\left[\mathrm{Glu}^{\mathrm{B} 27}, \mathrm{Thr}^{\mathrm{B} 28}, \mathrm{Ser}^{\mathrm{B} 29}, \mathrm{Ser}^{\mathrm{B} 30}\right]$-insulin } & $0.63 \pm 0.10(3)^{\mathrm{a}}$ & $43 \pm 12$ \\
$\mathbf{4}$ & {$\left[\mathrm{Glu}^{\mathrm{B} 28}, \mathrm{Thr}^{\mathrm{B} 29}, \mathrm{Ser}^{\mathrm{B} 30}, \mathrm{Ser}^{\mathrm{B} 31}\right]$-insulin } & $0.58 \pm 0.05(3)^{\mathrm{b}}$ & $57 \pm 8$ \\
$\mathbf{5}$ & {$\left[\mathrm{Asp}^{\mathrm{B} 31}, \mathrm{Thr}^{\mathrm{B} 32}, \mathrm{Ser}^{\mathrm{B} 33}, \mathrm{Ser}^{\mathrm{B} 34}\right]$-insulin } & $0.37 \pm 0.13(5)^{\mathrm{C}}$ & $32 \pm 15$ \\
\hline
\end{tabular}

Table S3. Receptor-binding affinities of human insulin, analogs for human IR-B in membranes of transfected mouse fibroblasts. The $K_{\mathrm{d}}$ values of human insulin and analogs were determined in three independent series of measurements indexed with a-c. The individual $K_{\mathrm{d}}$ values of analogs in the column are relative to a corresponding $K_{\mathrm{d}}$ value of native insulin (e.g. a to a, etc.). Relative binding affinity S.D. values of analogs were calculated as S.D. $=K_{\mathrm{d}}$ of the native hormone $/ K_{\mathrm{d}}$ of analog $\mathrm{x} 100 \mathrm{x} \sqrt{ }\left[\left(\text { S.D. native } / K_{\mathrm{d}} \text { native }\right)^{2}+\left(\text { S.D. analog } / K_{\mathrm{d}} \text { analog }\right)^{2}\right]$.

\begin{tabular}{llcr}
\hline Analog & $\begin{array}{c}K_{\mathrm{d}} \pm \text { S.D. } \\
\text { for IR-B } \\
{[\mathrm{nM}](\mathrm{n})}\end{array}$ & $\begin{array}{c}\text { Relative } \\
\text { binding affinity } \\
\text { (in \%) for IR-B } \\
\pm \text { S.D. }\end{array}$ \\
\hline & human insulin & $0.26 \pm 0.07(3)^{\mathrm{a}}$ & $100 \pm 27$ \\
& & $0.58 \pm 0.07(3)^{\mathrm{b}}$ & $100 \pm 12$ \\
& & $0.31 \pm 0.08(4)^{\mathrm{C}}$ & $100 \pm 26$ \\
$\mathbf{1}$ & {$\left[\mathrm{Asp}^{\mathrm{B} 27}, \mathrm{Thr}^{\mathrm{B} 28}, \mathrm{Ser}^{\mathrm{B} 29}, \mathrm{Ser}^{\mathrm{B} 30}\right]$-insulin } & $0.52 \pm 0.14(3)^{\mathrm{a}}$ & $50 \pm 19$ \\
$\mathbf{2}$ & {$\left[\mathrm{Glu}^{\mathrm{B} 27}, \mathrm{Thr}^{\mathrm{B} 28}, \mathrm{Ser}^{\mathrm{B} 29}, \mathrm{Ser}^{\mathrm{B} 30}\right]$-insulin } & $0.60 \pm 0.17(3)^{\mathrm{a}}$ & $43 \pm 17$ \\
$\mathbf{3}$ & {$\left[\mathrm{Glu}^{\mathrm{B} 28}, \mathrm{Thr}^{\mathrm{B} 29}, \mathrm{Ser}^{\mathrm{B} 30}, \mathrm{Ser}^{\mathrm{B} 31}\right]$-insulin } & $0.58 \pm 0.09(3)^{\mathrm{b}}$ & $100 \pm 20$ \\
$\mathbf{5}$ & {$\left[\mathrm{Asp}^{\mathrm{B} 31}, \mathrm{Thr}^{\mathrm{B} 32}, \mathrm{Ser}^{\mathrm{B} 33}, \mathrm{Ser}^{\mathrm{B} 34}\right]-$-insulin } & $0.53 \pm 0.21(4)^{\mathrm{C}}$ & $58 \pm 27$ \\
& {$\left[\mathrm{Asn}^{\mathrm{B} 26}, \mathrm{Asp}^{\mathrm{B} 31}, \mathrm{Thr}^{\mathrm{B} 32}, \mathrm{Ser}^{\mathrm{B} 33}, \mathrm{Ser}^{\mathrm{B} 34}\right]-$ insulin } & $0.68 \pm 0.15(3)^{\mathrm{C}}$ & $46 \pm 16$ \\
\hline
\end{tabular}


Table S4. Receptor-binding affinities of human insulin, analogs for human IR-A in membranes of IM-9 lymphocytes. The $K_{d}$ values of human insulin and analogs were determined in two independent series of measurements indexed with a-b. The individual $K_{d}$ values of analogs in the column are relative to a corresponding $K_{d}$ value of native insulin (e.g. a to a, etc.). Relative binding affinity is defined as ( $K_{d}$ of the native hormone / $K_{d}$ of analog) x 100 (\%). Relative binding affinity S.D. values of analogs were calculated as S.D. $=K_{d}$ of the native hormone $/ K_{\mathrm{d}}$ of analog x $100 \times \sqrt{ }\left[\left(\text { S.D. native/ } K_{\mathrm{d}} \text { native }\right)^{2}+\left(\text { S.D. analog } / K_{\mathrm{d}} \text { analog }\right)^{2}\right]$.

\begin{tabular}{llcc}
\hline & Analog & $\begin{array}{c}K_{\mathrm{d}} \pm \text { S.D. } \\
\text { for IR-A } \\
{[\mathrm{nM}](\mathrm{n})}\end{array}$ & $\begin{array}{c}\text { Relative } \\
\text { binding affinity } \\
\text { (in \%) for IR-A }\end{array}$ \\
\hline & human insulin & $0.32 \pm 0.09(4)^{\mathrm{a}}$ & $100 \pm 28$ \\
& & $0.30 \pm 0.13(5)^{\mathrm{b}}$ & $100 \pm 43$ \\
$\mathbf{6}$ & {$\left[\right.$ amide $\left.^{\mathrm{B} 30}\right]$-insulin } & $0.21 \pm 0.02(5)^{\mathrm{a}}$ & $152 \pm 45$ \\
$\mathbf{7}$ & {$\left[\mathrm{Gly}^{\mathrm{B} 31}\right.$, amide $\left.^{\mathrm{B} 31}\right]$-insulin } & $0.35 \pm 0.07(3)^{\mathrm{b}}$ & $86 \pm 41$ \\
$\mathbf{8}$ & {$\left[\mathrm{Gly}^{\mathrm{B} 31}, \mathrm{Gly}^{\mathrm{B} 32}\right.$, amide $\left.^{\mathrm{B} 32}\right]$-insulin } & $0.32 \pm 0.16(4)^{\mathrm{b}}$ & $94 \pm 62$ \\
$\mathbf{9}$ & {$\left[\mathrm{Gly}^{\mathrm{B} 31}, \mathrm{Gly}^{\mathrm{B} 32}, \mathrm{Gly}^{\mathrm{B} 33}\right.$, amide $\left.^{\mathrm{B} 33}\right]$-insulin } & $0.41 \pm 0.09(3)^{\mathrm{b}}$ & $73 \pm 35$ \\
\hline
\end{tabular}

Table S5. Receptor-binding affinities of human insulin, analogs for human IR-B in membranes of transfected mouse fibroblasts. The $K_{\mathrm{d}}$ values of human insulin and analogs were determined in two independent series of measurements indexed with a-b. The individual $K_{d}$ values of analogs in the column are relative to a corresponding $K_{d}$ value of native insulin (e.g. a to a, etc.). Relative binding affinity S.D. values of analogs were calculated as S.D. $=K_{d}$ of the native hormone $/ K_{d}$ of analog $\mathrm{x} 100 \times \sqrt{ }\left[\left(\text { S.D. native/ } K_{d} \text { native }\right)^{2}+\left(\text { S.D. analog } / K_{d} \text { analog }\right)^{2}\right]$.

\begin{tabular}{|c|c|c|c|}
\hline & Analog & $\begin{array}{c}K_{\mathrm{d}} \pm \text { S.D. } \\
\text { for IR-B } \\
{[\mathrm{nM}](\mathrm{n})}\end{array}$ & $\begin{array}{c}\text { Relative } \\
\text { binding affinity } \\
\text { (in \%) for IR-B } \\
\pm \text { S.D. }\end{array}$ \\
\hline & \multirow[t]{2}{*}{ human insulin } & $0.35 \pm 0.06(3)^{\mathrm{a}}$ & $100 \pm 17$ \\
\hline & & $0.50 \pm 0.32(5)^{b}$ & $100 \pm 64$ \\
\hline 6 & {$\left[\right.$ amide $^{\mathrm{B} 30}$ ]-insulin } & $0.17 \pm 0.18(4)^{\mathrm{a}}$ & $206 \pm 221$ \\
\hline 7 & {$\left[\mathrm{Gly}^{\mathrm{B} 31}\right.$, amide $\left.^{\mathrm{B} 31}\right]$-insulin } & $0.19 \pm 0.07(3)^{b}$ & $263 \pm 194$ \\
\hline 8 & {$\left[\mathrm{Gly}^{\mathrm{B} 31}\right.$, Gly $^{\mathrm{B} 32}$, amide $\left.^{\mathrm{B} 32}\right]$-insulin } & $0.30 \pm 0.21(4)^{b}$ & $167 \pm 158$ \\
\hline 9 & {$\left[\mathrm{Gly}^{\mathrm{B} 31}, \mathrm{Gly}^{\mathrm{B} 32}\right.$, Gly ${ }^{\mathrm{B} 33}$, amide $\left.{ }^{\mathrm{B} 33}\right]$-insulin } & $0.73 \pm 0.32(4)^{b}$ & $67 \pm 52$ \\
\hline
\end{tabular}


Table S6. Receptor-binding affinities of human insulin, analogs for human IR-A in membranes of IM-9 lymphocytes. The $K_{d}$ values of human insulin and analogs were determined in five independent series of measurements indexed with a-e. The individual $K_{\mathrm{d}}$ values of analogs in the column are relative to a corresponding $K_{d}$ value of native insulin (e.g. a to a, etc.). Relative binding affinity is defined as ( $K_{d}$ of the native hormone / $K_{d}$ of analog) x 100 (\%). Relative binding affinity S.D. values of analogs were calculated as S.D. $=K_{d}$ of the native hormone $/ K_{\mathrm{d}}$ of analog x $100 \times \sqrt{ }\left[\left(\text { S.D. native/ } K_{\mathrm{d}} \text { native }\right)^{2}+\left(\text { S.D. analog } / K_{\mathrm{d}} \text { analog }\right)^{2}\right]$.

\begin{tabular}{|c|c|c|c|}
\hline & Analog & $\begin{array}{c}K_{\mathrm{d}} \pm \text { S.D. } \\
\text { for IR-A } \\
{[\mathrm{nM}](\mathrm{n})}\end{array}$ & $\begin{array}{c}\text { Relative } \\
\text { binding affinity } \\
\text { (in \%) for IR-A } \\
\quad \pm \text { S.D. }\end{array}$ \\
\hline & \multirow[t]{5}{*}{ human insulin } & $0.33 \pm 0.04(5)^{\mathrm{a}}$ & $100 \pm 12$ \\
\hline & & $0.31 \pm 0.03(4)^{b}$ & $100 \pm 10$ \\
\hline & & $0.43 \pm 0.09(4)^{\mathrm{c}}$ & $100 \pm 21$ \\
\hline & & $0.42 \pm 0.12(3)^{d}$ & $100 \pm 29$ \\
\hline & & $0.32 \pm 0.02(3)^{\mathrm{e}}$ & $100 \pm 6$ \\
\hline 10 & {$\left[\mathrm{Glu}^{\mathrm{B} 31}\right.$, amide $\left.^{\mathrm{B} 31}\right]$-insulin } & $0.70 \pm 0.07(3)^{\mathrm{a}}$ & $47 \pm 7$ \\
\hline 11 & {$\left[\text { Lys }^{\mathrm{B} 28}, \text { Pro }^{\mathrm{B} 29} \text {, Gly }^{\mathrm{B} 31}\right]_{\text {-amide }}^{\mathrm{B} 31}$-insulin } & $0.42 \pm 0.02(3)^{\mathrm{a}}$ & $79 \pm 10$ \\
\hline 12 & {$\left[\mathrm{Ala}^{\mathrm{B} 29}, \mathrm{Gly}^{\mathrm{B} 31}\right.$, amide $\left.{ }^{\mathrm{B} 31}\right]$-insulin } & $0.75 \pm 0.15(3)^{\mathrm{a}}$ & $44 \pm 10$ \\
\hline 13 & {$\left[\mathrm{Ala}^{\mathrm{B} 29}, \mathrm{Asp}^{\mathrm{B} 31}\right.$, amide $\left.^{\mathrm{B} 31}\right]$-insulin } & $0.47 \pm 0.12(3)^{b}$ & $66 \pm 18$ \\
\hline 14 & {$\left[\mathrm{Ala}^{\mathrm{B} 29}, \mathrm{Glu}^{\mathrm{B} 3}\right.$, amide $\left.{ }^{\mathrm{B} 31}\right]$-insulin } & $0.97 \pm 0.55(4)^{\mathrm{c}}$ & $44 \pm 27$ \\
\hline 15 & {$\left[\mathrm{Ala}^{\mathrm{B} 29}, \mathrm{Glu}^{\mathrm{B} 31}\right]$-insulin } & $0.82 \pm 0.18(3)^{\mathrm{d}}$ & $51 \pm 18$ \\
\hline 16 & {$\left[\right.$ Lys $^{\mathrm{B} 28}$, Pro $\left.^{\mathrm{B} 29}, \mathrm{Glu}^{\mathrm{B} 31}\right]$-insulin } & $0.58 \pm 0.16(3)^{d}$ & $72 \pm 29$ \\
\hline 17 & {$\left[\mathrm{Glu}^{\mathrm{B} 31}\right]$-insulin } & $0.49 \pm 0.12(3)^{d}$ & $86 \pm 32$ \\
\hline 18 & {$\left[\mathrm{Ala}^{\mathrm{B} 29}, \mathrm{Gln}^{\mathrm{B} 31}\right.$, amide $\left.^{\mathrm{B} 31}\right]$-insulin } & $0.67 \pm 0.22(3)^{\mathrm{c}}$ & $64 \pm 25$ \\
\hline 19 & {$\left[\mathrm{Ala}^{\mathrm{B} 29}, \mathrm{His}^{\mathrm{B} 31}\right.$, amide $\left.^{\mathrm{B} 31}\right]$-insulin } & $0.36 \pm 0.11(3)^{\mathrm{c}}$ & $119 \pm 44$ \\
\hline 20 & {$\left[\mathrm{Ala}^{\mathrm{B} 29}, \mathrm{Ala}^{\mathrm{B} 31}\right.$, amide $\left.{ }^{\mathrm{B} 31}\right]$-insulin } & $0.57 \pm 0.15(3)^{\mathrm{c}}$ & $75 \pm 25$ \\
\hline 21 & {$\left[\mathrm{Ala}^{\mathrm{B} 29}, \mathrm{Thr}^{\mathrm{B} 31}\right.$, amide $^{\mathrm{B} 31}$ ]-insulin } & $0.54 \pm 0.11(3)^{\mathrm{c}}$ & $80 \pm 23$ \\
\hline 22 & {$\left[\mathrm{Ala}^{\mathrm{B} 29}, \mathrm{Tyr}^{\mathrm{B} 31}\right.$, amide $\left.^{\mathrm{B} 31}\right]$-insulin } & $0.68 \pm 0.09(3)^{\mathrm{c}}$ & $63 \pm 16$ \\
\hline 23 & {$\left[\mathrm{Ala}^{\mathrm{B} 29}, \mathrm{Met}^{\mathrm{B} 31}\right.$, amide $\left.^{\mathrm{B} 31}\right]$-insulin } & $0.41 \pm 0.05(3)^{\mathrm{c}}$ & $105 \pm 25$ \\
\hline 24 & {$\left[\mathrm{Ala}^{\mathrm{B} 29}, \mathrm{Ile}^{\mathrm{B} 31}\right.$, amide $\left.{ }^{\mathrm{B} 31}\right]$-insulin } & $0.91 \pm 0.22(3)^{\mathrm{c}}$ & $47 \pm 15$ \\
\hline 25 & {$\left[\mathrm{Ala}^{\mathrm{B} 29}, \operatorname{Trp}^{\mathrm{B} 31}\right.$, amide $\left.^{\mathrm{B} 31}\right]$-insulin } & $0.22 \pm 0.03(3)^{\mathrm{e}}$ & $145 \pm 22$ \\
\hline 26 & {$\left[\mathrm{Ala}^{\mathrm{B} 29}\right.$, Pro $^{\mathrm{B} 31}$, amide $\left.{ }^{\mathrm{B} 31}\right]$-insulin } & $0.54 \pm 0.06(3)^{\mathrm{e}}$ & $59 \pm 8$ \\
\hline
\end{tabular}


Table S7. Receptor-binding affinities of human insulin, analogs for human IR-B in membranes of transfected mouse fibroblasts. The $K_{\mathrm{d}}$ values of human insulin and analogs were determined in five independent series of measurements indexed with a-e. The individual $K_{d}$ values of analogs in the column are relative to a corresponding $K_{d}$ value of native insulin (e.g. a to a, etc.). Relative binding affinity S.D. values of analogs were calculated as S.D. $=K_{\mathrm{d}}$ of the native hormone $/ K_{\mathrm{d}}$ of analog x $100 \mathrm{x} \sqrt{ }\left[\left(\text { S.D. native/ } K_{\mathrm{d}} \text { native }\right)^{2}+\left(\right.\right.$ S.D. analog/K $K_{\mathrm{d}}$ analog) $\left.{ }^{2}\right]$.

\begin{tabular}{|c|c|c|c|}
\hline & Analog & $\begin{array}{c}K_{\mathrm{d}} \pm \text { S.D. } \\
\text { for IR-B } \\
{[\mathrm{nM}](\mathrm{n})}\end{array}$ & $\begin{array}{c}\text { Relative } \\
\text { binding affinity } \\
\text { (in \%) for IR-B } \\
\pm \text { S.D. }\end{array}$ \\
\hline & \multirow[t]{5}{*}{ human insulin } & $0.58 \pm 0.07(3)^{\mathrm{a}}$ & $100 \pm 12$ \\
\hline & & $0.32 \pm 0.15(3)^{b}$ & $100 \pm 47$ \\
\hline & & $0.49 \pm 0.18(3)^{\mathrm{c}}$ & $100 \pm 37$ \\
\hline & & $0.38 \pm 0.16(3)^{d}$ & $100 \pm 42$ \\
\hline & & $0.35 \pm 0.02(3)^{\mathrm{e}}$ & $100 \pm 6$ \\
\hline 10 & {$\left[\mathrm{Glu}^{\mathrm{B} 31}\right.$, amide $\left.^{\mathrm{B} 31}\right]$-insulin } & $0.40 \pm 0.05(3)^{\mathrm{a}}$ & $145 \pm 25$ \\
\hline 11 & {$\left[\text { Lys }^{\mathrm{B} 28}, \text { Pro }^{\mathrm{B} 29} \text {, Gly }^{\mathrm{B} 31}\right]_{\text {-amide }}^{\mathrm{B} 31}$-insulin } & $0.48 \pm 0.05(3)^{\mathrm{a}}$ & $121 \pm 19$ \\
\hline 12 & {$\left[\mathrm{Ala}^{\mathrm{B} 29}, \mathrm{Gly}^{\mathrm{B} 31}\right.$, amide $\left.{ }^{\mathrm{B} 31}\right]$-insulin } & $0.38 \pm 0.22(3)^{\mathrm{a}}$ & $153 \pm 90$ \\
\hline 13 & {$\left[\mathrm{Ala}^{\mathrm{B} 29}, \mathrm{Asp}^{\mathrm{B} 31}\right.$, amide $\left.^{\mathrm{B} 31}\right]$-insulin } & $0.51 \pm 0.08(3)^{b}$ & $63 \pm 31$ \\
\hline 14 & {$\left[\mathrm{Ala}^{\mathrm{B} 29}, \mathrm{Glu}^{\mathrm{B} 3}\right.$, amide $\left.^{\mathrm{B} 31}\right]$-insulin } & $0.35 \pm 0.04(3)^{c}$ & $140 \pm 54$ \\
\hline 15 & {$\left[\mathrm{Ala}^{\mathrm{B} 29}, \mathrm{Glu}^{\mathrm{B} 31}\right]$-insulin } & $0.26 \pm 0.09(3)^{\mathrm{d}}$ & $146 \pm 80$ \\
\hline 16 & {$\left[\right.$ Lys $^{\mathrm{B} 28}$, Pro $\left.^{\mathrm{B} 29}, \mathrm{Glu}^{\mathrm{B} 31}\right]$-insulin } & $0.40 \pm 0.07(3)^{d}$ & $95 \pm 43$ \\
\hline 17 & {$\left[\mathrm{Glu}^{\mathrm{B} 31}\right]$-insulin } & $0.48 \pm 0.08(3)^{d}$ & $79 \pm 36$ \\
\hline 18 & {$\left[\mathrm{Ala}^{\mathrm{B} 29}, \mathrm{Gln}^{\mathrm{B} 31}\right.$, amide $\left.^{\mathrm{B} 31}\right]$-insulin } & $0.37 \pm 0.03(3)^{\mathrm{c}}$ & $132 \pm 50$ \\
\hline 19 & {$\left[\mathrm{Ala}^{\mathrm{B} 29}, \mathrm{His}^{\mathrm{B} 31}\right.$, amide $\left.^{\mathrm{B} 31}\right]$-insulin } & $0.34 \pm 0.10(3)^{\mathrm{c}}$ & $144 \pm 68$ \\
\hline 20 & {$\left[\mathrm{Ala}^{\mathrm{B} 29}, \mathrm{Ala}^{\mathrm{B} 31}\right.$, amide $\left.^{\mathrm{B} 31}\right]$-insulin } & $0.53 \pm 0.25(3)^{\mathrm{c}}$ & $92 \pm 55$ \\
\hline 21 & {$\left[\mathrm{Ala}^{\mathrm{B} 29}, \mathrm{Thr}^{\mathrm{B} 31}\right.$, amide $\left.{ }^{\mathrm{B} 31}\right]$-insulin } & $0.45 \pm 0.06(3)^{\mathrm{c}}$ & $109 \pm 43$ \\
\hline 22 & {$\left[\mathrm{Ala}^{\mathrm{B} 29}, \mathrm{Tyr}^{\mathrm{B} 31}\right.$, amide $\left.^{\mathrm{B} 31}\right]$-insulin } & $0.39 \pm 0.05(3)^{\mathrm{c}}$ & $126 \pm 49$ \\
\hline 23 & {$\left[\mathrm{Ala}^{\mathrm{B} 29}, \mathrm{Met}^{\mathrm{B} 31}\right.$, amide $\left.^{\mathrm{B} 31}\right]$-insulin } & $0.46 \pm 0.19(3)^{\mathrm{c}}$ & $107 \pm 59$ \\
\hline 24 & {$\left[\mathrm{Ala}^{\mathrm{B} 29}, \mathrm{Ile}^{\mathrm{B} 31}\right.$, amide $\left.^{\mathrm{B} 31}\right]$-insulin } & $0.63 \pm 0.05(3)^{c}$ & $78 \pm 29$ \\
\hline 25 & {$\left[\mathrm{Ala}^{\mathrm{B} 29}, \operatorname{Trp}^{\mathrm{B} 31}\right.$, amide $\left.^{\mathrm{B} 31}\right]$-insulin } & $0.44 \pm 0.18(3)^{\mathrm{e}}$ & $80 \pm 33$ \\
\hline 26 & {$\left[\mathrm{Ala}^{\mathrm{B} 29}\right.$, Pro $^{\mathrm{B} 31}$, amide $\left.^{\mathrm{B} 31}\right]$-insulin } & $0.65 \pm 0.03(3)^{\mathrm{e}}$ & $54 \pm 4$ \\
\hline
\end{tabular}


Table S8. Receptor-binding affinities of human insulin, analogs for human IR-A in membranes of IM-9 lymphocytes. The $K_{d}$ values of human insulin and analogs were determined in one independent series of measurements. The individual $K_{\mathrm{d}}$ values of analogs in the column are relative to a corresponding $K_{\mathrm{d}}$ value of native insulin. Relative binding affinity is defined as ( $K_{d}$ of the native hormone / $K_{d}$ of analog) x 100 (\%). Relative binding affinity S.D. values of analogs were calculated as S.D. $=K_{d}$ of the native hormone $/ K_{d}$ of analog x $100 \mathrm{x}$ $\left.\sqrt{ }\left[\text { (S.D. native/ } K_{\mathrm{d}} \text { native }\right)^{2}+\left(\text { S.D. analog } / K_{\mathrm{d}} \text { analog }\right)^{2}\right]$.

\begin{tabular}{|c|c|c|c|}
\hline & Analog & $\begin{array}{l}K_{\mathrm{d}} \pm \text { S.D. } \\
\text { for IR-A } \\
{[\mathrm{nM}](\mathrm{n})}\end{array}$ & $\begin{array}{c}\text { Relative } \\
\text { binding affinity } \\
\text { (in \%) for IR-A } \\
\pm \text { S.D. }\end{array}$ \\
\hline & human insulin & $0.31 \pm 0.03$ & $100 \pm 10$ \\
\hline 27 & {$\left[\mathrm{Ala}^{\mathrm{B} 29}, \mathrm{D}-\mathrm{Glu}^{\mathrm{B} 31}\right.$, amide $\left.^{\mathrm{B} 31}\right]$-insulin } & $0.39 \pm 0.10$ & $79 \pm 22$ \\
\hline 28 & {$\left[\mathrm{Ala}^{\mathrm{B} 29}, \mathrm{hGlu}^{\mathrm{B} 31}\right.$, amide $\left.^{\mathrm{B} 31}\right]$-insulin } & $0.44 \pm 0.04$ & $70 \pm 9$ \\
\hline 29 & {$\left[\mathrm{Ala}^{\mathrm{B} 29}, \mathrm{Gla}^{\mathrm{B} 31}\right.$, amide $\left.^{\mathrm{B} 31}\right]$-insulin } & $0.37 \pm 0.08$ & $84 \pm 20$ \\
\hline
\end{tabular}

Table S9. Receptor-binding affinities of human insulin, analogs for human IR-B in membranes of transfected mouse fibroblasts. The $K_{\mathrm{d}}$ values of human insulin and analogs were determined in one independent series of measurements. The individual $K_{\mathrm{d}}$ values of analogs in the column are relative to a corresponding $K_{\mathrm{d}}$ value of native insulin. Relative binding affinity S.D. values of analogs were calculated as S.D. $=K_{d}$ of the native hormone $/ K_{\mathrm{d}}$ of analog x $100 \times \sqrt{ }\left[\left(\text { S.D. native/ } K_{\mathrm{d}} \text { native }\right)^{2}+\left(\text { S.D. analog } / K_{\mathrm{d}} \text { analog }\right)^{2}\right]$.

\begin{tabular}{llcc}
\hline & Analog & $\begin{array}{c}K_{\mathrm{d}} \pm \mathrm{S} . \mathrm{D} . \\
\text { for IR-B } \\
{[\mathrm{nM}](\mathrm{n})}\end{array}$ & $\begin{array}{c}\text { Relative } \\
\text { binding affinity } \\
\text { (in \%) for IR-B } \\
\pm \text { S.D. }\end{array}$ \\
\hline $\mathbf{2 7}$ & human insulin & $0.32 \pm 0.15(3)$ & $100 \pm 47$ \\
$\mathbf{2 8}$ & {$\left[\mathrm{Ala}^{\mathrm{B} 29}, \mathrm{D}-\mathrm{Glu}^{\mathrm{B} 31}\right.$, amide $\left.^{\mathrm{B} 31}\right]$-insulin } & $0.94 \pm 0.21(3)$ & $34 \pm 18$ \\
$\mathbf{2 9}$ & {$\left[\mathrm{Ala}^{\mathrm{B} 29}, \mathrm{hGlu}^{\mathrm{B} 31}\right.$, amide $\left.^{\mathrm{B} 31}\right]$-insulin } & $0.72 \pm 0.39(3)$ & $44 \pm 32$ \\
\hline
\end{tabular}


Table S10. Receptor-binding affinities of human insulin, analogs for human IR-A in membranes of IM-9 lymphocytes. The $K_{\mathrm{d}}$ values of human insulin and analogs were determined in two independent series of measurements indexed with a-b. The individual $K_{d}$ values of analogs in the column are relative to a corresponding $K_{d}$ value of native insulin (e.g. a to a, etc.). Relative binding affinity is defined as ( $K_{d}$ of the native hormone / $K_{d}$ of analog) x 100 (\%). Relative binding affinity S.D. values of analogs were calculated as S.D. $=K_{d}$ of the native hormone $/ K_{\mathrm{d}}$ of analog x $100 \times \sqrt{ }\left[\left(\text { S.D. native/ } K_{\mathrm{d}} \text { native }\right)^{2}+\left(\text { S.D. analog } / K_{\mathrm{d}} \text { analog }\right)^{2}\right]$.

\begin{tabular}{|c|c|c|c|}
\hline & Analog & $\begin{array}{c}K_{\mathrm{d}} \pm \text { S.D. } \\
\text { for IR-A } \\
{[\mathrm{nM}](\mathrm{n})}\end{array}$ & $\begin{array}{c}\text { Relative } \\
\text { binding affinity } \\
\text { (in \%) for IR-A } \\
\quad \pm \text { S.D. }\end{array}$ \\
\hline & \multirow[t]{2}{*}{ human insulin } & $0.29 \pm 0.04(3)^{\mathrm{a}}$ & $100 \pm 14$ \\
\hline & & $0.33 \pm 0.04(3)^{b}$ & $100 \pm 12$ \\
\hline 30 & {$\left[\mathrm{Glu}^{\mathrm{A} 18}\right]$-insulin } & $0.80 \pm 0.05(3)^{a}$ & $36 \pm 5$ \\
\hline 31 & {$\left[\mathrm{Glu}^{\mathrm{A} 14}\right]$-insulin } & $0.49 \pm 0.06(3)^{\mathrm{a}}$ & $59 \pm 11$ \\
\hline 32 & {$\left[\right.$ Gly $\left.^{\mathrm{A}-1}\right]$-insulin } & $0.86 \pm 0.12(3)^{b}$ & $38 \pm 7$ \\
\hline 33 & {$\left[\right.$ Gly $^{\mathrm{A}-2}$, Gly $\left.^{\mathrm{A}-1}\right]$-insulin } & $0.85 \pm 0.13(3)^{b}$ & $39 \pm 8$ \\
\hline 34 & {$\left[\mathrm{Gly}^{\mathrm{A}-3}\right.$, Gly $^{\mathrm{A}-2}$, Gly $\left.{ }^{\mathrm{A}-1}\right]$-insulin } & $1.99 \pm 0.54(3)^{\mathrm{b}}$ & $17 \pm 5$ \\
\hline
\end{tabular}

Table S11. Receptor-binding affinities of human insulin, analogs for human IR-B in membranes of transfected mouse fibroblasts. The $K_{d}$ values of human insulin and analogs were determined in two independent series of measurements indexed with a-b. The individual $K_{\mathrm{d}}$ values of analogs in the column are relative to a corresponding $K_{\mathrm{d}}$ value of native insulin (e.g. a to a, etc.). Relative binding affinity S.D. values of analogs were calculated as S.D. $=K_{\mathrm{d}}$ of the native hormone $/ K_{\mathrm{d}}$ of analog x $100 \mathrm{x} \sqrt{ }\left[\left(\text { S.D. native/ } K_{\mathrm{d}} \text { native }\right)^{2}+\left(\right.\right.$ S.D. analog/ $K_{\mathrm{d}}$ analog) $\left.{ }^{2}\right]$.

\begin{tabular}{|c|c|c|c|}
\hline & Analog & $\begin{array}{l}K_{\mathrm{d}} \pm \text { S.D. } \\
\text { for IR-B } \\
{[\mathrm{nM}](\mathrm{n})}\end{array}$ & $\begin{array}{c}\text { Relative } \\
\text { binding affinity } \\
\text { (in \%) for IR-B } \\
\pm \text { S.D. }\end{array}$ \\
\hline & \multirow[t]{2}{*}{ human insulin } & $0.32 \pm 0.15(3)^{\mathrm{a}}$ & $100 \pm 47$ \\
\hline & & $0.58 \pm 0.07(3)^{b}$ & $100 \pm 12$ \\
\hline 30 & {$\left[\mathrm{Glu}^{\mathrm{A} 18}\right]$-insulin } & $3.49 \pm 0.29(3)^{a}$ & $9 \pm 4$ \\
\hline 31 & {$\left[\mathrm{Glu}^{\mathrm{A} 14}\right]$-insulin } & $1.30 \pm 0.39(3)^{\mathrm{a}}$ & $25 \pm 14$ \\
\hline 32 & {$\left[\mathrm{Gly}^{\mathrm{A}-1}\right]$-insulin } & $1.24 \pm 0.44(3)^{\mathrm{b}}$ & $47 \pm 18$ \\
\hline 33 & {$\left[\mathrm{Gly}^{\mathrm{A}-2}\right.$, Gly $\left.^{\mathrm{A}-1}\right]$-insulin } & $1.30 \pm 0.14(3)^{b}$ & $45 \pm 7$ \\
\hline 34 & {$\left[\mathrm{Gly}^{\mathrm{A}-3}\right.$, Gly $^{\mathrm{A}-2}$, Gly $\left.^{\mathrm{A}-1}\right]$-insulin } & $1.97 \pm 0.25(3)^{b}$ & $29 \pm 5$ \\
\hline
\end{tabular}


Table S12. Receptor-binding affinities of human insulin, analogs for human IR-A in membranes of IM-9 lymphocytes. The $K_{d}$ values of human insulin and analogs were determined in eight independent series of measurements indexed with a-h. The individual $K_{d}$ values of analogs in the column are relative to a corresponding $K_{d}$ value of native insulin (e.g. a to a, etc.). Relative binding affinity is defined as ( $K_{d}$ of the native hormone / $K_{d}$ of analog) x 100 (\%). Relative binding affinity S.D. values of analogs were calculated as S.D. $=K_{d}$ of the native hormone $/ K_{\mathrm{d}}$ of analog x $100 \times \sqrt{ }\left[\left(\text { S.D. native/ } K_{\mathrm{d}} \text { native }\right)^{2}+\left(\text { S.D. analog } / K_{\mathrm{d}} \text { analog }\right)^{2}\right]$.

\begin{tabular}{|c|c|c|c|}
\hline & Analog & $\begin{array}{l}K_{\mathrm{d}} \pm \text { S.D. } \\
\text { for IR-A } \\
{[\mathrm{nM}](\mathrm{n})}\end{array}$ & $\begin{array}{l}\text { Relative } \\
\text { binding affinity } \\
\text { (in \%) for IR-A }\end{array}$ \\
\hline & \multirow[t]{8}{*}{ human insulin } & $0.34 \pm 0.11(3)^{\mathrm{a}}$ & $100 \pm 32$ \\
\hline & & $0.42 \pm 0.12(3)^{b}$ & $100 \pm 29$ \\
\hline & & $0.32 \pm 0.02(3)^{\mathrm{c}}$ & $100 \pm 6$ \\
\hline & & $0.27 \pm 0.08(3)^{\mathrm{d}}$ & $100 \pm 30$ \\
\hline & & $0.28 \pm 0.03(3)^{\mathrm{e}}$ & $100 \pm 11$ \\
\hline & & $0.27 \pm 0.02(3)^{f}$ & $100 \pm 7$ \\
\hline & & $0.43 \pm 0.01(5)^{g}$ & $100 \pm 2$ \\
\hline & & $0.27 \pm 0.06(4)^{\mathrm{h}}$ & $100 \pm 22$ \\
\hline 35 & [des(tetrapeptide $\left.{ }^{\mathrm{B} 27-\mathrm{B} 30}\right)$ ]-insulin & $1.23 \pm 0.27(3)^{\mathrm{a}}$ & $28 \pm 11$ \\
\hline 36 & [des(tetrapeptide $\left.{ }^{\mathrm{B} 27-\mathrm{B} 30}\right)$, Gly ${ }^{\mathrm{B} 26}$ ]-insulin & $0.21 \pm 0.01(3)^{b}$ & $200 \pm 58$ \\
\hline 37 & [des(tetrapeptide $\left.{ }^{\mathrm{B} 27-\mathrm{B} 30}\right)$, Ser $\left.\left.{ }^{\mathrm{B} 26}\right)\right]$-insulin & $0.18 \pm 0.04(3)^{\mathrm{c}}$ & $178 \pm 41$ \\
\hline 38 & [des(tetrapeptide $\left.\left.{ }^{\mathrm{B} 27-\mathrm{B} 30}\right), \mathrm{Thr}^{\mathrm{B} 26}\right]$-insulin & $1.05 \pm 0.04(3)^{\mathrm{c}}$ & $30 \pm 2$ \\
\hline 39 & [des(tetrapeptide $\left.{ }^{\mathrm{B} 27-\mathrm{B} 30}\right)$, Glu ${ }^{\mathrm{B} 26}$ ]-insulin & $0.85 \pm 0.07(3)^{\mathrm{c}}$ & $38 \pm 4$ \\
\hline 40 & {$\left[\right.$ des(tetrapeptide $\left.{ }^{\mathrm{B} 27-\mathrm{B} 30}\right)$, Lys ${ }^{\mathrm{B} 26}$ ]-insulin } & $0.67 \pm 0.09(3)^{\mathrm{c}}$ & $48 \pm 7$ \\
\hline 41 & $\left[\right.$ des(tetrapeptide $\left.\left.{ }^{\mathrm{B} 27-\mathrm{B} 30}\right), \mathrm{Val}^{\mathrm{B} 26}\right]$-insulin & $1.74 \pm 0.22(3)^{b}$ & $24 \pm 7$ \\
\hline 42 & {$\left[\right.$ des(tetrapeptide $\left.{ }^{\mathrm{B} 27-\mathrm{B} 30}\right), \mathrm{Phe}^{\mathrm{B} 26}$ ]-insulin } & $1.01 \pm 0.22(3)^{d}$ & $27 \pm 10$ \\
\hline 43 & $\left[\right.$ des(tetrapeptide $\left.\left.{ }^{\mathrm{B} 27-\mathrm{B} 30}\right), \mathrm{His}^{\mathrm{B} 26}\right]$-insulin & $0.70 \pm 0.32(3)^{\mathrm{a}}$ & $49 \pm 27$ \\
\hline 44 & $\left[\right.$ des(tetrapeptide $\left.{ }^{\mathrm{B} 27-\mathrm{B} 30}\right)$, Pro $\left.^{\mathrm{B} 26}\right]$-insulin & $0.61 \pm 0.34(4)^{\mathrm{e}}$ & $46 \pm 26$ \\
\hline 45 & [des(tetrapeptide $\left.{ }^{\mathrm{B} 27-\mathrm{B} 30}\right)$, amide $\left.{ }^{\mathrm{B} 26}\right]$-insulin & $0.54 \pm 0.11(3)^{\mathrm{a}}$ & $63 \pm 38$ \\
\hline 46 & {$\left[\right.$ des(tetrapeptide $\left.{ }^{\mathrm{B} 27-\mathrm{B} 30}\right), \mathrm{Phe}^{\mathrm{B} 26}$, amide $^{\mathrm{B} 26}$ ]-insulin } & $0.72 \pm 0.19(3)^{\mathrm{f}}$ & $38 \pm 10$ \\
\hline 47 & $\left[\right.$ des(tetrapeptide $\left.{ }^{\mathrm{B} 27-\mathrm{B} 30}\right)$, Pro $\left.^{\mathrm{B} 26}\right]-$ amide $^{\mathrm{B} 26}$ & $0.20 \pm 0.05(4)^{g}$ & $215 \pm 54$ \\
\hline 48 & [des(tetrapeptide ${ }^{\mathrm{B} 27-\mathrm{B} 30}$ ), pTyr ${ }^{\mathrm{B} 26}$, amide $^{\mathrm{B} 26}$ ]-insulin & $0.11 \pm 0.01(3)^{\mathrm{h}}$ & $245 \pm 59$ \\
\hline
\end{tabular}


Table S13. Receptor-binding affinities of human insulin, analogs for human IR-B in membranes of transfected mouse fibroblasts. The $K_{\mathrm{d}}$ values of human insulin and analogs were determined in eight independent series of measurements indexed with a-h. The individual $K_{\mathrm{d}}$ values of analogs in the column are relative to a corresponding $K_{d}$ value of native insulin (e.g. a to a, etc.). Relative binding affinity S.D. values of analogs were calculated as S.D. $=K_{\mathrm{d}}$ of the native hormone $/ K_{\mathrm{d}}$ of analog $\mathrm{x} 100 \mathrm{x} \sqrt{ }\left[\left(\text { S.D. native/ } K_{\mathrm{d}} \text { native }\right)^{2}+\left(\right.\right.$ S.D. analog $/ K_{\mathrm{d}}$ analog) $\left.{ }^{2}\right]$.

\begin{tabular}{|c|c|c|c|}
\hline & Analog & $\begin{array}{c}K_{\mathrm{d}} \pm \text { S.D. } \\
\text { for IR-B } \\
{[\mathrm{nM}](\mathrm{n})}\end{array}$ & $\begin{array}{c}\text { Relative } \\
\text { binding affinity } \\
\text { (in \%) for IR-B }\end{array}$ \\
\hline & \multirow[t]{8}{*}{ human insulin } & $0.35 \pm 0.11(3)^{\mathrm{a}}$ & $100 \pm 31$ \\
\hline & & $0.38 \pm 0.16(3)^{b}$ & $100 \pm 42$ \\
\hline & & $0.35 \pm 0.02(3)^{\mathrm{c}}$ & $100 \pm 6$ \\
\hline & & $0.33 \pm 0.15(3)^{d}$ & $100 \pm 45$ \\
\hline & & $0.38 \pm 0.10(4)^{\mathrm{e}}$ & $100 \pm 26$ \\
\hline & & $0.37 \pm 0.03(3)^{f}$ & $100 \pm 8$ \\
\hline & & $0.67 \pm 0.17(4)^{g}$ & $100 \pm 25$ \\
\hline & & $0.32 \pm 0.12(5)^{\mathrm{h}}$ & $100 \pm 38$ \\
\hline 35 & [des(tetrapeptide $\left.{ }^{\mathrm{B} 27-\mathrm{B} 30}\right)$ ]-insulin & $1.13 \pm 0.21(3)^{\mathrm{a}}$ & $31 \pm 11$ \\
\hline 36 & [des(tetrapeptide $\left.{ }^{\mathrm{B} 27-\mathrm{B} 30}\right), \mathrm{Gly}^{\mathrm{B} 26}$ ]-insulin & $0.09 \pm 0.01(3)^{b}$ & $422 \pm 184$ \\
\hline 37 & $\left[\right.$ des(tetrapeptide $\left.\left.\left.{ }^{\mathrm{B} 27-\mathrm{B} 30}\right), \mathrm{Ser}^{\mathrm{B} 26}\right)\right]$-insulin & $0.11 \pm 0.04(3)^{\mathrm{c}}$ & $318 \pm 117$ \\
\hline 38 & [des(tetrapeptide $\left.\left.{ }^{\mathrm{B} 27-\mathrm{B} 30}\right), \mathrm{Thr}^{\mathrm{B} 26}\right]$-insulin & $1.11 \pm 0.08(3)^{\mathrm{c}}$ & $32 \pm 3$ \\
\hline 39 & $\left[\right.$ des(tetrapeptide $\left.\left.{ }^{\mathrm{B} 27-\mathrm{B} 30}\right), \mathrm{Glu}^{\mathrm{B} 26}\right]$-insulin & $0.69 \pm 0.07(3)^{\mathrm{c}}$ & $51 \pm 6$ \\
\hline 40 & {$\left[\right.$ des(tetrapeptide $\left.{ }^{\mathrm{B} 27-\mathrm{B} 30}\right)$, Lys $^{\mathrm{B} 26}$ ]-insulin } & $0.52 \pm 0.03(3)^{\mathrm{c}}$ & $67 \pm 5$ \\
\hline 41 & [des(tetrapeptide $\left.{ }^{\mathrm{B} 27-\mathrm{B} 30}\right), \mathrm{Val}^{\mathrm{B} 26}$ ]-insulin & $1.11 \pm 0.48(3)^{b}$ & $34 \pm 21$ \\
\hline 42 & [des(tetrapeptide $\left.{ }^{\mathrm{B} 27-\mathrm{B} 30}\right), \mathrm{Phe}^{\mathrm{B} 26}$ ]-insulin & $0.32 \pm 0.07(3)^{d}$ & $103 \pm 52$ \\
\hline 43 & [des(tetrapeptide $\left.{ }^{\mathrm{B} 27-\mathrm{B} 30}\right)$, His $^{\mathrm{B} 26}$ ]-insulin & $0.39 \pm 0.14(3)^{\mathrm{a}}$ & $90 \pm 43$ \\
\hline 44 & [des(tetrapeptide $\left.{ }^{\mathrm{B} 27-\mathrm{B} 30}\right)$, Pro $^{\mathrm{B} 26}$ ]-insulin & $0.27 \pm 0.08(3)^{\mathrm{e}}$ & $141 \pm 56$ \\
\hline 45 & [des(tetrapeptide $\left.{ }^{\mathrm{B} 27-\mathrm{B} 30}\right)$, amide ${ }^{\mathrm{B} 26}$ ]-insulin & $0.30 \pm 0.14(3)^{\mathrm{a}}$ & $117 \pm 66$ \\
\hline 46 & {$\left[\right.$ des(tetrapeptide $\left.{ }^{\mathrm{B} 27-\mathrm{B} 30}\right), \mathrm{Phe}^{\mathrm{B} 26}$ amide $^{\mathrm{B} 26}$ ]-insulin } & $0.65 \pm 0.07(3)^{\mathrm{f}}$ & $57 \pm 8$ \\
\hline 47 & $\left[\right.$ des(tetrapeptide $\left.{ }^{\mathrm{B} 27-\mathrm{B} 30}\right)$, Pro $\left.^{\mathrm{B} 26}\right]$-amide $^{\mathrm{B} 26}$ & $0.13 \pm 0.01(3)^{\mathrm{g}}$ & $515 \pm 137$ \\
\hline 48 & $\left[\right.$ des(tetrapeptide $\left.{ }^{\mathrm{B} 27-\mathrm{B} 30}\right), \mathrm{pTyr}^{\mathrm{B} 26}$ amide $\left.^{\mathrm{B} 26}\right]$ - insulin & $0.12 \pm 0.02(3)^{\mathrm{h}}$ & $267 \pm 110$ \\
\hline
\end{tabular}



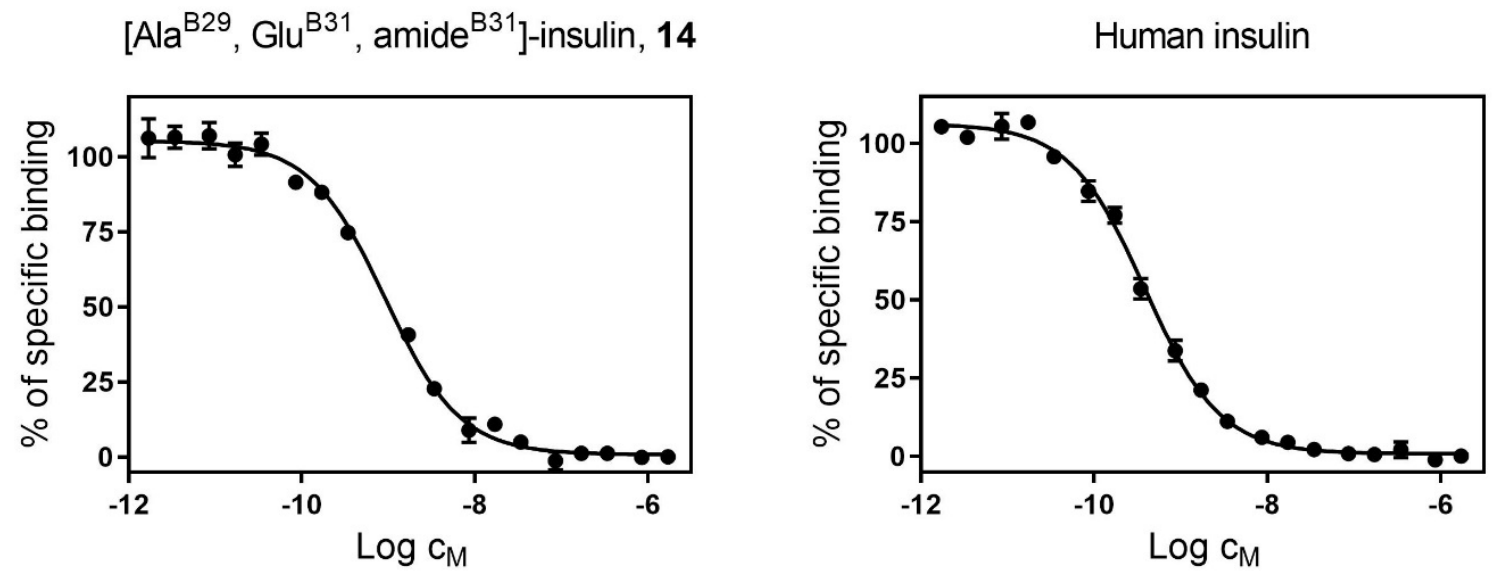

Figure S50. Representative binding curves of analog 14 (upper panel) and human insulin (lower panel) on human IR-A receptor in IM-9 lymphocytes.

\section{Representative binding curves of analog 14 and human insulin on human IR-B receptor}

$\left[\mathrm{Ala}^{\mathrm{B} 29}, \mathrm{Glu}^{\mathrm{B} 31}\right.$, amide $\left.^{\mathrm{B} 31}\right]$-insulin, 14

Human insulin
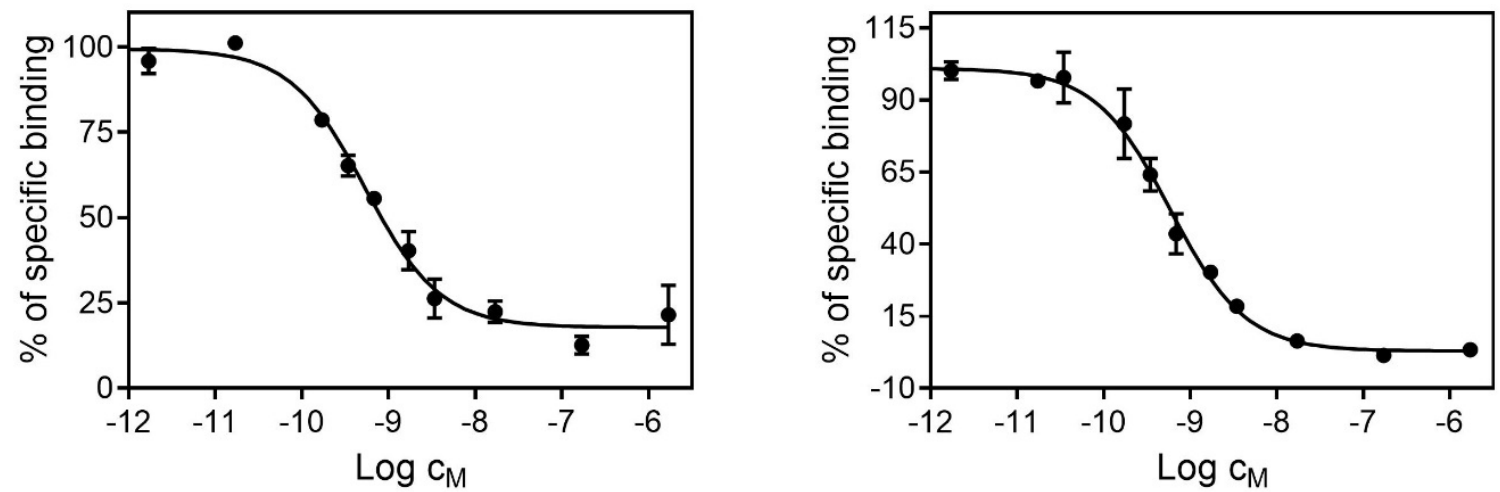

Figure S51. Representative binding curves of analog 14 (upper panel) and human insulin (lower panel) on human IR-B receptor in transfected mouse fibroblasts. 
Figure S52 shows a comparison of abilities of human insulin and analog 14 (panel A) or analog 30 (panel B) to stimulate autophosphorylation of IR-A and IR-B.
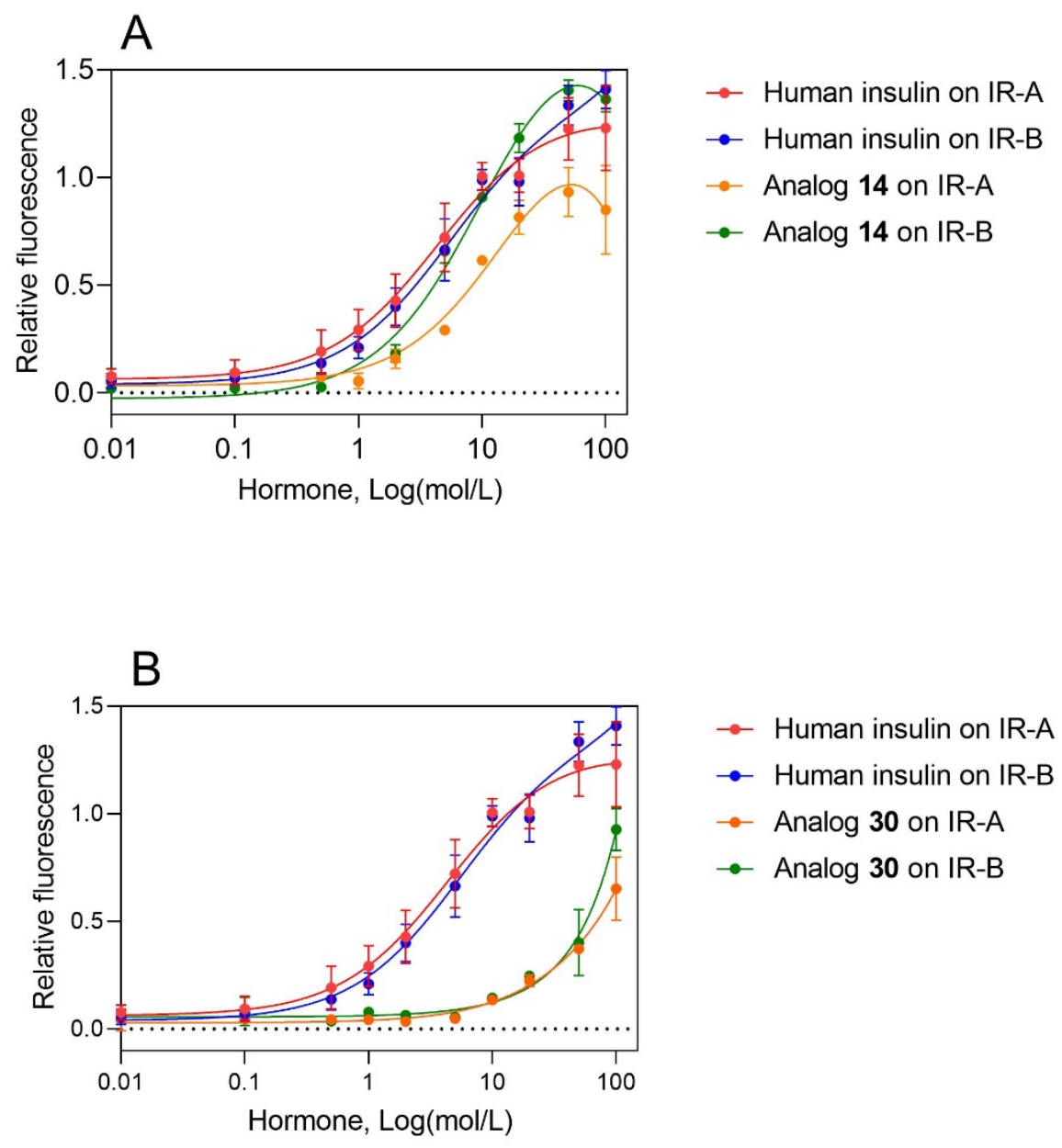

Figure S52. Abilities of human insulin and analog 14 (panel A) and human insulin and analog 30 (panel B) to stimulate autophosphorylation of IR-A and IR-B receptors. 